\title{
LA RIPARTIZIONE DI ALCUNE ZONE DELLA SUDDIVI- SIONE DI HAYFORD PER LA VALUTAZIONE DELLE RI- DUZIONI ISOSTATICHE ED ESPRESSIONI IMPIEGATE PER IL CALCOLO DEI CORRISPONDENTI NUMERI DI CASSINIS
}

\author{
Silvio Ballarin
}

\section{1. - Premessa.}

Nella riunione della Sottocommissione per la gravimetria tenutasi a Roma nel febbraio del 1952, la Commissione geodetica italiana, su proposta del prof. Cassinis, Presidente della Commissione, decideva di affidare ad un numero ristretto dei suoi membri l'incarico di formulare le modalita da tenersi nel calcolo delle riduzioni delle misure di gravita progettate dalla stessa Commissione per la costruzione della carta gravimetrica d'Itulia.

I lavori della Commissione ristretta, presieduta dal prof. Dore e composta dai professori Solaini, Morelli, rappresentante l'Istituto Nazionale di Geofisica, e dallo scrivente, ebbero termine nel giugno dello stesso anno e le proposte da essa formulate tenendo nella dovuta considerazione i risultati delle più recenti ricerche intraprese sull'argromento dal dott. Cunietti e dal prof. Morelli, vennero in linea di massima approvate dalla Commissione geodetica nell'Assemblea generale tenutasi a Milano al principio dell'anno scorso.

Fra le proposte avanzate e accolte, una riguardava in particolare la ripartizione in sottozone, nel computo delle sole riduzioni isostatiche, delle zone notevolmente ampie: $L, M, N, O_{1}$ e della riunione in un unica zona delle zone prossime alla stazione.

In seguito a tale decisione, la Presidenza della Commissione geodetica deliberava di affidarmi l'incarico di stabilire dapprima la ripartizione più conveniente di quelle quattro zone, per poi passare al calcolo dei numeri di Cassinis, che interessano la valutazione delle sole riduzioni isostatiche, relativi alla nuova suddivisione.

Nel presente lavoro viene data relazione dei criteri che hanno presieduto alla ripartizione, e dei procedimenti tenuti nel calcolo dei numeri di Cassinis. 


\section{2. - Limiti deLLE SOTTOZone CiIf SUDdividono LE ZONE $L, M, N, O_{1}$.}

1. - Nelle zone $L, M, N, O_{1}$ in conseguenza della loro eccessiva ampiezza, le variazioni con la quota dei numeri di Cassinis relativi alle profonditì che interessano le riduzioni isostatiche, superano noterolmente $i$ valori che esse assumono in tutte le altre zone. Pertanto si è giudicato conveniente suddividere ciascuna di quelle zone in un numero opportuno di sottozone in guisa che le dette variazioni in prossimità della quota $\boldsymbol{H}_{4}=-40 \mathrm{~km}$ - media dei valori generalmente adottati per la profondità della superficie di separazione nell'ipotesi di Airy-Heiskanen — risultassero pressoché uguali tra loro e nel contempo non discoste dai valori da esse assunti nelle altre zone immediatamente vicine.

Siccome nelle zone $L, M, N, O_{1}$ ed in corrispondenza della profondità $H_{0}=-40 \mathrm{~km}$, le variazioni dei numeri di Cassinis per una variazione di $-100 \mathrm{~m}$ della quota, sono rispettivamente uguali a: $+39,+104,+79,+37$ unità di $1.10^{5}$ gal, mentre nelle zone precedenti e immediatamente seguenti non superano di molto le +20 unità, si è convenuto di dividere le zone $L$ e $O_{1}$ in due sottozone, la zona $M$ in quattro, e in tre sottozone la zona $N$.

Per soddisfare infine l'altra condizione, la quale stahilisce i limiti delle sottozone, si è seguito l'orvio procedimento che qui si riporta anticipando alcuni risultati ottenuti nella ricerca dell'espressione più conveniente da impiegare nel calcolo dei numeri di Cassinis relativi alle sottozone.

2. - I numeri $f$ di Cassinis corrispondenti ad una zona generica di quota negativa $H$ e limitata sulla sfera di raggio $R$, dai ragrgi sferici $\phi_{1}, \phi_{2}$ espressi in misura angolare, sono definiti dalla relazione $[14]$

$$
f(y)=F(x, y)-F\left(x_{1}, y\right)
$$

avendo posto:

$$
y=\frac{H}{k} \quad x=\sin \frac{\phi}{2}
$$

ed indicato con $F$ la componente verticale dell'attrazione esercitata dalla calotta di spessore $H$, di ampiezza $\emptyset$ e di densità uno la cui espressione è data dalla [12].

Per calotte non molto ampie, come quelle che comprendono le 
zone indicate da Hayford con lettere, e per spessori non eccessivamente grandi, si ha dalla [12] con sufficiente approssimazione:

$$
d F=-2 \pi k R(1-\sin \alpha) d y
$$

essendo:

$$
\operatorname{tang} \alpha=\frac{|y|}{2 x}
$$

e quindi:

$$
d f=2 \pi k R\left(\sin \alpha_{, 2}-\sin \alpha_{1}\right) d y
$$

Sicché convenendo di ripartire la zona generica in $n$ sottozone con la condizione posta in precedenza - cioè in modo che in corrispondenza di una quota assegnata le variazioni con la quota dei numeri di Cassinis risultino fra loro uguali in tutte quelle $n$ sottozone - il raggio esterno dell' $i^{\text {simal }}$ sottozona dovà soddisfare l'uguaglianza :

$$
\sin \alpha_{i}=\frac{1}{n}\left[i \sin \alpha_{12}-(n-i) \sin \alpha_{1}\right]
$$

dalla quale si ricava $\alpha_{\text {: }}$ e mediante la posizione: tang $\alpha_{\mathrm{i}}=\frac{|y|}{2 x_{\mathrm{i}}}$ if valore $\phi_{\mathrm{i}}$.

Per il valore $H_{0}=-40 \mathrm{~km}$ della profondità assegnata si ottengono, arrotondando alla decina di metri, i seguenti limiti $d=R \emptyset$ delle sottozone ai quali corrispondono i valori $x$ a fianco segnati.

Limiti delle sottozone.

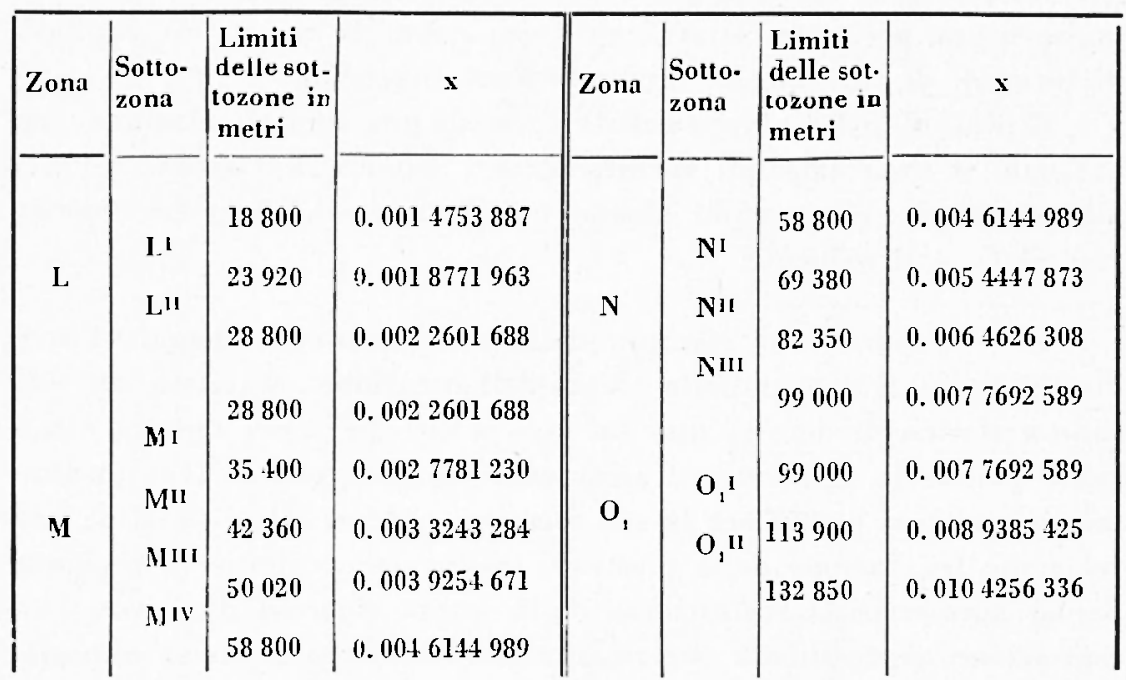


Dopo una siffatta ripartizione i valori, espressi in unità di $1.10^{-5}$ gal, delle variazioni della riduzione totale topockafico-isostatica nell'ipotesi di Airy, per le profonditi $H_{0}=-20 \mathrm{~km},-30 \mathrm{~km},-40 \mathrm{~km}$ della superficie di separazione - riduzione relativa a ciascuna delle zone che seguono la $G$ (nelle quali ordinariamente la riduzione topografica viene calcolata non più per compartimenti come nelle prime zone, ma per l'intera zona) costituite interamente da una porzione continentale di quota media $H_{1}$ uguale rispettivamente a: $0+1000 \mathrm{~m}$ $+2000 \mathrm{~m}$, o interamente ricoperte da una porzione di oceano di profonditì media $\ddot{i}$., rispettivamente uguale a: $0,-1000 \mathrm{~m},-2000 \mathrm{~m}$ - per una variazione $\lrcorner H_{1}=+190 \mathrm{~m}$ della quota del continente, o per una variazione $\Delta H_{2}=-100 \mathrm{~m}$ della profondità dell'oceano, sono contenuti nelle Tabelle I e II, tenendo presente che per le ultime tre zone le variazioni sono trascurabili.

\section{3. - Calcolo dei numeri di Cassinis con la relazione fra le com- PONENTI Verticali delle attrazioni esercitate da UNA CalotTa SFERICA E DA UNA PIASTRA.}

1. - La notevole mole di lavoro che comporta il calcolo dei numeri di Cassinis con la formula rigorosa, ha consigliato la ricerca di una relazione fra le componenti verticali delle attrazioni della calot a e della piastra le quali per le zone prossime alla stazione non differiscono eccessivamente.

L'espressione trovata, pur non essendo rappresentata da una formula chiusa, permette tuttavia, col tener conto di un numero limitato di termini, di raggiungere una sufficiente precisione.

Il procedimento tenuto nella ricerca di una siffatta relazione, con la quale si sono calcolati speditamente $i$ numeri di Cassinis relativi alle sottozone, impiegando alcune tabelle numeriche in precedenza compilate, ̀̀ il seguente.

2. - L'espressione rigorosa della componente $F$ secondo l'asse, diretto verso il centro della sfera, dell'attrazione esercitata da una calotta sferica di densità uno sul suo polo (che è poi l'intera attrazione poiché la componente orizzontale è nulla per ragioni di simmetria), poco si presta per la sua forma complessa alla ricerca di una relazione fra l'azione della piastra e quella della calotta. Si presenta perciò necessaria la sostituzione della forma rigrorosa di $F$ con una espressione approssimata che ricaveremo adottando la stessa imposta- 


\section{TABELLA I}

Variazione della riduzione topografico-isostatica nell'ipotesi di Airy e per una profondità $\mathrm{H}_{0}$ della superficie di separazione, relativa ad un continente di quota media $\mathrm{H}_{1}$ per una variazione $\Delta \mathrm{H}_{1}=-+100 \mathrm{~m}$ della quota (in unità di $1.10^{-5}$ gal).

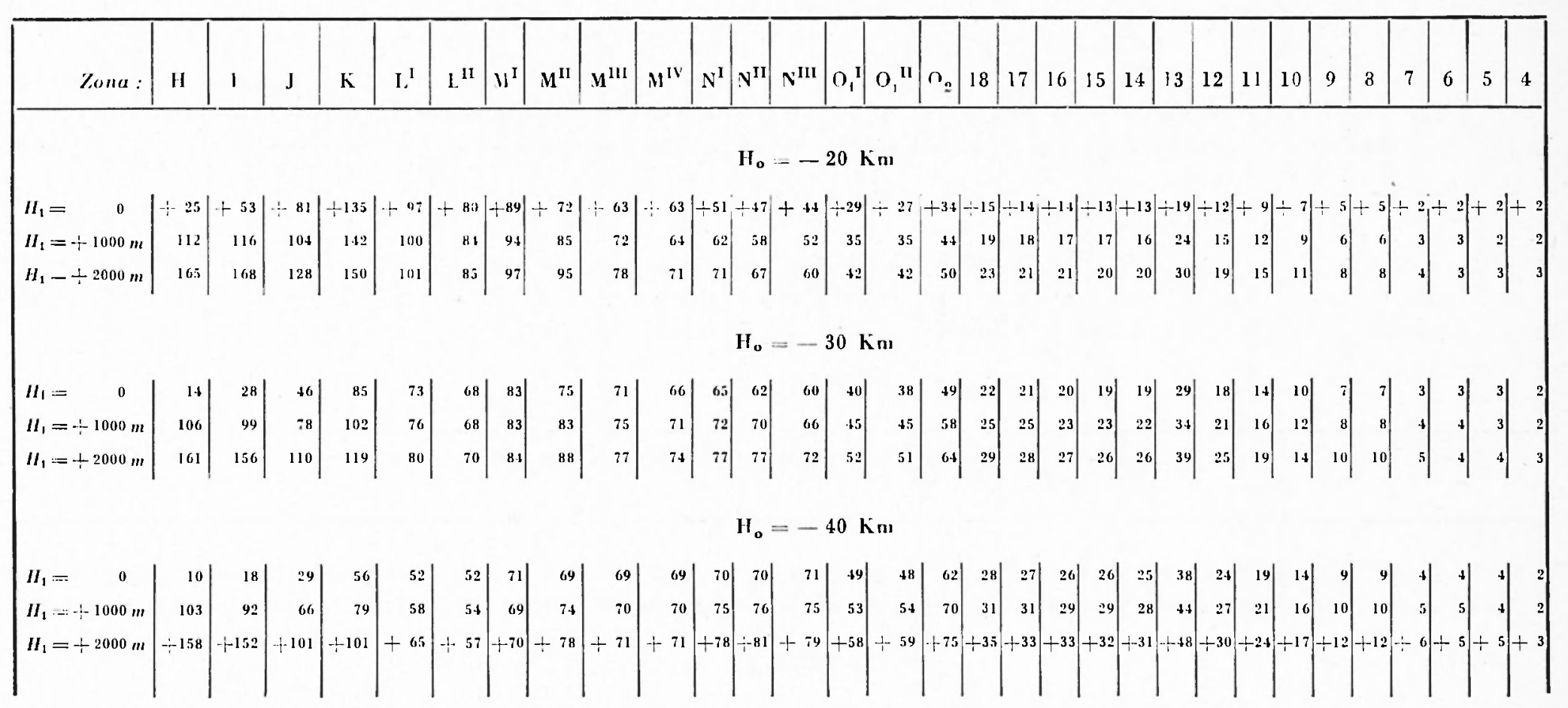




\section{Tabella II}

Variazione della riduzione topografico-isostatica nell'ipotesi di Airy e per una profondità $\mathrm{H}_{0}$ della superficie di separazione, relativa ad un oceano di profondità $\mathrm{H}_{2}$ per una variazione $\Delta \mathrm{II}_{9}=-100 \mathrm{~m}$ della profondità (in unità di $1.10^{-\overline{5}}$ gal).

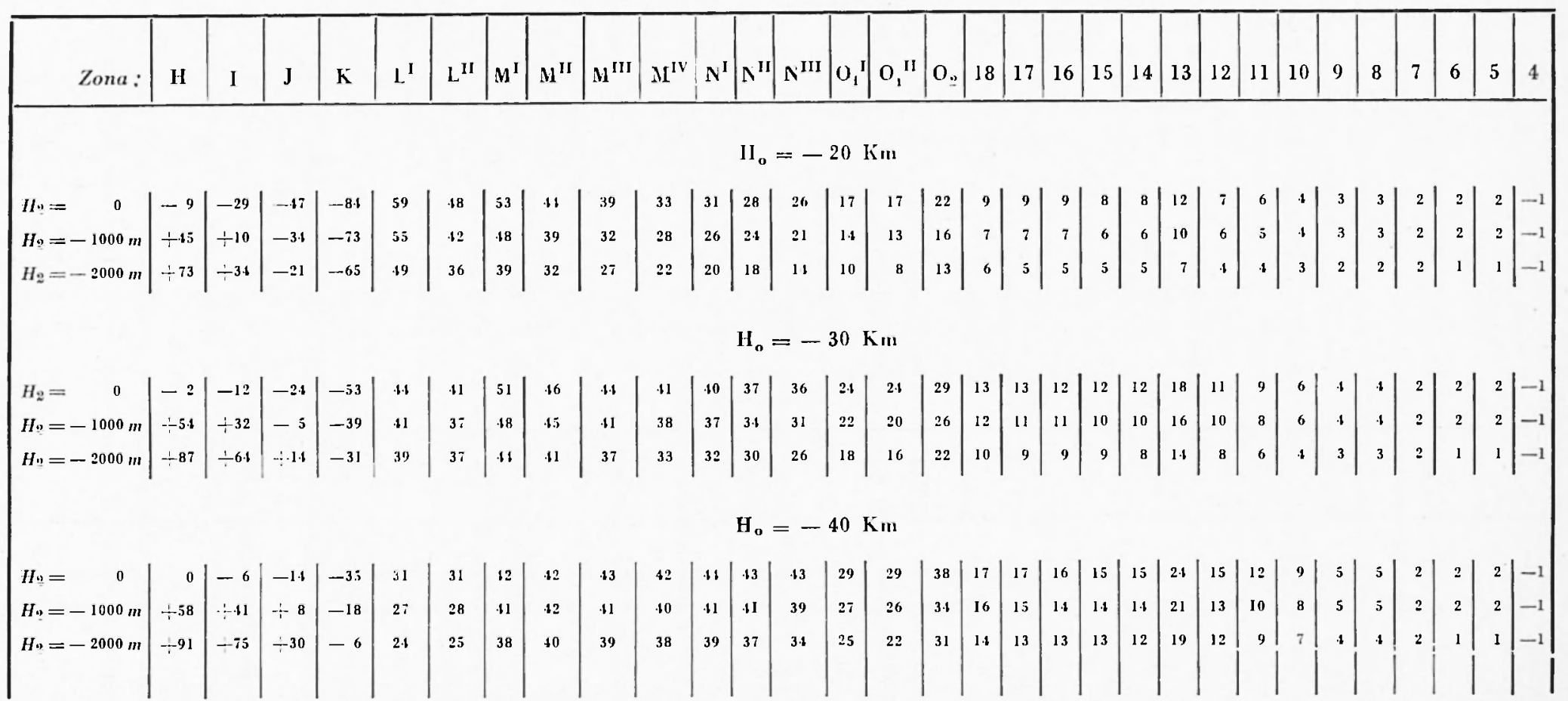


zione e le stesse notazioni impiegate dal prof. Cassinis nella determinazione dell'espressione rigorosa.

Si indichi pertanto con $S$ (fg. l) il punto potenziato che si trova sulla superficie terrestre schematizzata dalla sfera di raggio $R$ di centro $O$ e si consideri la calotta, avente il polo in $S$, limitata dalle sfere di raggio $R$ e $R+H$ e dal cono di vertice $O$, asse $O S$ e apertura $\phi$.

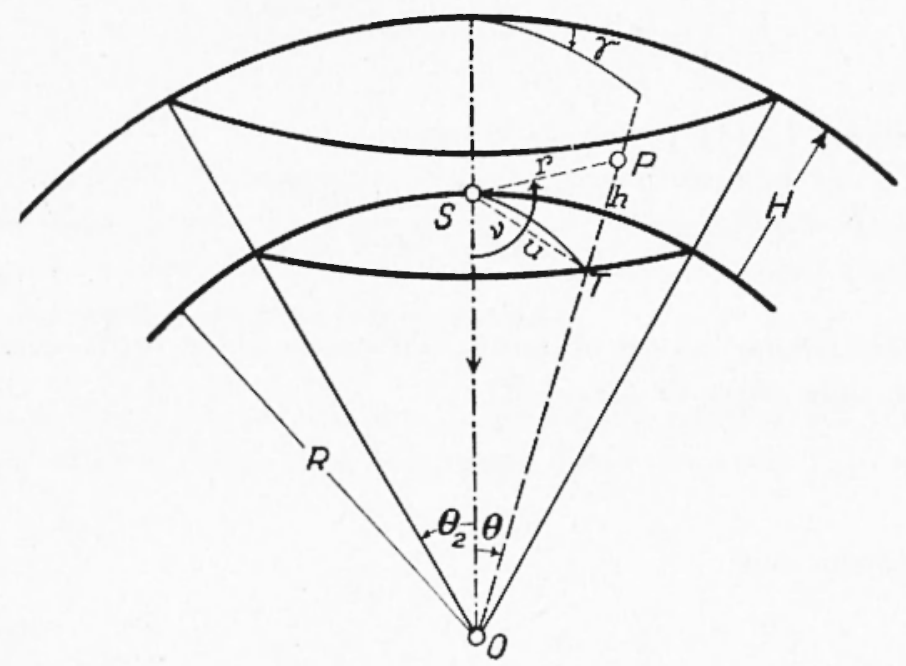

Fig. 1

La componente elementare $d F$ secondo il raggio per $S$ diretto verso il centro della sfera, dell'attrazione esercitata sull'unità di massa posta in $S$, dalla massa elementare $d m$ di densità pure unitaria del generico punto materiale $P$ della calotta, è uguale a:

$$
d F=k \frac{d m}{r^{2}} \cos \nu
$$

avendo indicato con $k$ la costante dell'attrazione, con $r$ la distanza dei due punti e con $v$ l'angolo formato dal segmento $r$ con il raggio per $S$ diretto verso il centro $O$.

Ora l'elemento di massa ha la forma:

$$
d m=(R+h)^{2} \sin \phi d \gamma d \phi . d h
$$

essendo $\gamma$ la sezione retta del diedro avente per costola l'asse del cono, ed avendo considerato come elemento di volume quello gene- 
rato dalla rotazione per un angolo $d \gamma$ attorno ad $S O$, del rettangolino $(R+h) d \phi d h$ corrispondente agli incrementi $d \emptyset$ e $d h$ dell'angolo $\emptyset$ e dell'altezza $h$ di $P$ sulla sfera terrestre; mentre l'espressione della distanza $r$ e dellangolo $\nu$ in funzione di $h$, $\varnothing$ si ricavano dal triangolo $S P O$ applicando il teorema di Carnot:

$$
\begin{gathered}
r^{-}=R^{-}+(R+h)-2 R(R+h) \cos \phi \\
\text { c s } v=\frac{R-(R+h) \cos \varphi)}{r}
\end{gathered}
$$

Pertanto la $[1]$ prende la forma:

$d F=h(R+h)^{2} \frac{R-(R+h) \cos \phi}{\left[R-(R+h)^{2}-2 R(R+h) \cos \phi\right]^{3 / 2}} \sin \phi d h d \phi d \gamma$

Introduciamo adesso al posto dell'angolo $\phi$ la corda corrispondente usulla sfera di raggio $R$ :

$$
u=2 R \sin \frac{\phi}{2}
$$

e indichiamo con:

$$
x=\sin \frac{\not}{2} \quad y=\frac{h}{R}
$$

Assumendo $x$ e $y$ come nuove variabili la [3] diventa:

$$
d F=2 \pi k R(1+y)^{2} \frac{2 x^{2}(1+y-y}{\left[y^{2}+4 x^{2}(1+y)\right]^{3 / 2}} d y d\left(x^{2}\right) d \gamma
$$

Segnando con $D$ il valore aritmetico della radice:

$$
D=\sqrt{y^{2}+4 x^{2}(1+y)}
$$

si avrà per la [2]

$$
r=R D
$$

e per le [4] [4']

$$
u=R 2 x \quad h=R y
$$

Quindi i valori $D, 2 x$, essenzialmente positivi, e $y$ rappresentano rispettivamente: la distanza $r$, la corda $u$, e l'altezza $h$ espresse in ragrgi terrestri. 
L'attrazione dell'intera calotta si ottiene integrando la [5] fra i limiti $\gamma(=0 ; 2 \pi) ; x\left(=0 ; \sin \frac{\varnothing}{2}\right), y\left(=0 ; \frac{H}{R}\right)$ mentre la componente verticale dell'attrazione esercitata da un settore di calotta si ottiene evidentemente sostituendo a $2 \pi$ l'ampiezza $\gamma$ del settore.

Eseguendo effettivamente le due prime integrazioni si ha:

$$
F=\left.2 \pi k R\right|_{0} ^{y}(1+y)^{2}\left[\frac{y+2 x^{2}}{D}\right]_{0}^{x} d y
$$

Effettuando l'integrazione rigorosa anche rispetto ad $y$ il prof. Cassinis ha ottenuto da questa l'espressione chiusa, più sopra ricordata, che ha servito al calcolo delle Tavole fondamentali per la riduzione dei valori osservati della gravità.

3. - La [7] si può mettere in forma più conveniente per il successivo sviluppo del calcolo, osservando a tale proposito l'uguaglianza:

$$
\frac{\partial}{\partial y} D=\frac{2 x^{2}+y}{D}
$$

Si ha vertanto

$$
F=2 \pi k R \int_{0}^{y}(1+y)^{z}\left[\frac{\partial}{\partial y} D\right]_{0}^{x} d y
$$

cioè

$$
\begin{aligned}
& F=2 \pi k R \int_{0}^{y}(1+y)^{2}\left(\frac{\partial}{\partial y} D-1\right) d y= \\
& \quad=2 \pi k R\left[i_{0}^{l_{0}^{y}}(1+y)^{2} \frac{\partial D}{\partial y} d y-\left(y+y^{3}+\frac{y^{3}}{3}\right)\right]
\end{aligned}
$$

ed integrando per parti:

$$
F=2-k R\left[(1+y)^{2} D-\left(y+y^{2}+\frac{y^{3}}{\overline{3}}+2 x\right)-2 \int_{0}^{y}(1+y) D d y\right]
$$


Indicando ora con $d$ il valore aritmetico della radice:

$$
d=\sqrt{y^{2}+(2 x)^{2}}
$$

e ponendo:

$$
\cos \alpha=\frac{2 x}{d}
$$

si ha

$$
D=d\left[1+\cos ^{\prime \prime} \alpha y\right]^{1 / 2}
$$

e sviluppando in serie:

$D=d\left[1+\frac{1}{2} \cos ^{\prime \prime} \alpha y-\frac{1}{8} \cos ^{4} \alpha y^{\prime \prime}+\frac{1}{16} \cos ^{6} \alpha y^{3}-\frac{5}{128} \cos ^{8} \alpha y^{4}+\ldots\right]$

Ma per le posizioni fatte:

$$
d=\frac{y}{\sin \alpha}
$$

e quindi :

$$
\begin{aligned}
D=y\left[\frac{1}{\sin \alpha}+\right. & \frac{1}{2} \operatorname{cotg} \alpha \cos \alpha y-\frac{1}{8} \operatorname{cotg} \alpha \cos ^{3} \alpha y^{2}+ \\
& \left.+\frac{1}{16} \operatorname{cotg} \alpha \operatorname{ccs} \alpha y^{3}-\frac{5}{128} \operatorname{cotg} \alpha \cos ^{7} \alpha y^{4}+\ldots \ldots \ldots\right]
\end{aligned}
$$

Cosi :

$$
\begin{aligned}
(1+y)^{2} D=\frac{1}{\sin \alpha} y & +\left[2 \frac{\sin \alpha}{\cos ^{2} \alpha}+\frac{1}{2} \sin \alpha\right] \operatorname{cotg} \alpha y^{2} \\
& +\left[\frac{\sin ^{2} \alpha}{\cos ^{3} \alpha}-\frac{1}{8} \sin ^{2} \alpha \cos \alpha+\frac{\sin ^{2} \alpha}{\cos \alpha}\right] \operatorname{cotg}^{3} \alpha y^{3} \\
& +\frac{1}{2}\left[\frac{\sin ^{3} \alpha}{\cos ^{2} \alpha}-\frac{1}{2} \sin ^{3} \alpha+\frac{1}{8} \sin ^{3} \alpha \cos ^{2} \alpha\right] \operatorname{cotg}^{4} \alpha y^{4} \\
+\frac{1}{4}\left[-\frac{1}{2} \frac{\sin ^{4} \alpha}{\cos \alpha}\right. & \left.+\frac{1}{2} \sin ^{i} \alpha \cos \alpha-\frac{5}{32} \sin ^{i} \alpha \cos ^{3} \alpha\right] \operatorname{cotg}^{5} \alpha y^{5}+\ldots . .
\end{aligned}
$$


$(1+y) I)=\frac{1}{\cos \alpha}(2 x)+\left[\frac{1}{2}+\frac{1}{\cos ^{2} \alpha}\right](2 x)^{2} \sin \alpha-$

$-\frac{1}{2}\left[\frac{1}{4}-\frac{1}{\cos ^{2} \alpha}\right](2 x)^{3} \sin ^{2} \alpha \cos \alpha+\frac{1}{8}\left[\frac{1}{2}-\frac{1}{\cos ^{2} \alpha}\right](2 x)^{4} \sin ^{3} \alpha \cos ^{2} \alpha-$

$-\frac{1}{16}\left[\frac{5}{8}-\frac{1}{\cos ^{10} \alpha}\right](2 x)^{5} \sin ^{4} \alpha \cos ^{3} \alpha+\ldots \ldots \ldots$

e poiché

$$
d y=(2 x) \frac{1}{\cos ^{2} \alpha} d \alpha
$$

ne serue:

$$
\begin{aligned}
& (1+y) D d y=\left\{\frac{1}{\cos ^{\prime} \alpha}(2 x)^{2}+\left[\frac{1}{2}+\frac{1}{\cos ^{2} \alpha}\right](2 x)^{3} \frac{\sin \alpha}{\cos \alpha^{2}}-\right. \\
& -\frac{1}{2}\left[\frac{1}{4}-\frac{1}{\cos ^{9} \alpha}\right](2 x)^{4} \frac{\sin ^{2} \alpha}{\cos \alpha}+\frac{1}{8}\left[\frac{1}{2}-\frac{1}{\cos ^{2} \alpha}\right](2 x)^{5} \sin ^{3} \alpha- \\
& \left.-\frac{1}{16}\left[\frac{5}{8}-\frac{1}{\cos ^{2} \alpha}\right](2 x)^{6} \sin ^{4} \alpha \cos \alpha+\ldots \ldots\right\} d \alpha
\end{aligned}
$$

Integrando e ponendo in evidenza, dopo la limitazione, le potenze ascendenti di $y$ si ottene:

$$
\begin{aligned}
& \int_{0}^{y}(1+y) D d y=\frac{1}{2}\left[\frac{\sin \alpha}{\cos ^{2} \alpha}+\ln \tan g\left(\frac{\pi}{4}-\frac{\alpha}{2}\right)\right] \operatorname{cotg}^{2} \alpha y^{2}+ \\
& +\frac{1}{2}\left[\frac{1}{\cos \alpha}+\frac{2}{3} \frac{1}{\cos ^{3} \alpha}-\frac{5}{3}\right] \operatorname{cotg}^{3} \alpha y^{3}+ \\
& +\frac{1}{4}\left[\frac{1}{2} \sin \alpha-\frac{3}{2} \ln \operatorname{tang}\left(\frac{\pi}{4}+\frac{\alpha}{2}\right)+\frac{\sin \alpha}{\cos ^{2} \alpha}\right] \operatorname{cotg} \alpha y^{4}-1 \\
& +\frac{1}{8}\left[\frac{7}{3}-\frac{1}{6} \sin ^{2} \alpha \cos \alpha-\frac{4}{3} \cos \alpha-\frac{1}{\cos \alpha}\right] \operatorname{cotg}^{5} \alpha y^{6}+\ldots \ldots \ldots
\end{aligned}
$$

e pertanto la $\left[9^{\prime}\right]$ prende la forma:

$$
\begin{aligned}
F=-2 \pi k R\left\{\left[1-\operatorname{lang} \frac{\alpha}{2}\right] y+\right. & {\left[\ln \operatorname{tang}\left(\frac{\pi}{4}+\frac{\alpha}{2}\right)-\right.} \\
& \left.-\operatorname{tang} \alpha \operatorname{cotg}\left(\frac{\pi}{4}-\frac{\alpha}{2}\right)-\frac{1}{2} \sin \alpha\right] \operatorname{cotg} \alpha y^{2}+
\end{aligned}
$$




$$
\begin{aligned}
& \left.+\mid \frac{9}{8} \cos \alpha-\frac{1}{8} \cos ^{3} \alpha-\frac{5}{3}+\frac{1}{3 \cos ^{2} \alpha}\left(3 \cos ^{2} \alpha-1\right)+\frac{1}{3} \operatorname{tang}^{3} \alpha\right] \operatorname{cotg}^{3} \alpha y^{3}+ \\
& +\frac{3}{4}\left[\sin \alpha+\frac{1}{4} \sin ^{3} \alpha+\frac{1}{12} \sin ^{5} \alpha-\ln \operatorname{tang}\left(\frac{\pi}{4}+\frac{\alpha}{2}\right)\right] \operatorname{cotg}^{4} \alpha y^{4}+ \\
& \left.+\frac{1}{4}\left|\frac{7}{3}-\frac{1}{2 \cos \alpha}-3 \cos \alpha+\frac{175}{96} \cos ^{3} \alpha-\frac{13}{16} \cos ^{5} \alpha+\frac{5}{32} \cos ^{2} \alpha\right| \operatorname{cotg}^{5} \alpha y^{5}+\ldots \ldots \ldots\right\}
\end{aligned}
$$

o anche ponendo:

$\Phi_{1}(\alpha)=\left[1-\tan s \frac{\alpha}{2}\right]$

$\Phi_{\varphi}(\alpha)=\left[\frac{1}{2} \sin \alpha+\operatorname{tang} \alpha \operatorname{colg}\left(\frac{\pi}{4}+\frac{\alpha}{2}\right)-\ln \operatorname{tang}\left(\frac{\pi}{4}+\frac{\alpha}{2}\right)\right] \operatorname{cotg}^{2} \alpha$

$\Psi_{3}(x)=\left[\frac{1}{3} \frac{1}{\cos ^{3} \alpha}-\frac{1}{\cos \alpha}+\frac{5}{3}-\frac{9}{8} \cos \alpha+\frac{1}{8} \cos ^{3} \alpha-\frac{1}{3} \operatorname{tang}^{3} \alpha\right] \operatorname{cotg}^{3} \alpha$

$\Phi_{4}(\alpha)=\frac{3}{4}\left[\ln \operatorname{tang}\left(\frac{\pi}{4}+\frac{\alpha}{2}\right)-\sin \alpha-\frac{1}{4} \sin ^{3} \alpha-\frac{1}{12} \sin ^{5} \alpha\right] \operatorname{cotg}^{4} \alpha$

$\Phi_{5}(\alpha)=\frac{1}{4}\left[\frac{1}{2 \cos \alpha}-\frac{7}{3}+3 \cos \alpha-\frac{175}{96} \cos ^{3} \alpha+\frac{13}{16} \cos ^{5} \alpha-\frac{5}{32} \cos ^{2} \alpha\right] \operatorname{cotg}^{5} \alpha$

ne segue:

$F=-2 \pi k R ; \Phi_{1}(\alpha) y-\left[\Phi_{0}(\alpha) y^{\alpha}+\Phi_{3}(\alpha) y^{3}+\Phi_{4}(\alpha) y^{4}+\Phi_{5}(\alpha) y^{5}+\ldots \ldots \ldots\right]$;

Convenendo di assumere lo spessore $H$ della calotta come una quota riferita alla superficie terrestre di ragrio $R$, sulla quale griace il punto potenziato $S$, la formola trovata vale ancora quando il punto potenziato anziché trovarsi sulla superficie interna della calotta giace su quella esterna, nel qual caso $R-H$ è il raggio della sfera che limita internamente la calotta stessa.

Per le posizioni fatte con le 110, l'angolo $\alpha$ si ricava dalla relazione

$$
\operatorname{tang} \alpha=\frac{|y|}{2 x}
$$

dove per la $\left[f^{\prime}\right]$ :

$$
y=\frac{H}{R}
$$


attribuendo ad $H$ il segno positivo o quello negativo secondo che la superficie terrestre, sulla quale si trova il punto potenziato, limita internamente o esternamente la calotta.

L'espressione ricavata mette in relazione l'azione della calotta con quella esercitata dalla piastra, rappresentando il primo termine:

$$
-2 \pi k R\left(1-\operatorname{tang} \frac{\alpha}{2}\right) y=-2 \pi k H\left(1-\operatorname{tang} \frac{\alpha}{2}\right)
$$

l'attrazione della piastra cilindrica avente per raggio la corda, relativa alla superficie terrestre, della calotta e per spessore l'altezza di questa.

Le funzioni $\Phi_{:,}, \Phi_{3}, \ldots \ldots$... si lasciano facilmene taluulare prendendo come argomento: $\operatorname{tang} \alpha$; nelle Tabelle III sono riportati $i$ valori di $\Phi$. ., e $\Phi_{3}$ corrispondenti agli argomenti compresi fra 0.1 e 20. L'approssimazione tenuta nel calcolo di queste tabelle è tale cbe per valori di $H$ che non superano in valore assoluto $70 \mathrm{~km}$, l'approssimazione della [12] è dell'ordine di $0.410^{-5}$ gal.

Il calcolo di: $\operatorname{tang} \frac{\alpha}{2}$ viene facilitato adoperando le Tables a 3 decimales des valeurs naturalles des sinus, cosinus e tangentes dans lo systeme decimal, calcolate da Brandicourt sotto la direzione di Roussilhe ed edite dalla Sezione di geodesia dell'Unione geodetica e geofisica internazionale (Pubblicazione speciale n. 1 - Parigi 1925).

In quanto ai coefficienti di $y^{4}, y^{\prime \prime}$ essi crescono in valore assoluto col diminuire di $\alpha$ e per piccoli valori di $\alpha$ risultano dell'ordine:

$$
\Phi_{i}=\frac{1}{16} \frac{2 x}{y} \quad \mid \Phi_{z} !<\frac{1}{128} \frac{2 x}{y}
$$

Quindi mentre il contributo del termine di $\Phi_{\bar{\pi}}$ nella formazione della [12] è sempre inferiore in valore assoluto a $0.0610^{\text {sal }}$ ger valori di $H$ aritmeticamente non superiori a $125 \mathrm{~km}$, il contributo di $\Phi_{4}$ ¿̀ trascuralile, nell'ordine di approssimazione tenuto nel calcolo delle precedenti tabelle, quando $H$ è inferiore in valore assoluto a $25 \mathrm{~km}$; inoltre per $\boldsymbol{H}=70 \mathrm{~km}$ tale contributo non supera $0.210^{-7}$ gal quando $x$ è inferiore a 0.04 ciò̀ per distanze $d$ non eccedenti $\mathrm{i}$ $500 \mathrm{~km}$.

Anche il contributo del termine $\bar{\Phi}_{3} \grave{e}$ trascurabile quando $|H|<0.6 \mathrm{~km} ; \dot{e}$ pure trascurabile per $H<5 \mathrm{~km}$ quando $d$ non supera $170 \mathrm{~km}$. 
TABella III. - Valori delle espressioni: $\Phi_{2}, \Phi_{3}$.

\begin{tabular}{|c|c|c|c|c|c|}
\hline $\operatorname{tang} \alpha$ & $\begin{array}{cc} & \Delta \\
D_{2} & \text { differenza } \\
\text { per } 0.0001 \\
\end{array}$ & $\Phi_{3}$ & $\operatorname{tang} \alpha$ & $\Phi_{2} \begin{array}{c}\Delta \\
\text { differenzal } \\
\text { per } 0.0001\end{array}$ & $\Phi_{3}$ \\
\hline 0.1000 & $+4.0+17$ & +3.42 & 0.1135 & +3.4526 & +2.98 \\
\hline 0.1005 & 4. 0170 & 3. 40 & 0.1140 & 3. 4335 & 2. 96 \\
\hline 0.1010 & $3.9926+8.8$ & 3. 39 & $0.11+5$ & $3.4145^{38.0}$ & 2. 95 \\
\hline 0.1015 & $3.9684 \quad+8.4$ & 3.37 & 0.1150 & $3.3957 \quad 37.6$ & 2. 94 \\
\hline 0.1020 & 3. $94+4$ & 3. 35 & 0.1155 & 3.3771 & 2. 92 \\
\hline 0.1025 & 3. $9207 \quad 46.8$ & 3. 33 & 0.1160 & $\begin{array}{ll}3.3587 \quad 36.6\end{array}$ & 2. 91 \\
\hline 0.1030 & $\begin{array}{l}3.8973 \quad 46.6 \\
\end{array}$ & 3. 31 & 0.1165 & $3.3404 \quad 36.4$ & 2. 90 \\
\hline 0.1035 & 3. $8740 \quad 46.0$ & 3. 30 & 0.1170 & $\begin{array}{l}3.3222 \quad 36.0\end{array}$ & 2. 88 \\
\hline 0.1040 & $3.8510 \quad+5.6$ & 3. 28 & 0.1175 & $3.30+2 \quad 35.6$ & 2. 87 \\
\hline $0.10+5$ & 3. $8282+5.2$ & 3. 26 & 0.1180 & 3. $2864 \quad 35.2$ & 2. 85 \\
\hline 0.1050 & $\begin{array}{l}3.8056 \quad+4.6 \\
\end{array}$ & 3. 25 & 0.1185 & $\begin{array}{ll}3.2688 & \\
& \end{array} 5.0$ & 2. 84 \\
\hline 0.1055 & $\begin{array}{l}3.7833 \quad 4.4 \\
\end{array}$ & 3. 23 & 0.1190 & 3. $2513 \quad 34.8$ & 2.83 \\
\hline 0.1060 & $\begin{array}{ll}3.7611 \quad 43.8\end{array}$ & 3. 21 & 0.1195 & 3. $2339 \quad 34.6$ & 2. 81 \\
\hline 0.1065 & $\begin{array}{l}3.7392+3.4 \\
\end{array}$ & 3. 19 & 0.1200 & 3. $2166 \quad 34.2$ & 2. 80 \\
\hline 0.1070 & $\begin{array}{l}3.7175+3.2\end{array}$ & 3. 18 & 0.1205 & $\begin{array}{ll}3.1995 & \\
\text { 33. }\end{array}$ & ?. 79 \\
\hline 0.1075 & $3.6959+2.6$ & 3. 16 & 0.1210 & $\begin{array}{ll}3.1826 & \\
& 33.6\end{array}$ & 2. 77 \\
\hline 0. 1080 & $3.6746+2.2$ & 3. 15 & 0.1215 & 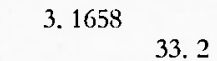 & 2. 76 \\
\hline 0.1085 & $3.6535+1.8$ & 3. 13 & 0.1220 & 3. $1+92 \quad 33.0$ & 2. 75 \\
\hline 0.1090 & $\begin{array}{ll}3.6326 & \\
& \end{array}$ & 3. 11 & 0.1225 & 3. 1327 & 2. 74 \\
\hline 0.1095 & 3. $6118+1.0$ & 3. 10 & 0.1230 & $\begin{array}{l}3.1163 \quad 32.6 \\
\end{array}$ & 2. 72 \\
\hline 0.1100 & 3. 5913 & 3. 08 & 0.1235 & $\begin{array}{l}3.1000 \\
32.2\end{array}$ & 2. 71 \\
\hline 0.1105 & 3.5709 & 3. 07 & 0.1240 & $\begin{array}{l}3.0839 \\
\quad 32.0\end{array}$ & 2. 70 \\
\hline 0.1110 & 3. 5507 & 3. 05 & 0.1245 & $\begin{array}{l}3.0679 \quad 31.6 \\
\end{array}$ & 2. 69 \\
\hline 0.1115 & 3. 5307 & 3. 04 & 0.1250 & $\begin{array}{ll}3.0521 & \\
& 31.6\end{array}$ & 2. 68 \\
\hline 0.1120 & 3. $5109 \quad 39.2$ & 3.02 & 0.1255 & 3. 0363 & 2. 66 \\
\hline 0.1125 & 3. $+913 \quad 390$ & 3. 01 & 0.1260 & 3.0207 & 2. 65 \\
\hline 0.1130 & $\begin{array}{r}+3.4718 \\
--38.4\end{array}$ & +2.99 & 0.1265 & $+3.0053-30.8$ & -2.64 \\
\hline
\end{tabular}


Contmina Tab. III.

\begin{tabular}{|c|c|c|c|c|c|}
\hline $\operatorname{tang} \alpha$ & $\begin{array}{cc} & \Delta \\
\Phi_{2} & \text { differenza } \\
\text { per } 0.0001\end{array}$ & $\Phi_{3}$ & $\operatorname{tang} \alpha$ & 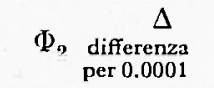 & $\Phi_{3}$ \\
\hline 0.1270 & +2.9899 & +2.63 & 0.1405 & +2.6172 & +2.35 \\
\hline 0.1275 & 2. 9747 & 2. 62 & 0.1410 & 2. 6048 & 2. 34 \\
\hline 0. 1280 & $2.9596 \quad 30.2$ & 2. 60 & 0.1415 & $2.5925 \quad 24.6$ & 2. 33 \\
\hline 0.1285 & 2. $9446 \quad 30.0$ & 2. 59 & 0.1420 & 2. 5803 & 2. 32 \\
\hline 0.1290 & 2. $9297 \quad 29.8$ & 2. 58 & 0.1425 & 2. $5681 \quad 24.4$ & 2. 31 \\
\hline 0.1295 & 2. $91+9 \quad 29.6$ & 2. 57 & 0.1430 & 2. $5561 \quad 24.0$ & 2. 30 \\
\hline 0.1300 & 2. 9003 & 2. 56 & 0.1435 & 2. $5441 \quad 24.0$ & 2. 29 \\
\hline 0.1305 & 2. 8858 & 2. 55 & 0.1440 & 2. $5322 \quad 23.8$ & 2. 28 \\
\hline 0.1310 & $2.871+\quad 28.6$ & 2. 54 & 0.1445 & 2. 5204 & 2. 27 \\
\hline 0.1315 & $2.8571 \quad 28.4$ & 2. 53 & 0.1450 & 2. 5087 & 2. 26 \\
\hline 0.1320 & $\begin{array}{l}2.8429 \\
\end{array}$ & 2. 52 & 0.1455 & 2. 4970 & 2.25 \\
\hline 0.1325 & 2. 8288 & 2. 51 & 0.1460 & $\begin{array}{ll}2.4855 & \\
& 23.0\end{array}$ & 2. 25 \\
\hline 0.1330 & 2. 8148 & 2. 49 & 0.1465 & $\begin{array}{ll}2.4740 & \\
& 22.8\end{array}$ & 2. 24 \\
\hline 0.1335 & 2. 8009 & 2. 48 & 0.1470 & 2. 4626 & 2. 23 \\
\hline 0.1340 & 27. 2 & 2. 47 & 0.1475 & 22.6 & 2. 22 \\
\hline 0.1345 & 2. 7735 & 2. 46 & 0.1480 & 2. 4400 & 2. 21 \\
\hline 0.1350 & 2. 7599 & 2. 45 & 0.1485 & 22.2 & 2. 20 \\
\hline 0.1355 & 2. 7464 & 2. 44 & 0.1490 & 2. 4177 & 2. 19 \\
\hline 0.1360 & 2. 7331 & 2. 43 & 0.1495 & 2. 4067 & 2. 19 \\
\hline 0.1365 & 2. 7199 & 2. 42 & 0.1500 & 2. 3958 & 2. 18 \\
\hline 0.1370 & 2. 7067 & 2. 41 & 0.1505 & 2. 3849 & 2. 17 \\
\hline 0.1375 & 2. 6936 & 2. 40 & 0.1510 & 21.4 & 2. 16 \\
\hline 0.1380 & 2. 6807 & 2. 39 & 0.1515 & 2. 3634 & 2. 15 \\
\hline 0.1385 & 2. $667 \mathrm{~s}$ & 2. 38 & 0.1520 & 2. 3528 & 2. 14 \\
\hline 0.1390 & 2. 6550 & 2. 37 & 0.1525 & 2. 3422 & 2. 14 \\
\hline 0.1395 & 2. $6+23$ & 2. 36 & 0.1530 & 2. 3317 & 2. 13 \\
\hline 0.1400 & $\begin{array}{r}+2.6297 \\
-25.0\end{array}$ & $\div 2.35$ & 0.1535 & $+2.3213-20.8$ & +2.12 \\
\hline
\end{tabular}


Continua Tab. III.

\begin{tabular}{|c|c|c|c|c|c|}
\hline $\operatorname{tang} \alpha$ & $\Phi_{9} \underset{\begin{array}{c}\text { differenza } \\
\text { per } 0.0001\end{array}}{\Delta}$ & $\Phi_{3}$ & $\operatorname{tang} \alpha$ & $\begin{array}{c}\Delta \\
\Phi_{2} \\
\begin{array}{c}\text { differenza } \\
\text { per } 0.0001\end{array}\end{array}$ & $\Phi_{3}$ \\
\hline 0.1540 & $+2.3109-20.6$ & +2.11 & 0.1675 & +2.0548 & +1.92 \\
\hline 0.1545 & 2. $3006 \quad 20.4$ & 2. 10 & 0.1680 & 2. 0461 & 1. 91 \\
\hline 0.1550 & 2. 2904 & 210 & 0.1685 & 2. 0375 & 1.90 \\
\hline 0.1555 & 2. 2802 & 2. 09 & 0.1690 & 2. 0289 & 1.90 \\
\hline 0.1560 & 2. 2701 & 2.08 & 0.1695 & 2. 0204 & 1. 89 \\
\hline 0.1565 & 2. 2601 & 2.07 & 0.1700 & 2. 0120 & 1. 88 \\
\hline 0.1570 & 2. 2501 & 2.07 & 0.1705 & 2. 0036 & 1. 88 \\
\hline 0.1575 & 2. 2402 & 2. 06 & 0.1710 & 1. 9952 & 1. 87 \\
\hline 0.1580 & 2. 2304 & 2. 05 & 0.1715 & 1.9869 & 1. 86 \\
\hline 0.1585 & 2. 2206 & 2. 05 & 0.1720 & 1.9786 & 1.86 \\
\hline 0.1590 & 2. 2109 & 2.04 & 0.1725 & 1.9704 & 1.85 \\
\hline 0.1595 & 2. 2012 & 2. 03 & 0.1730 & 1. 9622 & 1. 85 \\
\hline 0.1600 & 19.2 & 2. 02 & 0.1735 & 1. 9541 & 1.84 \\
\hline 0.1605 & 2. 1821 & 2. 01 & 0.1740 & 1. 9460 & 1. 83 \\
\hline 0.1610 & 2. 1726 & 2. 01 & 0.1745 & 1. 9380 & 1. 83 \\
\hline 0.1615 & 2. 1632 & 200 & 0.1750 & 1. 9300 & 1. 82 \\
\hline 0.1620 & 2. 1539 & 1. 99 & 0. 1755 & 1. 9221 & 1. 81 \\
\hline 0. 1625 & 2. 1446 & 1. 99 & 0.1760 & 1. 9142 & 1.81 \\
\hline 0.1630 & 2. 1354 & 1. 98 & 0.1765 & 1.9063 & 1. 80 \\
\hline 0.1635 & 2. $1262 \quad 18.4$ & 1. 97 & 0.1770 & 1. $8985 \quad 15.6$ & 1. 80 \\
\hline 0.1640 & 2. 1171 & 1. 96 & 0.1775 & 1.8908 & 1.79 \\
\hline 0.1645 & 2. 1080 & 1. 96 & 0.1780 & 1. 8831 & 1. 79 \\
\hline 0.1650 & 2.0990 & 1.95 & 0.1785 & 1. 8754 & 1. 78 \\
\hline 0.1655 & 2. 0901 & 1.94 & 0.1790 & 1.8678 & 1. 77 \\
\hline 0.1660 & 2. 0812 & 1.94 & 0.1795 & 1.8602 & 1. 77 \\
\hline 0.1665 & 2. $0723 \quad 17.8$ & 1.93 & 0.1800 & 1.8527 & 1. 76 \\
\hline 0.1670 & $\begin{array}{r}17.6 \\
+2.0635-17.4\end{array}$ & +1.92 & 0.1805 & $\begin{array}{rr} & 15.0 \\
+1.8452 & -14.8\end{array}$ & +1.76 \\
\hline
\end{tabular}


Contima Tab. III

\begin{tabular}{|c|c|c|c|c|c|}
\hline $\operatorname{tang} \alpha$ & $\Phi_{2} \begin{array}{c}\Delta \\
\text { differenza } \\
\text { per } 0.0001\end{array}$ & $\Phi_{3}$ & $\operatorname{tang} \alpha$ & $\mathbf{2} \Phi \begin{array}{c}\Delta \\
\text { differenza } \\
\text { per } 0.0001\end{array}$ & $\Phi_{3}$ \\
\hline 0.1810 & $\begin{array}{r}+1.8378 \\
-14.8\end{array}$ & +1.75 & 0.1945 & $\begin{array}{r}+1.6517 \\
-12.8\end{array}$ & +1.61 \\
\hline 0.1815 & $\begin{array}{ll}1.8304 & \\
& 14.8\end{array}$ & 1. 74 & 0.1950 & $\begin{array}{ll}1.6453 & \\
& 12.8\end{array}$ & 1. 60 \\
\hline 0.1820 & 1.8230 & 1. 74 & 0.1955 & 1. 6389 & 1. 60 \\
\hline 0.1825 & $\begin{array}{ll}1.8157 & \\
& 14.6\end{array}$ & 1. 73 & 0.1960 & 1. 6326 & 1. 59 \\
\hline 0.1830 & 1.8084 & 1. 73 & 0.1965 & 1. 6263 & 1. 59 \\
\hline 0.1835 & 1. 8012 & 1. 72 & 0.1970 & 1. 6200 & 1. 58 \\
\hline 0.1840 & 1. 7940 & 1. 72 & 0.1975 & 1. 6138 & 1. 58 \\
\hline 0.1845 & 1. 7868 & 1. 71 & 0.1980 & 1.6077 & 1. 57 \\
\hline 0.1850 & 1. 7797 & 1. 71 & 0.1985 & 1.6015 & 1.57 \\
\hline 0.1855 & 1. 7726 & 1. 70 & 0.1990 & 1. 5954 & 1. 56 \\
\hline 0.1860 & 1. 7656 & 1. 69 & 0.1995 & $\begin{array}{r}1.5893 \\
-12.2\end{array}$ & 1. 56 \\
\hline 0.1865 & 14.0 & 1. 69 & 0.2000 & +1.5832 differenza & +1.55 \\
\hline 0.1870 & 1. 7516 & 1. 68 & 0.200 & $+1.5832^{\text {per } 0.001}$ & +1.55 \\
\hline 0.1875 & 1. 7447 & 1. 68 & 0.201 & 1. 5712 & 1. 55 \\
\hline 0.1880 & 1. 7378 & 1. 67 & 0.202 & 1. 5593 & 1.54 \\
\hline 0.1885 & 1. 7310 & 1.67 & 0.203 & 1. 5475 & 1.53 \\
\hline 0.1890 & 1. 7242 & 1. 66 & 0.204 & 1. 5359 & 1. 52 \\
\hline 0.1895 & 1. 7174 & 1.66 & 0.205 & 1. 5244 & 1. 51 \\
\hline 0.1900 & 1. 7107 & 1.65 & 0.206 & 1. 5129 & 1. 50 \\
\hline 0.1905 & 1. 7040 & 1. 65 & 0.207 & 1.5016 & 1.49 \\
\hline 0.1910 & 1. 6973 & 1. 64 & 0.208 & 1. 4904 & 1. 48 \\
\hline 0.1915 & 1. 6907 & 1.64 & 0.209 & 1. 4793 & 1. 48 \\
\hline 0.1920 & 1. 6841 & 1. 63 & 0.210 & 1. 4683 & 1. 47 \\
\hline 0.1925 & 1. 6775 & 1. 63 & 0.211 & 1. 4574 & 1. 46 \\
\hline 0.1930 & 1.6710 & 1. 62 & 0.212 & 1. 4467 & 1.45 \\
\hline 0.1935 & 12. 8 & 1. 62 & 0.213 & 1. 4361 & 1. 44 \\
\hline 0.1940 & $\begin{array}{r}+1.6581 \\
-12.8\end{array}$ & $\div 1.61$ & 0.214 & $\begin{array}{r}+1.4255 \\
\end{array}$ & +1.43 \\
\hline
\end{tabular}


Continua Tab. III.

\begin{tabular}{|c|c|c|c|c|c|}
\hline $\operatorname{tang} \alpha$ & $\Phi_{2} \begin{array}{c}\text { differenza } \\
\text { per } 0.001\end{array}$ & $\Phi_{3}$ & $\operatorname{tang} \alpha$ & $\begin{array}{cc} & \Delta \\
\Phi_{2} & \text { differenza } \\
& \text { per } 0.001\end{array}$ & $\Phi_{3}$ \\
\hline 0.215 & $+1.4150-103$ & +1.43 & 0.242 & $+1.1667-81$ & +1.23 \\
\hline 0.216 & 1. 4047 & 1. 42 & 0.243 & 1. 1586 & 1. 23 \\
\hline 0.217 & $1.3945 \quad 102$ & 1. 41 & 0.244 & 1. $1506^{80}$ & 1. 22 \\
\hline 0.218 & 1. $3843^{102}$ & 1. 40 & 0.245 & 1. $1427^{79}$ & 1. 21 \\
\hline 0.219 & $\begin{array}{ll}1.3742 \quad 101 \\
\end{array}$ & 1. 39 & 0.246 & 1. $1348 \quad 79$ & 1. 21 \\
\hline 0.220 & 1. 3642 & 1. 39 & 0.247 & 1. 1270 & 1. 20 \\
\hline 0.221 & 1. 3543 & 1. 38 & 0.248 & 1. $1193^{77}$ & 1.19 \\
\hline 0.222 & 1. 3446 & 1. 37 & 0.249 & 1. 1116 & 1.19 \\
\hline 0.223 & 1. 3349 & 1. 36 & 0.250 & 1. $1040^{76}$ & 1. 18 \\
\hline 0.224 & 1. 3253 & 1.36 & 0.251 & $1.0965^{75}$ & 1. 18 \\
\hline 0.225 & 1. 3158 & 1. 35 & 0.252 & $1.0890^{75}$ & 1. 17 \\
\hline 0.226 & 1. 3063 & 1. 34 & 0.253 & 1.0816 & 1. 17 \\
\hline 0.227 & 1. 2970 & 1.33 & 0.254 & 1. 0742 & 1. 16 \\
\hline 0.228 & 1. $2878 \quad 92$ & 1.33 & 0.255 & 1.0669 & 1. 15 \\
\hline 0.229 & 1. $2786 \quad 92$ & 1.32 & 0.256 & 1.0596 & 1.15 \\
\hline 0.230 & 1. 2695 & 1. 31 & 0.257 & 1.0524 & 1.14 \\
\hline 0.231 & 1. 2605 & 1.30 & 0. 258 & $1.0453^{71}$ & 1. 14 \\
\hline 0.232 & 1. $2516^{89}$ & 1.30 & 0.259 & 1.0382 & 1.13 \\
\hline 0.233 & 1. $2428 \quad 88$ & 1.29 & 0.260 & 1.0312 & 1.13 \\
\hline 0.234 & 1. $2340^{88}$ & 1. 28 & 0.261 & 1.0242 & 1. 12 \\
\hline 0.235 & 1. 2253 & 1. 28 & 0.262 & 1.0173 & 1.12 \\
\hline 0.236 & 1. $2168^{85}$ & 1.27 & 0.263 & 1. 0105 & 1,11 \\
\hline 0.237 & $1.2083^{85}$ & 1,26 & 0.264 & $1.0037^{68}$ & 1,10 \\
\hline 0.238 & $1.1998^{85}$ & 1.26 & 0.265 & $0.9970^{67}$ & 1,10 \\
\hline 0.239 & 84 & 1.25 & 0.266 & 67 & 09 \\
\hline 0.259 & 1. 1914 & 1.20 & 0.266 & $\begin{array}{ll}0.9903 & \\
& \end{array}$ & \\
\hline 0.240 & 1. 1831 & 1. 24 & 0.267 & 0.9837 & 1.09 \\
\hline 0.241 & 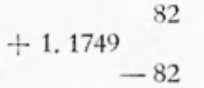 & +1.24 & 0.268 & $\begin{array}{r}66 \\
+0.9771-65\end{array}$ & +1.08 \\
\hline
\end{tabular}


Contimua Tab. III.

\begin{tabular}{|c|c|c|c|c|c|}
\hline $\operatorname{tang} \alpha$ & $\begin{array}{cc} & \Delta \\
(1) & \text { differenza } \\
\text { per } 0.001\end{array}$ & $(1) 3$ & $\operatorname{tang} \alpha$ & $\begin{array}{c} \\
\Phi_{2} \quad \begin{array}{c}\text { differenza } \\
\text { per } 0.001\end{array}\end{array}$ & $\Phi_{3}$ \\
\hline 0.269 & $\begin{array}{r}+0.9706 \\
-65\end{array}$ & +1.08 & 0.296 & $\begin{array}{r}+0.8120 \\
-53\end{array}$ & +0.95 \\
\hline 0.270 & 0.9641 & 1. 07 & 0.297 & $\begin{array}{ll}0.8067 & \\
52\end{array}$ & 0.95 \\
\hline 0.271 & $0.9577 \quad 64$ & 1.07 & 0.298 & $0.8015 \quad 52$ & 0.94 \\
\hline 0.272 & $0.9513 \quad 63$ & 1.06 & 0. 299 & $\begin{array}{l}0.7963 \\
-51\end{array}$ & 0.94 \\
\hline 0.273 & (). $9450 \quad 63$ & 1. 06 & 0.300 & +0.7912 & +0.94 \\
\hline 0.274 & $\begin{array}{ll}0.9387 & 62\end{array}$ & 1.05 & & $\begin{array}{r}\text { difierenza } \\
\text { per } 0.001\end{array}$ & \\
\hline 0.275 & $\begin{array}{ll}0.9325 \quad 62 & \end{array}$ & 1.05 & 0.300 & $\begin{array}{r}+0.7912 \\
-50.5\end{array}$ & +0.94 \\
\hline 0.276 & $\begin{array}{ll}0.9263 \quad 61 & \end{array}$ & 1.04 & 0.302 & $\begin{array}{ll}0.7811 \quad 50.5\end{array}$ & 0.93 \\
\hline 0.277 & 0.9202 & 1. 04 & 0.304 & $\begin{array}{ll}0.7710 & \\
& 49.5\end{array}$ & 0.92 \\
\hline 0.278 & $\begin{array}{ll}0.9141 & \\
& 61\end{array}$ & 1.03 & 0.306 & $\begin{array}{ll}0.7611 & \\
& 49.5\end{array}$ & 0.91 \\
\hline 0.279 & 0.9080 & 1.03 & 0.308 & $0.7512 \quad 48.5$ & 0.91 \\
\hline 0.280 & $0.9020 \quad 60$ & 1.02 & 0.310 & $\begin{array}{l}0.7415 \\
\end{array} 47.0$ & 0.90 \\
\hline 0.281 & $\begin{array}{ll}0.8960 & \\
& 59\end{array}$ & 1.02 & 0.312 & 0.7321 & 0.89 \\
\hline 0.282 & 0.8901 & 1. $(12$ & 0.314 & 0.7227 & 0.88 \\
\hline 0.283 & 0.8843 & 1.01 & 0.316 & 0.7134 & 0.88 \\
\hline 0.284 & 0.8785 & 1.01 & 0.318 & 45. 0 & 0.87 \\
\hline 0.285 & 0.8727 & 1.00 & 0.320 & 44.5 & 0.86 \\
\hline 0.286 & 0.8670 & 1.00 & 0. 322 & 44.0 & 0.85 \\
\hline 0.287 & $\begin{array}{ll}0.8613 \quad 56 & \end{array}$ & 0.99 & 0.324 & 43,0 & 0.84 \\
\hline 0.288 & 0.8557 & 0.99 & 0.326 & 0.6690 & 0.84 \\
\hline 0.289 & 0.8501 & 0.98 & 0.328 & 0.6605 & 0.83 \\
\hline 0.290 & 0.8445 & 0.98 & 0.330 & 41.5 & 0.82 \\
\hline 0.291 & 0.8390 & 0.97 & 0.332 & 0.6437 & 0.82 \\
\hline 0.292 & 0.8335 & 0.97 & 0.334 & 0.6355 & 0.81 \\
\hline 0.293 & 0. 8281 & (1. 97 & 0.336 & 0.6274 & 0.81 \\
\hline 0.294 & 0.8227 & 0.96 & 0.338 & 0.6195 & 0.80 \\
\hline 0.295 & $\begin{array}{r}+0.8173 \\
-53\end{array}$ & $\div 0.96$ & 0.340 & $\begin{array}{r}+0.6116 \\
-39.0\end{array}$ & $\div 0.79$ \\
\hline
\end{tabular}


Consinua Tab. III.

\begin{tabular}{|c|c|c|c|c|c|}
\hline $\operatorname{tang} \alpha$ & $\Phi_{2} \underset{\substack{\text { differenza } \\
\text { per } 0.001}}{\Delta}$ & $\Phi_{3}$ & $\operatorname{tang} \alpha$ & $\underset{\Phi_{2}}{\substack{\text { differenz: } \\
\text { per } 0.001}}$ & $\Phi_{3}$ \\
\hline 0.342 & $+0.6038-38.5$ & +0.78 & 0.396 & $+0.4265-27.5$ & +0.64 \\
\hline 0.344 & $\begin{array}{ll}0.5961 & \\
& 38.0\end{array}$ & 0.78 & 0.398 & $\begin{array}{ll}0.4210 & \\
& 27.5\end{array}$ & 0.63 \\
\hline 0.346 & $\begin{array}{ll}0.5885 & \\
& 37.5\end{array}$ & 0.77 & 0.400 & $\begin{array}{l}0.4155 \quad 27.0 \\
\end{array}$ & 0.63 \\
\hline 0.348 & $\begin{array}{l}0.5810 \quad 37.0 \\
\end{array}$ & 0.77 & 0.402 & $\begin{array}{ll}0.4101 & \\
& 26.5\end{array}$ & 0.63 \\
\hline 0.350 & $\begin{array}{ll}0.5736 & \\
& 36.5\end{array}$ & 0.76 & 0.404 & $\begin{array}{l}0.4048 \quad 26,5 \\
2\end{array}$ & 0.62 \\
\hline 0.352 & $\begin{array}{ll}0.5663 & \\
& 36.0\end{array}$ & 0.75 & 0.406 & $\begin{array}{ll}0.3995 \quad 26.0 & \end{array}$ & 0.62 \\
\hline 0.354 & $\begin{array}{ll}0.5591 & \\
& 35.5\end{array}$ & 0.75 & 0.408 & $\begin{array}{ll}0.3943 & \\
& 26.0\end{array}$ & 0.61 \\
\hline 0.356 & 0.5520 & 0.74 & 0.410 & $\begin{array}{ll}0.3891 & 25.5\end{array}$ & 0.61 \\
\hline 0.358 & $\begin{array}{ll}0.5450 & \\
& 34.5\end{array}$ & 0.74 & 0.412 & $\begin{array}{ll}0.3840 & \\
& 25.5\end{array}$ & 0.60 \\
\hline 0.360 & $\begin{array}{ll}0.5381 \quad 34.5\end{array}$ & 0.73 & 0.414 & 0.3789 & 0.60 \\
\hline 0.362 & 0.5312 & 0.72 & 0.416 & 0.3739 & 0.59 \\
\hline 0.364 & $0.5244 \quad 33.5$ & 0.72 & 0.418 & 0.3690 & 0.59 \\
\hline 0.366 & 0.5177 & 0.71 & 0.420 & 0.3641 & 0.59 \\
\hline 0.368 & $\begin{array}{ll}0.5111 \quad 32.5\end{array}$ & 0.71 & 0.422 & $\begin{array}{ll}0.3593 & \\
& 24.0\end{array}$ & 0.58 \\
\hline 0.370 & $\begin{array}{ll}0.5046 & \\
& 32.0\end{array}$ & 0.70 & $0 .+24$ & 0.3545 & 0.58 \\
\hline 0.372 & 0.4982 & 0.70 & 0.426 & 0.3498 & 0.57 \\
\hline 0.374 & 0.4918 & 0.69 & 0.428 & 0.3451 & 0.57 \\
\hline 0.376 & 0.4854 & 0.69 & 0.430 & 0.3404 & 0.57 \\
\hline 0.378 & 0.4792 & 0.68 & 0.432 & 0.3358 & 0.56 \\
\hline 0.380 & 30.5 & 0.68 & 0.434 & 0.3313 & 0.56 \\
\hline 0.382 & $\begin{array}{ll}0.4670 & \\
& 30.0\end{array}$ & 0.67 & 0.436 & 0.3268 & $0 . \overline{5}$ \\
\hline 0.384 & $\begin{array}{ll}0.4610 & \\
& 29.5\end{array}$ & 0.67 & 0.438 & 22.0 & 0.55 \\
\hline 0.386 & $\begin{array}{ll}0.4551 & \\
& 29.0\end{array}$ & 0.66 & 0.440 & 21.5 & $0.5 \overline{5}$ \\
\hline 0.388 & 0.4493 & 0.66 & 0.442 & 0.3137 & 0.54 \\
\hline 0.390 & 0.4435 & 0.65 & 0.444 & 0.3094 & 0.54 \\
\hline 0.392 & $\begin{array}{l}0.4378 \quad 28.5 \\
\end{array}$ & 0.65 & 0.446 & 0.3052 & 0.53 \\
\hline 0.394 & $\begin{array}{r}+0.4321-28.0 \\
-20\end{array}$ & -0.64 & 0.448 & $\begin{array}{r}+0.3010 \\
-21.0\end{array}$ & +0.53 \\
\hline
\end{tabular}


Continua Tab. III.

\begin{tabular}{|c|c|c|c|c|c|}
\hline $\operatorname{tang} \alpha$ & $\begin{array}{cc} & \Delta \\
\Phi_{0} \quad \begin{array}{c}\text { differenza } \\
\text { per } 0.001\end{array}\end{array}$ & $\Phi_{3}$ & $\operatorname{tang} \alpha$ & $\begin{array}{cc} & \Delta \\
\Phi_{2} & \begin{array}{c}\text { differenza } \\
\text { per } 0.001\end{array}\end{array}$ & $\Phi_{3}$ \\
\hline 0.450 & $\begin{array}{r}+0.2968 \\
-20.5\end{array}$ & +0.53 & 0.504 & $+0.1992-15.5$ & $\div 0.44$ \\
\hline 0.452 & 0.2927 & 0.53 & 0.506 & 0.1961 & 0.44 \\
\hline 0.454 & $\begin{array}{l}0.2886 \quad 20.0\end{array}$ & 0.52 & 0.508 & (). 1930 & 0.44 \\
\hline 0.456 & 0.2846 & 0.52 & 0.510 & 0.1899 & 0.43 \\
\hline 0.458 & $\begin{array}{ll}0.2806 & \\
& 20.0\end{array}$ & 0.51 & 0.512 & 0. 1869 & 0.43 \\
\hline 0.460 & $\begin{array}{l}0.2766 \quad 19.5 \\
\end{array}$ & 0.51 & 0.514 & 0. 1839 & 0.43 \\
\hline 0.462 & 0.2727 & 0.51 & 0.516 & 0. 1809 & 0.43 \\
\hline 0.464 & 0.2688 & 0.50 & 0.518 & 14. 5 & 0.42 \\
\hline 0.466 & 0.2650 & 0.50 & 0.520 & $\begin{array}{ll}0.1751 & \\
& 14.5\end{array}$ & 0.42 \\
\hline 0.468 & $\begin{array}{l}0.2612 \quad 18.5 \\
\end{array}$ & 0.50 & 0.522 & 0.1722 & 0.42 \\
\hline 0.470 & 0.2575 & 0.49 & 0.524 & 0.1693 & 0.42 \\
\hline 0.472 & 0.2538 & 0.49 & 0.526 & 0.1665 & 0.41 \\
\hline 0.474 & 0.2501 & 0.49 & 0.528 & 0.1637 & 0.41 \\
\hline 0.476 & 0.2465 & 0.48 & $0.531)$ & 0.1609 & 0.41 \\
\hline 0.478 & 0.2429 & 0.48 & 0.532 & 0.1581 & 0.41 \\
\hline 0.480 & 0.2393 & 0. 48 & 0.534 & 0. 1554 & 0.40 \\
\hline 0.482 & 17.5 & 0.47 & 0.536 & 0. 1527 & 0.40 \\
\hline 0.484 & 17.5 & 0.47 & 0.538 . & 0. 1500 & 0.40 \\
\hline 0.486 & 0. 2288 & ก. 47 & 0.540 & 0. 1474 & 0.40 \\
\hline 0.488 & 0.2254 & 0.46 & 0.542 & 0.1448 & 0.39 \\
\hline $0 .+90$ & 0. 2220 & 1). 46 & 0.544 & 13. 0 & 0.39 \\
\hline 0.492 & 0.2187 & 0.46 & 0.546 & 0.1396 & 0. 39 \\
\hline 0.494 & 0.2154 & 0.46 & 0.548 & 1). 1370 & 0.38 \\
\hline 0.496 & 0.2121 & 0.45 & 0.550 & 0.1345 & 0.38 \\
\hline 0.498 & ก. 2088 & 1). 45 & 0.552 & 12.5 & 0.38 \\
\hline 0.500 & 0.2056 & $0 .+5$ & 0.554 & 0.1295 & 0.38 \\
\hline 0.502 & $\begin{array}{r}+0.2024 \\
-16.0\end{array}$ & $\div 0.45$ & 0.556 & $\begin{array}{r}+0.1270 \\
-12.0\end{array}$ & +0.37 \\
\hline
\end{tabular}


Contimua Tab. III.

\begin{tabular}{|c|c|c|c|c|c|}
\hline $\operatorname{tang} \alpha$ & $\begin{array}{cc} & \Delta \\
\Phi_{2} & \text { differenza } \\
\text { per } 0.001\end{array}$ & $\Phi_{3}$ & $\operatorname{tang} \alpha$ & $\begin{array}{cc} & \Delta \\
\Phi_{2} & \text { differenza } \\
& \text { per } 0.001\end{array}$ & $\left(\mathrm{D}_{3}\right.$ \\
\hline 0.558 & $\begin{array}{r}+0.1246 \\
-12.0\end{array}$ & +0.37 & 0.625 & $\begin{array}{r}+0.0546 \\
-9.0\end{array}$ & +0.31 \\
\hline 0.560 & 0.1222 & 0.37 & 0.630 & 0.0501 & 0.30 \\
\hline 0.562 & 0.1198 & 0.37 & 0.635 & 0.0457 & 0.30 \\
\hline 0.564 & 0.1174 & 0.37 & 0.640 & 0.0415 & 0.29 \\
\hline 0.566 & 0.1150 & 0.36 & 0.645 & 0.0373 & 0.29 \\
\hline 0.568 & 0.1127 & 0.36 & 0.650 & 0.0332 & 0.29 \\
\hline 0.570 & 0.1104 & 0.36 & 0.655 & 0.0292 & 0.28 \\
\hline 0.572 & 0.1081 & 0.36 & 0.660 & 0.0253 & 0.28 \\
\hline 0.574 & 0.1058 & 0.36 & 0.665 & 0.0215 & 0.27 \\
\hline 0.576 & 0.1036 & 0.35 & 0.670 & 0.0178 & 0.27 \\
\hline 0.578 & 0.1014 & 0.35 & 0.675 & 0.0142 & 0.27 \\
\hline 0.580 & 0.0992 & 0.35 & 0.680 & 0.0106 & 0. 26 \\
\hline 0.582 & 0.0970 & 0.35 & 0.685 & 0.0071 & 0. 26 \\
\hline 0.584 & 0.0948 & 0.35 & 0.690 & 0.0037 & 0.26 \\
\hline 0.586 & 0.0926 & 0.34 & 0.695 & +0.01003 & 0.25 \\
\hline 0.588 & 0.0905 & 0.34 & 0.700 & -0.0030 & 0.25 \\
\hline 0.590 & $0.0884 \quad 10.5$ & 0.34 & 0.705 & 0.0062 & 0.25 \\
\hline 0.592 & 0.0863 & 0.34 & 0.710 & 0.0094 & 0. 24 \\
\hline 0.594 & 0.0842 & 0.34 & 0.715 & 0. 0125 & 0. 24 \\
\hline 0.596 & 0.0822 & 0.33 & 0.720 & 0.0155 & 0. 23 \\
\hline 0.598 & 0.0802 & 0.33 & 0.725 & 0.0184 & 0.23 \\
\hline 0.600 & +0.0782 & +0.33 & 0.730 & 0.0213 & 0.23 \\
\hline 0.600 & $+0.0782^{\text {per } 0.001}$ & +0.33 & 0.735 & 0.0241 & 0.23 \\
\hline 0.605 & 0.0732 & 0.33 & 0.740 & 0.0269 & 0.22 \\
\hline 0.610 & 0.0684 & 0.32 & 0.745 & 0.0296 & 0.22 \\
\hline 0.615 & 0.0637 & 0.32 & 0.750 & 0. 0323 & 0.22 \\
\hline 0.620 & $\begin{array}{r}+0.0591-2 \\
-9.0\end{array}$ & +0.31 & 0.755 & $\begin{array}{rr}-0.0349 & \\
& -5.2\end{array}$ & +0.21 \\
\hline
\end{tabular}


Contimu Tab. 111 .

\begin{tabular}{|c|c|c|c|c|c|}
\hline $\operatorname{tang} x$ & $\begin{array}{c}\Delta \\
\Phi_{2} \\
\substack{\text { differenza } \\
\text { per } 0.001}\end{array}$ & $\Phi_{3}$ & $\operatorname{tang} \alpha$ & $\begin{array}{cc} & \Delta \\
\Phi_{2} & \text { differenza } \\
\text { per } 0.001\end{array}$ & $\Phi_{3}$ \\
\hline 0.760 & -0.0375 - 5.0 & +0.21 & 0.895 & $\begin{array}{r}-0.0894 \\
-2.8\end{array}$ & $\div 0.15$ \\
\hline 0.765 & $0.0400+4.8$ & (0. 21 & 0.900 & $\begin{array}{ll}0.0908 & \\
& 2.8\end{array}$ & 0.14 \\
\hline 0.770 & (). 0424 4. 8 & 0. 21 & 0.905 & $\begin{array}{ll}0.0922 & \\
& 2.8\end{array}$ & 0. 14 \\
\hline 0. 775 & 0.0448 & (). 20 & 0.910 & $\begin{array}{l}0.0936 \quad 2.6\end{array}$ & 0.14 \\
\hline 0.780 & 0. 0471 & 0.20 & 0.915 & 0.0949 & 0.14 \\
\hline $\begin{array}{l}0.785 \\
0.790\end{array}$ & $\begin{array}{l}0.0494 \\
0.0517\end{array}$ & 0.20 & 0.920 & $\begin{array}{l}0.0962 \\
0.0975\end{array}$ & 0.14 \\
\hline 0.795 & 0.0539 & (0.19 & 0.930 & 2. 4 & 0.13 \\
\hline 0.800 & (0. 0560 & (). 19 & 0.935 & 0.0999 & 0.13 \\
\hline 0.805 & 0. 0581 & (0. 19 & 0.940 & 0.1011 & 0.13 \\
\hline 0.810 & 0. 0602 & 0. 18 & 0.945 & 0. 1023 & 0.13 \\
\hline 0.815 & 0.0622 & 0.18 & 0.950 & 0.1034 & 0.13 \\
\hline 0.820 & 0.0642 & 0. 18 & 0.955 & 0. 1045 & 0.12 \\
\hline 0. 825 & 0. 0661 & (). 18 & 0.960 & 0.1056 & 0.12 \\
\hline 0.830 & 0.0680 & 0. 18 & 0.965 & 0. 1067 & 0.12 \\
\hline 0. 835 & 0.0699 & 0.17 & 0.970 & 0. 1078 & 0.12 \\
\hline 0.840 & 0.0717 & 0.17 & 0.975 & (). 1088 & 0.12 \\
\hline 0.845 & 0.0735 & (). 17 & 0.980 & 0.1098 & 0.12 \\
\hline 0.850 & 0.0752 & 0. 17 & 0.985 & 0.1108 & 0.12 \\
\hline (1. 855 & $0 .(1769$ & 0. 16 & 0.990 & 0.1118 & 0.11 \\
\hline 0.860 & 0.0786 & 0. 16 & 0.995 & $0.1127-1.8$ & 0.11 \\
\hline 0.865 & 0.0802 & (J. 16 & 1.000 & -0.1136 & +0.11 \\
\hline 0.870 & 0.0818 & (J. 16 & & $\begin{array}{r}\text { differenza } \\
\text { per } 0.01\end{array}$ & \\
\hline (). 875 & 0.0834 & (). 15 & 1. 00 & $-0.1136-18$ & +0.11 \\
\hline 0.880 & (). 0850 & 0.15 & 1. 01 & $\begin{array}{ll}0.1154 \quad 17 & \end{array}$ & 0. 11 \\
\hline 0.885 & (). 0865 & 0. 15 & 1.02 & 0.1171 & 0.11 \\
\hline 0. 890 & $\begin{array}{r}-0.0880-2.8 \\
-2.8\end{array}$ & $\div 0.15$ & 1.03 & $-0.1187-15$ & +0.10 \\
\hline
\end{tabular}


Continua Tab. III.

\begin{tabular}{|c|c|c|c|c|c|}
\hline $\operatorname{tang} \alpha$ & $\begin{array}{cc} & \Delta \\
\bar{\Phi}_{2} & \text { differenza } \\
\text { per } 0.01\end{array}$ & $\Phi_{3}$ & $\operatorname{tang} \alpha$ & $\Phi_{2} \underset{\substack{\text { differenza } \\
\text { per } 0.01}}{\Delta}$ & $\Phi_{3}$ \\
\hline 1.04 & -0.1202 & +0.10 & 1. 31 & $-0.1+23$ & +0.05 \\
\hline 1.05 & 0.1217 & 0.10 & 1. 32 & 0.1426 & 0.05 \\
\hline 1.06 & 0.1231 & 0.10 & 1. 33 & 0.1429 & 0.05 \\
\hline 1.07 & 0.1245 & 0.09 & 1. 34 & 0.1431 & 0.04 \\
\hline 1.08 & 0.1258 & 0.09 & 1.35 & 0.1433 & 0.04 \\
\hline 1.09 & 0.1270 & 0.09 & 1.36 & 0.1435 & 0.04 \\
\hline 1. 10 & 0.1282 & 0.09 & 1. 37 & 0.1437 & 0.04 \\
\hline 1. 11 & 0.1293 & 0.08 & 1. 38 & 0.1439 & 0.04 \\
\hline 1. 12 & 0.1304 & 0.08 & 1. 39 & $0.1440^{1}$ & 0.04 \\
\hline 1. 13 & $0.1314^{10}$ & 0.08 & 1. 40 & $0.14+1 \quad 1$ & 0.04 \\
\hline 1.14 & 0.1323 & 0.08 & 1. 41 & $0.1442^{1}$ & 0.04 \\
\hline 1. 15 & 0.1332 & 0.08 & 1. 42 & 0.1443 & 0.03 \\
\hline 1. 16 & 0.1341 & 0.07 & 1. 43 & & 0.03 \\
\hline 1. 17 & 0.1349 & 0.07 & 1. 44 & $0.1444^{-1}$ & 0.03 \\
\hline 1. 18 & 0. 1357 & 0.07 & 1. 45 & & 0.03 \\
\hline 1. 19 & $0.1364^{7}$ & 0.07 & 1. 46 & & 0.03 \\
\hline 1.20 & $0.1371^{7}$ & 0.07 & 1. 47 & $0.1444^{0}$ & 0.03 \\
\hline 1. 21 & $0.1377^{6}$ & 0.06 & 1. 48 & & 0.03 \\
\hline 1. 22 & $0.1383^{6}$ & 0.06 & 1.49 & 0.1444 & 0.03 \\
\hline 1. 23 & 0.1389 & 0.06 & 1. 50 & $0.1+43^{+1}$ & 0.03 \\
\hline 1.24 & 0.1395 & 0.06 & 1. 51 & $0.1+42$ & 0.03 \\
\hline 125 & 0.1400 & 0.06 & 152 & 0.1441 & 0.02 \\
\hline 1.20 & $0.1+00$ & 0.00 & 1.22 & $0.1+41$ & \\
\hline 1. 26 & 0.1405 & 0.06 & 1. 53 & 0.1440 & 0.02 \\
\hline 1. 27 & $0.1409^{4}$ & 0.05 & 1.54 & 0.1439 & 0.02 \\
\hline 1. 28 & $0.1413^{4}$ & 0.05 & 1.55 & 0.1438 & 0.02 \\
\hline & 4 & & & $\mathbf{i}$ & \\
\hline 1. 29 & 0.1417 & 0.05 & 1. 56 & $0.1+37$ & 0.02 \\
\hline 1. 30 & $\begin{array}{r}3 \\
-0.1420^{3} \\
-3\end{array}$ & +0.05 & 1.57 & $-0.1436 \begin{array}{r}1 \\
+2\end{array}$ & $-1-0.02$ \\
\hline
\end{tabular}


Continua Tab. III.

\begin{tabular}{|c|c|c|c|c|c|}
\hline $\operatorname{tang} \alpha$ & $\underset{\substack{\text { differenza } \\
\text { per } 0.01}}{\Delta}$ & $D_{3}$ & tang $\alpha$ & $\begin{array}{r}\Delta \\
\Phi_{2} \text { differenza } \\
\text { per } 0.1\end{array}$ & $\Phi_{3}$ \\
\hline 1. 58 & -0.1434 & +0.02 & 2. 1 & -0.1273 & 0.00 \\
\hline 1.59 & $0.1432^{+2}$ & 0.02 & 2. 2 & $0.1235^{+3 x}$ & 1). 00 \\
\hline 1.60 & $-0.1430^{+2}$ & +0.02 & 2.3 & $0.1197^{38}$ & -0.01 \\
\hline & $\begin{array}{r}\text { differenza } \\
\text { per } 0.01\end{array}$ & & 2. 4 & $\begin{array}{ll}0.1159 & \\
& 37\end{array}$ & 0.01 \\
\hline 1.60 & $\begin{array}{r}-0.1430 \\
+2.0\end{array}$ & +0.02 & 2.5 & $\begin{array}{ll}0.1122 & \\
& \end{array}$ & 1). 01 \\
\hline 1. 62 & 0.1426 & 0.02 & 2.6 & 0. 1087 & 0.01 \\
\hline 1.64 & 0.1422 & 0.02 & 2.7 & 0.1052 & 0.01 \\
\hline 1.66 & 0.1418 & 0.01 & 2.8 & 0.1018 & 0.01 \\
\hline 1.68 & 0.1413 & 0.01 & 2.9 & 0.0985 & 0.01 \\
\hline 1. 70 & 0.1408 & 0.01 & $3.1)$ & 0.0953 & 0.01 \\
\hline 1.72 & 0.1403 & 0.01 & 3.1 & 0.0922 & 0.01 \\
\hline 1. 74 & 0.1397 & 0.01 & 3.2 & 0.0893 & 0.01 \\
\hline 1.76 & 0.1391 & 0.01 & 3.3 & 0.0865 & 0.01 \\
\hline 1.78 & $0.1385 \quad 3.0$ & 0.01 & 3.4 & 0. 0838 & 0.01 \\
\hline 1.80 & 0.1379 & 0.01 & 3.5 & 0.0812 & 0.01 \\
\hline 1.82 & 0.1373 & 0. 01 & 3.6 & 0.0787 & 0.01 \\
\hline 1.84 & 0.1367 & +0.01 & 3.7 & 0.0764 & 0.01 \\
\hline 1.86 & 0.1360 & 0.00 & 3.8 & 0.0741 & 0.01 \\
\hline 1.88 & 0.1353 & (1). 00 & 3.9 & 0.0719 & 0.01 \\
\hline 1.90 & 0.1346 & 0.00 & 4.0 & 0.0698 & ). 01 \\
\hline 1.92 & $0.1339^{3.5}$ & 0.00 & 4. 1 & 0.0678 & 0.01 \\
\hline 1. 94 & 0.1332 & 0.00 & 4. 2 & 0.0659 & 0.01 \\
\hline 1.96 & 0.1325 & 0.00 & 4.3 & 0.0641 & 0.01 \\
\hline 1.98 & 0.1318 & 0.00 & 4. 4 & 0.0623 & 0.01 \\
\hline 2. 00 & $-0.1310^{+4.0}$ & 0.00 & 4.5 & 0.0606 & 0.01 \\
\hline & $\begin{array}{r}\text { differenza } \\
\text { per } 0.1\end{array}$ & & 4.6 & $\begin{array}{ll}0.0590 & 16 \\
& 15\end{array}$ & 0.01 \\
\hline 2.0 & $\begin{array}{r}-0.1310 \\
+37\end{array}$ & 0.00 & 4.7 & $\begin{array}{r}-0.057+ \\
+15\end{array}$ & -0.01 \\
\hline
\end{tabular}


Continua Tab. III.

\begin{tabular}{|c|c|c|c|c|c|}
\hline $\operatorname{tang} \alpha$ & $\begin{array}{c}\Delta \\
\Phi_{2} \quad \begin{array}{c}\text { differenza } \\
\text { per } 0.1\end{array}\end{array}$ & $\Phi_{3}$ & $\operatorname{tang} \alpha$ & $\begin{array}{c}\Delta \\
\Phi_{2} \text { differenza } \\
\text { per } 0.1\end{array}$ & $\Phi_{3}$ \\
\hline 4. 8 & $\begin{array}{r}-0.0559+14 \\
+14\end{array}$ & -0.01 & 7.5 & $-0.0306+6$ & -0.01 \\
\hline 4. 9 & 0.0545 & 0.01 & 7.6 & 0.0300 & 0.01 \\
\hline 5.0 & 0.0531 & 0.01 & 7.7 & 0.0294 & 0.01 \\
\hline 5.1 & 0.0518 & 0.01 & 7. 8 & 0.0289 & 0.01 \\
\hline 5.2 & 0.0505 & 0.01 & 7.9 & 0.0284 & 0.01 \\
\hline 5.3 & 0.0492 & 0.01 & 8. 0 & 0.0279 & -0.01 \\
\hline 5.4 & 0.0480 & 0.01 & 8.1 & 0.0274 & 0.00 \\
\hline 5.5 & 0.0469 & 0.01 & 8.2 & 0.0269 & 0.00 \\
\hline 5.6 & 0.0458 & 0.01 & 8.3 & 0.0264 & 0.00 \\
\hline 5.7 & $0.0447 \quad 11$ & 0.01 & 8.4 & 0.0259 & 0.00 \\
\hline 5.8 & 0.0437 & 0.01 & 8.5 & 0.0255 & 0.00 \\
\hline 5.9 & 0.0427 & 0.01 & 8.6 & 0.0251 & 0.00 \\
\hline 6.0 & 0.0417 & 0.01 & 8.7 & 0.0246 & 0.00 \\
\hline 6.1 & $\begin{array}{ll}0.0408 & \end{array}$ & 0.01 & 8.8 & 0.0242 & 0.00 \\
\hline 6.2 & 0.0399 & 0.01 & 8.9 & 0.0238 & 0.00 \\
\hline 6.3 & 0.0390 & 0.01 & 9.0 & 0.0234 & 0.00 \\
\hline 6.4 & $\begin{array}{ll}0.0382 & \end{array}$ & 0.01 & 9.1 & 0.0231 & 0.00 \\
\hline 6.5 & $\begin{array}{ll}0.0374 & \end{array}$ & 0.01 & 9.2 & 0.0227 & 0.00 \\
\hline 6.6 & $\begin{array}{ll}0.0366 & \\
& 7\end{array}$ & 0.01 & 9.3 & 0.0223 & 0.00 \\
\hline 6.7 & $\begin{array}{ll}0.0359 & \\
& 8\end{array}$ & 0.01 & 9.4 & 0.0220 & 0.00 \\
\hline 6.8 & 0.0351 & 0.01 & 9.5 & $\begin{array}{ll}0.0216 \quad 3 & \end{array}$ & 0.00 \\
\hline 6.9 & 0.0344 & 0.01 & 9.6 & 0.0213 & 0.00 \\
\hline 7.0 & 0.0337 & 0.01 & 9.7 & $0.0210_{4}$ & 0.00 \\
\hline 7.1 & 0.0330 & 0.01 & 9.8 & 0.0206 & 0.00 \\
\hline 7.2 & 0.0324 & 0.01 & 9.9 & $0.0203+3$ & 0.00 \\
\hline 7.3 & 0.0318 & 0.01 & 10.0 & -0.0200 & 0.00 \\
\hline 7.4 & $\begin{array}{r}-0.0312+6 \\
+6\end{array}$ & -0.01 & & & \\
\hline
\end{tabular}


Continta Tab. III.

\begin{tabular}{|c|c|c|c|c|c|}
\hline $\operatorname{tang} \alpha$ & $\underset{\Phi_{2} \text { differenza }}{\Delta} \underset{\text { per } 0.1}{\Delta}$ & $\Phi_{3}$ & $\operatorname{tang} \alpha$ & $\underset{\Phi_{2} \text { differenza }}{\text { per } 0.1}$ & $\Phi_{3}$ \\
\hline 10.0 & $-0.0200+3.0$ & 0.00 & 13.8 & $-0.0122+1.5$ & 0.00 \\
\hline 10.2 & 0.0194 & 0.00 & 14. 0 & 0.0119 & 0.00 \\
\hline 10.4 & 0.0189 & 0.00 & 14.2 & 0.0117 & 0.00 \\
\hline 10.6 & 0.0183 & 0.00 & 14. 4 & 0.0114 & 0.00 \\
\hline 10.8 & 0.0178 & 0.00 & 14.6 & 0.0112 & 0.00 \\
\hline 11.0 & 0.0173 & 0.00 & 14.8 & 0.0109 & 0.00 \\
\hline 11. 2 & 0.0169 & 0.00 & 15.0 & $-0.0107^{+1.0}$ & 0.00 \\
\hline 11.4 & $\begin{array}{l}2.5 \\
2.0\end{array}$ & 0.00 & & $\begin{array}{r}\text { differenza } \\
\text { per } 0.1\end{array}$ & \\
\hline 11.6 & 0.0160 & 0.00 & 15.0 & $\begin{array}{l}-0.0107+1.2 \\
+1.2\end{array}$ & 0.00 \\
\hline 11.8 & 0.0156 & 0.00 & 15.5 & $\begin{array}{ll}0.0101 \quad 1.0 & \end{array}$ & 0.00 \\
\hline 12. 0 & 0.0152 & 0.00 & 16.0 & 0.0096 & 0.00 \\
\hline 12. 2 & 0.0148 & 0.00 & 16.5 & 0.0092 & 0.00 \\
\hline 12. 4 & 0.0144 & 0.00 & 17.0 & 0.0087 & 0.00 \\
\hline 12.6 & 0.0141 & 0.00 & 17. $\overrightarrow{5}$ & 0.0083 & 0.00 \\
\hline 12.8 & 0.0137 & 0.00 & 18.0 & 0.0080 & 0.00 \\
\hline 13.0 & 0.0134 & 0.00 & 18.5 & 0.0076 & 0.00 \\
\hline 13. 2 & 0.0131 & 0.00 & 19.0 & $\begin{array}{ll}0.0073 & \\
& 0.6\end{array}$ & 0.00 \\
\hline 13.4 & 0.0128 & 0.00 & 19.5 & $\begin{array}{r}0.0070 \\
+0.6\end{array}$ & 0.00 \\
\hline 13.6 & $\begin{array}{r}-0.0125+1.5 \\
+1.5\end{array}$ & 0.00 & 20.0 & -0.0067 & 0.00 \\
\hline
\end{tabular}


Cosi per $d \leqslant 500 \mathrm{~km}$ e per $|H|<70 \mathrm{~km}$ con un errore assoluto dell'ordine di $0.4 \quad 10^{-5}$ gal la [12] si riduce all'espressione:

$$
F=-2 \pi k R ; \Phi_{1}(\alpha) y-\left[\Phi_{*}(\alpha) y^{2}+\Phi_{3}(\alpha) y^{3}\right]^{\prime}
$$

5. - Per $\operatorname{tang} \alpha<0.2$ si ha sviluppando in serie le [11] e tenendo l'approssimazione di $0.210^{-5}$ gal per valori $H$ non superiori in valor aritmetico a $70 \mathrm{~km}$,

$$
\begin{aligned}
& 2 \pi k R y \Phi_{1}(\alpha)=2 \pi k R y\left[1-\frac{1}{2} \operatorname{tang} \alpha+\frac{1}{8} \operatorname{tang}^{3} \alpha-\frac{1}{16} \operatorname{tang}^{5} \alpha+\ldots \ldots \ldots\right] \\
& 2 \pi k R y^{2} \Phi_{9}(\alpha)=2 \pi k R y^{2}\left[\frac{1}{2} \operatorname{cotg} \alpha-1+\frac{5}{12} \operatorname{tang} \alpha-\frac{1}{80} \tan ^{3} \alpha+\ldots \ldots \ldots\right] \\
& 2 \pi k R y^{3} \Phi_{3}(\alpha)=2 \pi k R y^{3}\left[\frac{3}{8} \operatorname{cotg} \alpha-\frac{1}{3}+\frac{1}{16} \operatorname{tang} \alpha+\ldots \ldots \ldots\right] \\
& 2 \pi k R y^{4} \Phi_{4}(\alpha)=2 \pi k R y^{4}\left[\frac{1}{16} \operatorname{cotg} \alpha-\frac{1}{160} \operatorname{tang} \alpha+\ldots \ldots \ldots\right] \\
& 2 \pi k R y^{5} \Phi_{5}(\alpha)=2 \pi k R y^{5}\left[-\frac{1}{128} \operatorname{cotg} \alpha+\ldots \ldots \ldots\right]
\end{aligned}
$$

e quindi per tang $\alpha \leqslant 0.1$ la [12] con l'approssimazione di $0.110^{-5}$ gal, si può scrivere, per tutti $i$ valori di $x$ compresi fra 0 e 1 e per |H $\mid \leqslant 70 \mathrm{~km}$.

$$
\begin{gathered}
F=-2 \pi k R\left[\left(1-\frac{1}{2} \operatorname{tang} \alpha+\frac{1}{8} \operatorname{tang}^{3} \alpha-\frac{1}{16} \operatorname{tang}^{5} \alpha\right) y-\right. \\
-\left(\frac{1}{2} \operatorname{cotg} \alpha-1+\frac{5}{12} \operatorname{tang} \alpha-\frac{1}{80} \operatorname{tang}^{3} \alpha\right) y^{\prime \prime}- \\
-\left(\frac{3}{8} \operatorname{cotg} \alpha-\frac{1}{3}+\frac{1}{16} \operatorname{tang} \alpha\right) y^{2} \\
\left.-\frac{1}{16} \operatorname{cotg} \alpha y^{4}+\ldots \ldots \ldots\right)
\end{gathered}
$$

Ponendo in questa $x=1$ si ha l'attrazione esercitata dall'intero guscio sferico su di un punto della sua superficie esterna o interna a seconda che $H$ è negativo o positivo:

$$
\begin{gathered}
F(-|y|)=4 \pi k R|y|\left(1-|y|+\frac{1}{3} \cdot y^{2}-\frac{1}{128}|y|^{3}\right) \\
F(|y|)=2 \pi k R \frac{1}{64} y^{4}
\end{gathered}
$$


che differiscono dai valori esatti che si ottengono dalle [12]:

$$
F(-|y|)=4 \pi k R|y|\left(1-|y|+\frac{y^{2}}{3}\right) \quad F(|y|)=0
$$

a meno della quantità $2 \pi k R \frac{y^{4}}{64}$ che per $|H| \leqslant 100 \mathrm{~km}$ non supera $0.0310^{-5} \mathrm{gal}$.

6. - Inoltre ordinando le precedenti secondo le potenze ascendenti di $y$ si ottiene

per valori positivi di $y$

$$
\begin{gathered}
F(|y|)=-2 \pi k R\left[(1-x)|y|-\left(\frac{1+3 x^{2}}{4 x}-1\right) y^{2}-\left(\frac{5+3 x^{2}}{24 x}-\frac{1}{3}\right)\left|y^{3}\right|+\right. \\
\left.+\frac{\left(1-x^{2}\right)^{2}}{64 x^{3}} y^{4}+\frac{\left(1+3 x^{2}\right)\left(1-x^{2}\right)}{640 x^{3}}\left|y^{5}\right|-\frac{\left(3+2 x^{2}+3 x^{4}\right)\left(1-x^{2}\right)}{1536 x^{5}} y^{6}+\ldots\right]
\end{gathered}
$$

[12"']

per valori negativi di $y$

$$
\begin{gathered}
F(-\mid y)=-2 \pi k R\left[-(1+x)|y|+\left(\frac{1+3 x^{2}}{4 x}+1\right) y^{2}-\left(\frac{5+3 x^{2}}{24 x}-\frac{1}{3}\right) \mid y^{3}-\right. \\
\left.-\frac{\left(1-x^{2}\right)^{2}}{64 x^{3}} y^{-4}+\frac{\left(1+3 x^{2}\right)\left(1-x^{2}\right)}{640 x^{3}} y^{5}+\frac{\left(3+2 x^{2}+3 x^{4}\right)\left(1-x^{2}\right)}{1536 x^{5}} y^{6}+\ldots\right]
\end{gathered}
$$

7. - In fine per $\operatorname{tang} \alpha \geqslant 5(\operatorname{cotg} \alpha \leqslant 0.2)$ si ha $\Phi_{1}(\alpha)=\operatorname{cotg} \alpha-\frac{1}{2} \operatorname{cotg}^{0} \alpha+\frac{1}{8} \operatorname{cotg}^{4} \alpha-\frac{1}{16} \operatorname{cotg}^{6} \alpha+\frac{5}{128} \operatorname{cotg}^{8} \alpha+\ldots \ldots \ldots$ $\Phi_{\mathrm{a}}(\alpha)=\operatorname{cotg}^{\mathrm{a}} \alpha \ln \left(\frac{1}{2} \operatorname{cotg} \alpha\right)+\operatorname{cotg}^{2} \alpha-\frac{5}{8} \operatorname{cotg}^{4} \alpha+\frac{11}{32} \operatorname{cotg}^{0} \alpha+\ldots \ldots$

$\Phi_{3}(\alpha)=-\frac{1}{2} \operatorname{cotg}^{2} \alpha+\frac{5}{3} \operatorname{cotg}^{3} \alpha-\frac{3}{2} \operatorname{cotg}^{4} \alpha+\ldots \ldots \ldots$

$\Phi_{4}(\alpha)=-\left[\frac{3}{4} \ln \left(\frac{1}{2} \ldots \ldots, \ldots\right)+1\right] \operatorname{cotg}^{4} \alpha+\ldots \ldots \ldots$

e quindi con la stessa approssimazione tenuta nel ricavare la [12'], rimanendo ferma la condizione $|H| \leqslant 70 \mathrm{~km}$, si ricava:

$$
\begin{aligned}
F= & -2 \pi k R y\left[\left(\operatorname{cotg} \alpha-\frac{1}{2} \operatorname{cotg}^{2} \alpha+\frac{1}{8} \operatorname{cotg}^{4} \alpha-\frac{1}{16} \operatorname{cotg}^{6} \alpha\right) y-\right. \\
& -\left(\operatorname{cotg}^{n} \alpha \ln \left[\frac{1}{2} \operatorname{cotg} \alpha\right]+\operatorname{cotg}^{2} \alpha-\frac{5}{8} \operatorname{cotg}^{4} \alpha\right) y^{2}+ \\
& \left.+\left(\frac{1}{2} \operatorname{cotg}^{2} \alpha-\frac{5}{3} \operatorname{cotg}^{3} \alpha\right) y^{3}+\ldots \ldots \ldots\right]
\end{aligned}
$$


8. - I numeri di Cassinis. Per definizione il numero $f$ di Cassinis relativo ad una zona linitata dai valori angolari $\phi_{1}<\phi_{2}$ e compresa tra la quota zero e la quota $H$ riferite alla sfera di raggio $R=6371.2 \mathrm{~km}$, viene espresso, in unita di $1.10^{-5}$ gal. dalla differenza

se la quota $H$ è positiva $f(y)=F\left(x_{1}, y\right)-F\left(x_{2}, y\right)$

$" \quad " \quad H$ is negativa $\quad f(y)=F\left(x_{2}, y\right)-F\left(x_{1}, y\right)$

sicché per ottenere in generale l'attrazione cambiata di segno esercitata dalla zona compresa tra le quote $H_{1}<H$, sulla massa unitaria posta nel punto $S$ di quola zero, e cioè la riduzione $\Delta f$ da applicare al valore della gravita osservata in $S$, occorre fare la differenza:

$$
f\left(y_{2}\right)-f\left(y_{1}\right)
$$

per poi moltiplicarla per la densità $\delta$ della materia costituente la zona:

$$
y_{1}<y_{2} \quad \Delta f=\delta\left[f\left(y_{2}\right)-f\left(y_{1}\right)\right]
$$

Come facilmente si deduce dalle [12], [12'], la funzione $F$ i positiva e crescente col crescere di $x$, per valori negativi della quota $H$, mentre per valori positivi di $I f$ essa è negativa ed assume il massimo valore negativo quando, con sufficiente approssimazione e per valori di $H$ che interessano le riduzioni, $x=\frac{1}{2} \sqrt{y}$.

Sicché i numeri di Cassinis relativi a valori negativi delle quote risultano sempre positivi; mentre per una quota positiva sono positivi per le zone comprese fra $x=0$ e $x=\frac{1}{2} \sqrt{y}$, negativi per le altre.

Così ad esempio a partire dalla zona 18 tutti i numeri $f$ corrispondenti a valori di $H F$ positivi ed inferiori a $5000 \mathrm{~m}$, sono negativi.

9. - Esempio: Calcolare il numero di Cassinis relativo alla zona $M$, che è compresa fra: $x_{1}=0.0022601688 x_{2}=0.0046144989$, di quota $H=-50 \mathrm{~km}$.

Assumendo, come nelle Tavole fondamentali per la riduzione dei vulori osservati della gravità, per il raggio della sfera di riferimento dolle quote il raggio medio $R=6371.2 \mathrm{~km}$ dell'ellissoide internazionale, e per la costante $k$ dell'attrazione universale il valore $6,6710^{-s}$ c.r.s. si ha applicando la [12]: 
$I=-50 \mathrm{~km} \quad y=\frac{H}{R}=-0.007 \quad 8478 \quad 152 \quad 2 \pi k R \quad y=-2.0954423$

$$
2 \pi k R y^{-2}=+0.0164446 \quad 2 \pi k R y^{* 3}=-0.000 \quad 3291
$$

per $x_{1}=0.0022601688$

$$
\operatorname{tang} \alpha=\frac{|y|}{2 x_{1}}=1.73611263 \quad \operatorname{tang} x=\frac{|y|}{2 x_{y}}=0.85031316
$$

Con questi valori dalle menzionate tabelle di Brandicourt e Roussilhe si ricava successivamente:

$$
\begin{aligned}
\alpha & =66^{\circ} .731199 & \varkappa & =44^{\circ} .862166 \\
\operatorname{tang} \frac{\alpha}{2} & =0.57802625 & \text { tang } \frac{\alpha}{2} & =0.36769008
\end{aligned}
$$

c quindi :

や $=1-\operatorname{tang} \frac{\alpha}{2}=0.42197375 \quad \Phi_{\mathrm{i}}=1-\operatorname{tang} \frac{\alpha}{2}=0.63230992$

mentre dalle allegate tabelle III si ottengono, con l'argomento tang $\%$, i valori :

$\Phi_{2}=-0.1398 \quad \Phi_{:}=+0.01 \quad \Phi_{: 2}=-0.0753 \quad \Phi_{: 2}=+0.17$

e quindi dalla [12] si ha in unita di gal:

$-2 \pi k R y \omega_{1}(x)=+0.88+222 \quad-2 \pi k R y$ ( $_{1}(x)=+1.324969$

$\left.+2 \pi k R y^{*}\right)_{0}(\alpha)=-0.002299+2-k R y^{\circ}()_{2}(\alpha)=-0.001238$

$+2 \pi k R y^{3}\left(\mathrm{~J}_{3}(x)=-0.000001 \quad+2 \pi k R y^{3} W_{3}(\alpha)=-0.000022\right.$

$F\left(x_{1}, y\right)=+0.881922 \quad F(x \cdot y)=+1.323 \quad 709$

Infine dalla seconda delle [13]

$$
I=F(x, y)-F\left(x_{1}, y\right)=+41178.7
$$

valore che differisce di 0.1 da quello che si ottiene applicando la formola rigrorosa di Cassinis.

Analogamente se $I=+10 \mathrm{~km}$ si ricava sempre con la [12]:

$$
\begin{aligned}
& I=+10 \mathrm{~km} \quad y=+0.0015695630 \quad 2-k R y=+0.4190885 \\
& 2 \pi k y^{\prime \prime}=+0.0006578 \quad 2 \pi k y^{* i}=+0.0000010 \\
& \operatorname{tang} x=0.3472 \text { 2252 } \quad \operatorname{tang} x=0.17006863 \\
& \alpha=21 .=275 \text { 725 } \quad \alpha=10=72+296 \\
& \operatorname{tang} \frac{\tilde{\alpha}}{\ddot{\alpha}}=0.16867198 \quad \text { tang } \frac{\alpha}{2}=0.08412818 \\
& \mathrm{D}_{1}=0.831328112 \quad \Phi_{1}=0.91557182
\end{aligned}
$$

$\Phi_{2}=+0.5839 \quad \coprod_{: 3}=+0.77 \quad \Phi_{2}=+2.0108 \quad \Phi_{3}=+1.88$ 
$-2 \pi k R y \mathrm{~W}_{1}(\alpha)=-0.348400$

$+2 \pi k R y^{2} \Phi,(\alpha)=+0.000384$.

$+2 \pi k R y^{3} \Phi_{:}(\alpha)=+0.000001$

$$
F\left(x_{1}, y\right)=-0.348015
$$

$$
\begin{aligned}
& -2 \pi k R \cdot \omega_{1}(\alpha)=-0.383706 \\
& +2 \pi k R \gamma^{2}()_{,}(\alpha)=+0.001323 \\
& +2 \pi R R y^{3} \mathrm{D}_{3}(\alpha)=+0.000002 \\
& F(x: y)=-0.382381
\end{aligned}
$$

e quindi per la prima delle [13]

$$
f=F\left(x_{1} y\right)-F\left(x_{2}, y\right)=+3436.6
$$

che coincide con il valore che si ricava impiegando la formola chiusa.

10. - Ponendo per semplicità di scrittura:

$$
\begin{aligned}
& n=x_{:}-x_{1} \\
& a=\frac{\left(1-x_{2}^{2}\right)^{2}}{64 x_{1}^{3}}-\frac{\left(1-x_{2}^{2}\right)^{2}}{64 x_{2}^{3}} \\
& b=\frac{1+3 x_{1}^{2}}{4 x_{1}}-\frac{1+3 x_{2}^{2}}{4 x^{2}} \quad e=\frac{\left(1+3 x_{1}{ }^{2}\right)\left(1-x_{1}{ }^{2}\right)}{640 x^{3}}-\frac{\left(1+3 x_{2}^{2}\right)\left(1-x_{2}^{2}\right)}{640 x_{2}{ }^{3}} \\
& c=\frac{5+3 x_{1}^{2}}{21 x_{1}}-\frac{5+3 x_{2}{ }^{2}}{24 x_{z}} \quad f=\frac{\left(3-2 x_{1}{ }^{2}+3 x_{1}{ }^{4}\right)\left(1-x_{1}{ }^{2}\right)}{1536 x_{1}{ }^{5}}-\frac{\left(3+2 x_{2}+3 x_{2}{ }^{4}\right)\left(1-x_{2}\right.}{1536 x_{2}{ }^{5}}
\end{aligned}
$$

dalle $\left(12^{\prime \prime \prime}\right)$ si ottiene infine la seguente espressione per il numero di

\begin{tabular}{|c|c|c|c|c|}
\hline Zoma & $\mathrm{a}$ & $b$ & $c$ & d \\
\hline 10 & +0.025006 & +0.956682 & $-0.8097+$ & -29.343 \\
\hline 9 & 0.029639 & +0.620995 & 0.53232 & 10.655 \\
\hline 8 & 0.056349 & +0.594858 & (1. 52389 & 5. 580 \\
\hline 7 & 0.051240 & +0.270802 & 0.25129 & 1.390 \\
\hline 6 & 0.077 .983 & +0.215160 & 0.21729 & (1. 70$) 5$ \\
\hline 5 & 0.122296 & +0.138022 & 0.17617 & 0.200 \\
\hline 4 & 0.158278 & +0.037067 & 0.11003 & 0.096 \\
\hline 3 & 0. 208270 & -0.045427 & 0.06628 & (1). 0213 \\
\hline 2 & $0.170+18$ & -0.072630 & 0.02468 & +0.0104 \\
\hline 1 & -0.031998 & -0.015735 & +0.00289 & $0 .(1) 0$ \\
\hline
\end{tabular}
Cassinis relativo ad una zona generica

$f(y)=-2 \pi k R y\left[a-b y-c y^{2}+d y^{3}+e y^{4}-f y^{5}+\ldots \ldots \ldots\right]$

attribuendo, come nelle (12), alla $y$ il segno che le compete: positivo se $H>o$. negativo nel caso contrario.

I valori dei coefficienti per le ultime zone della suddivisione di Hayford, per le quali lo sviluppo si riduce ai primi termini scritti, sono contenuti nella seguente tabella.

TABELLA IV

4. - Variazione dei nurieri di Cassinis per una variazione del raggio DELLA SFERA DI RIFERIMENTO.

1. - Fin qui si ¿̀ supposto il punto potenziato $S$ sulla superficie di riferimento delle quote, schematizzata dalla sfera di raggio $R$. Nel caso generale di una stazione gravimetrica di altitudine $\boldsymbol{H}_{\mathrm{s}}$, l'attra- 
zione della calotta di spessore $I I$, compresa ciò tra le sfere di raggio $R$ e $R-I I$. si otterà evidentemente sommando o sottraendo, secondo il segno di $H_{\mathrm{s}}$, le attrazioni dovute alle due calotte di spessore rispetlivamente uguale a: $h_{. .}=H-H_{s}: h_{1}=-H_{\mathrm{s}}$. La superficie in comune delle due calotte contiene il punto potenziato e quindi le loro attrazioni si calcoleranno con le formole [12] nelle quali però al ragrgio $R$ andrà sostituito il raggeio $r=R+H_{\mathrm{s}}$.

Se peraltro supponiamo di aver calcolato, prendendo come argomento la quota, i valori di $F$ relativi alla sfera di raggro $R$, sarà necessario. per tener conto dell'altezza della stazione, determinare la correzione $"$ da apportare a quei valori per passare al caso della sfera di raggio $r=R+H_{\mathrm{s}}$.

Indichiamo perciò con

$$
Y-\frac{H}{r}=y\left(1+y_{\mathrm{s}}\right)^{-1}=y-y y_{\mathrm{s}}
$$

- avendo posto $y_{\mathrm{s}}=\frac{H_{*}}{R}$ e trascurato rispetto all'unità le seconde potenze di $y_{\mathrm{s}}$-.. e sviluppiamo, con la formola di Taylor, la funzione $F$. espressa dalla [12], relativa alla sfera di raggio $r$, si ottiene:

$F(x, Y)=F\left(x, y-y y_{\mathrm{s}}\right)=F(x, y)-2 \pi k R y y_{\mathrm{s}} \cos \alpha \tan g \frac{\alpha}{2}+\ldots \ldots \ldots$ a meno di termini il cui contributo è dell'ordine di $2-k R y^{2} y_{\mathrm{s}}$.

Quindi la correzione da apportare al valore $F(x, y)$ per una variazione $I I$, del raggio $R$ della sfera risulta:

$$
w=-2-k R y y_{x} \cos \alpha \operatorname{tang} \frac{x}{2}
$$

espressione che assume il massimo valore

$$
\text { u } \therefore 2 \pi k \text { R } y y_{\mathrm{s}} 0.3003
$$

quando $\alpha=5 l^{\circ} .8$

Dalle [13] si ha pertanto la seguente relazione fra i numeri di Cassinis $f(Y), f(v)$ relativi alla stessa quota $H$, riferita rispettivamente alla sfera di raggio $R+H_{\varsigma}$ e alla sfera di raggio $R$ :

$$
f(Y)=f(y)+\varepsilon I I_{\mathrm{s}} \quad\left\{I_{\mathrm{s}}\right. \text { in chilometri }
$$

avendo posto

$\varepsilon=-2-k R y_{1}\left(\cos \gamma_{1} \operatorname{lang} \frac{\alpha_{1}}{2}-\cos \alpha_{2} \operatorname{tang} \frac{\alpha_{2}}{2}\right) \overline{y_{s}}$

e attribuito a $y_{\mathrm{s}}$ il valore $\bar{y}_{\mathrm{s}}$ corrispondente a $H_{\mathrm{s}}=+1 \mathrm{Km}$.

Velle Tavole fondamentali per la riduzione dei valori osservati 
della gracita $(*)$ e nelle tabelle relative alla ripartizione delle zone $L, M, N, O_{1}$, a fianco dei numeri f di Cassinis, calcolati per la sfera di ragrio $R=0.37 \mathrm{l} .2 \mathrm{Km}$, si trovano i valori $\varepsilon$ della correzione che tiene conto delleffetto secontario della quota di stazione.

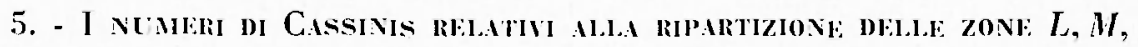
$N, O_{1}$ E ALLA ZONA $(A G)$ RIC NIONE DELLE PRINE ZONE.

1. - Con le formole sviluppate in precendenza, sono stati calcolati e in seguito raccolti nelle tabelle $V$. i mumeri di Cassinis relativi: alle sottozone che suddividono, per la valutazione delle sole riduzioni isostatiche, le zone L. V. Y.O, della sudulivisione di Ilavford, e alla zona $(A G$, che comprente le zone immediatamente prosime alla stazione.

Le tabelle, che costituiscono un completamento delle Tavole fondamentali, non hamno bisogno di essere illustrate poiché tanto i simboli che le uniti di misura, le costanti impiegate e la disposizione jei valori, sono wli stessi di quelli aloperati nelle dette tavole fondamentali. Va soltanto notato ehe a diferenza di quest'ultime, l'argomento quota varia nell'intervallo più ristretto: - $5000 \mathrm{~m}$. $70000 \mathrm{~m}$, nel quale sono comprese le quote che interessano ordinariamente le sole riduzioni isostatiche. Inoltre le variazioni tabulate dei numeri di Cassinis corrispondono sempre a variazioni di $-100 \mathrm{~m}$ della quota.

Ricordiamo ancora che per definizione il numero $f$ di Cals-inis: relativo ad una zona limitata dai valori angolari $\oint_{1}<$ () $_{2}$ c compresa fra la quota zero e la quota $H$. riferite alla sfera di ragrio $R=6$.37 1.2 $\mathrm{Km}$. viene espresso. in uniti di $1.10^{-2}$ gal, dalle differenze:

$$
\begin{aligned}
& f(x)=F\left(x_{1}, y\right)-F\left(x_{2}, y\right) \quad \text { se la quota II is positiva } \\
& f(y)=F\left(x_{2}, y\right)-F\left(x_{1}, y\right)
\end{aligned}
$$

dove: $\mathrm{x}=\sin \frac{\oint}{2} ; y=\frac{H}{R}$ e la funzione $\mathrm{F}(\mathrm{x}, \mathrm{y})$ rappresenta la componente verticale dellattrazione esercitata da ma calotta sferica di ampieza angolare $\varnothing$, di spessore $I I$ e di densita uno.

Sicché per ottenere l'attrazione. cambiata di semno. esercitata dalla zona compresa tra le quote $\mathrm{H}_{1}<\mathrm{H}_{2}$ sulla massa unitaria posta nel punto $S$, di quota zero. del sto asse. c cioc la riluzione $\triangle$ I da appor-

(*) G. Cassivis, P. Dom: S. BatLmis, Tavole jondamentali per la riduzione dei valori osservati della gratita. R. Commissione geodetica italiana. Pubblicazioni: Nuova serie, 11. I3, Pavia, 1937. 
tare al valore osservato in $S$ della gravita occorre fare la differenza: $f\left(y_{2}\right)-I\left(y_{1}\right)$ per poi moltiplicare per la densità della materia costituente la zona:

$$
\Delta f=\delta\left[f\left(y_{2}\right)-f\left(y_{1}\right]\right.
$$

Nel caso generale di una stazione di quota $H_{s}$ si ha

$$
\Delta f=\delta\left[f\left(y_{2}\right)-f\left(y_{1}\right)+H_{s}\left(\varepsilon_{2}-\varepsilon_{1}\right)\right]
$$

nella quale come si è visto nel paragrafo precedente, $\varepsilon_{2}, \varepsilon_{1}$ rawbre-entano le correzioni dovute all'effetto secondario dell'altezza $\mathrm{HI}_{*}$ della stazione, corrispondenti alle quote $H_{z}, H_{1}$ e quando $H_{s}=+1 \mathrm{~km}$.

Pisa - Istituto di Geodesia e Topografia dell Università - Feb. braio 1954.

\section{RIASSUNTO}

Vengono esposti $i$ criteri che hamno presieduto alla ripartizione in sottozone, per il calcolo delle riduzioni isostatiche, delle zone notevolmente ampie L. M, N, O della suddivisione di Hayford, $e$ procedimenti di calcolo seguiti nella determinazione dei numeri di Cassinis relativi alla nuova suddivisione.

\section{SLMMARY}

The criteria which have been adopted for the division in subzo. nes, for the calculation of the isostatic reductions, of the very large zones L. M. N, $\mathrm{O}_{1}$ of the subdivision of Hayford. and the calculation procedures followed in the determination of the number of Cassinis for the new subdivision, are given. 
TABELLA 5 ZONA (AG) $d_{1}=0$ $\left.i_{2}, \cdots 3521\right) m$

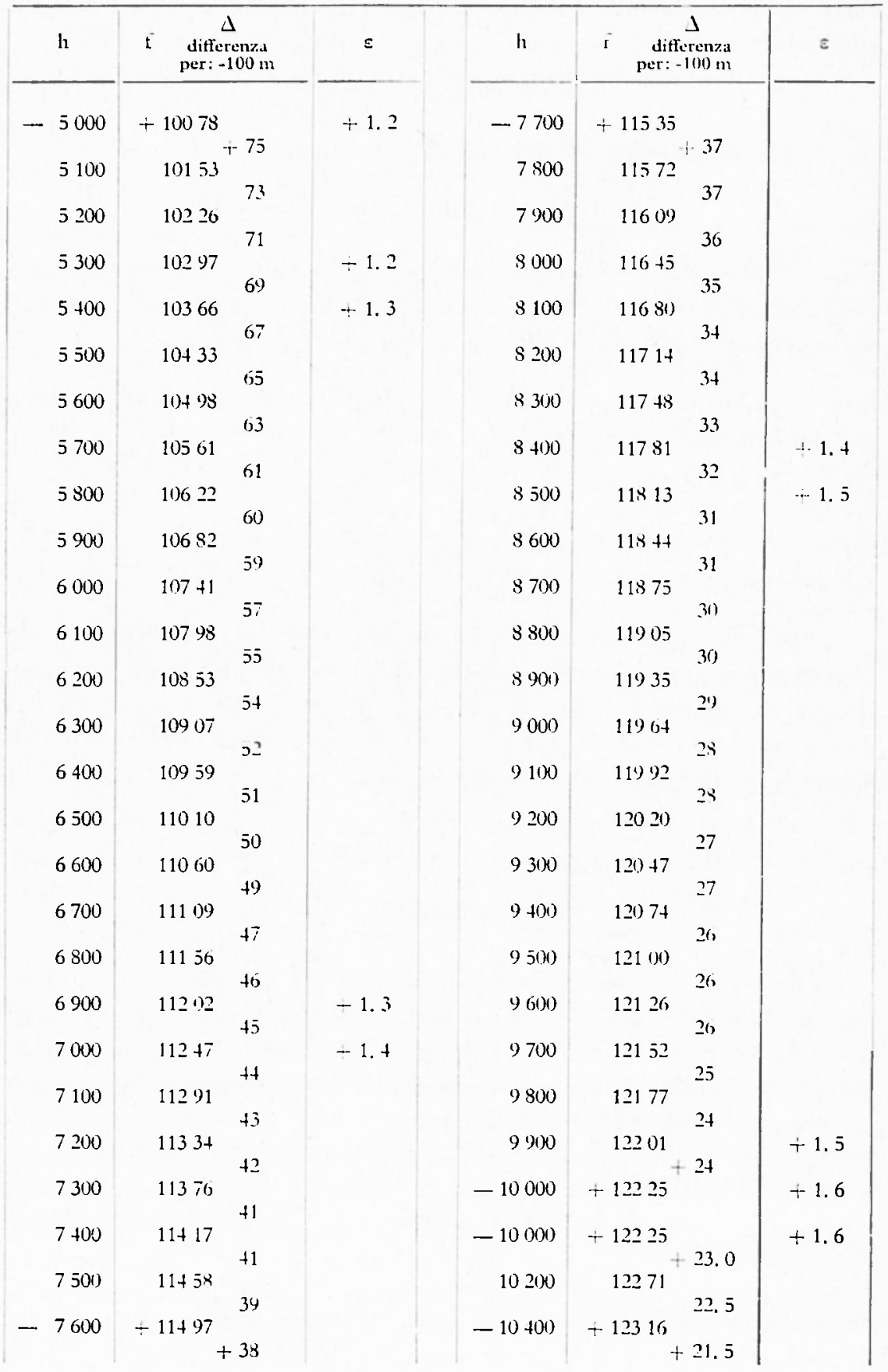


Contimu Tab. Y.

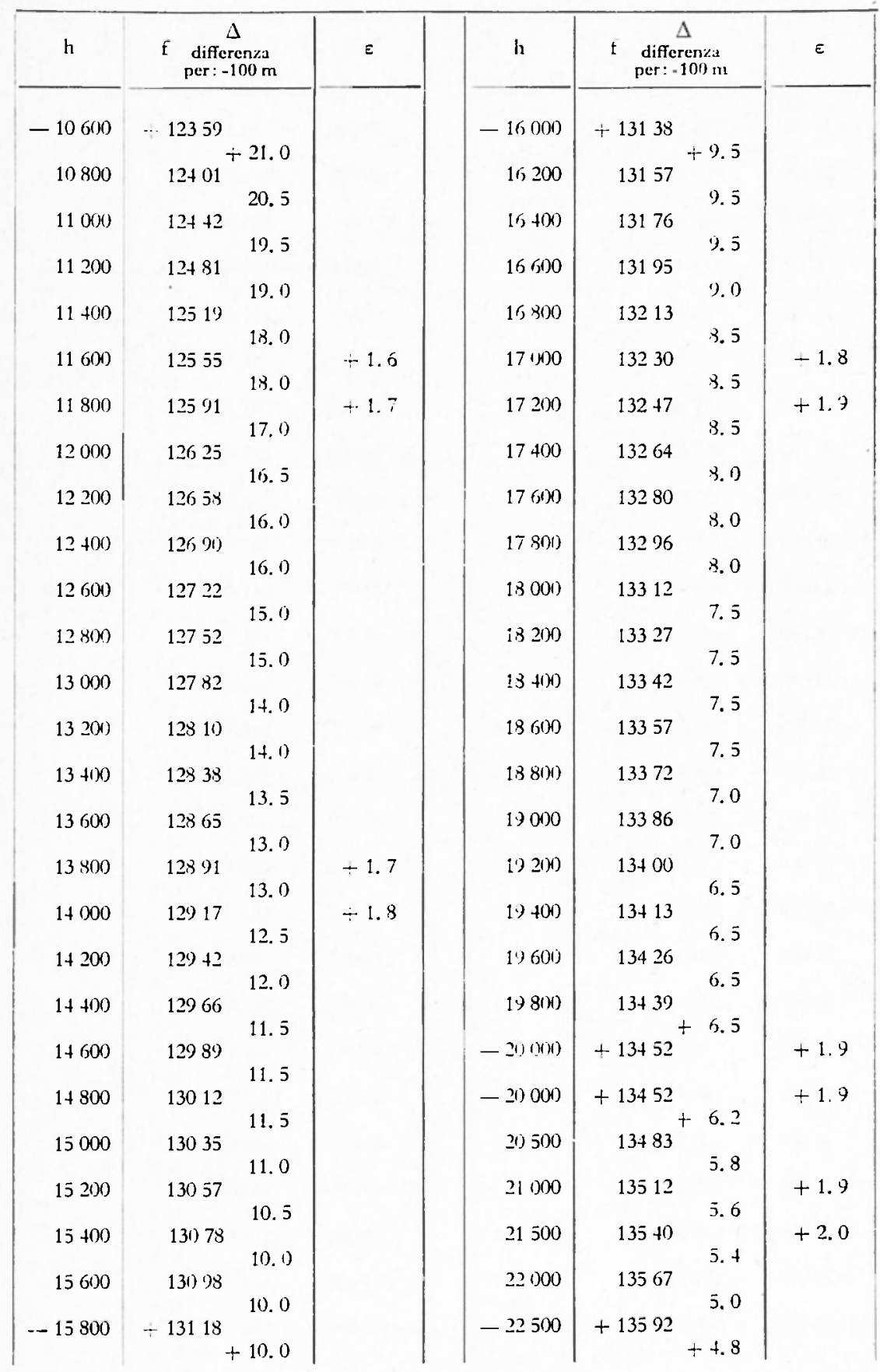


Conmina Tab. I:

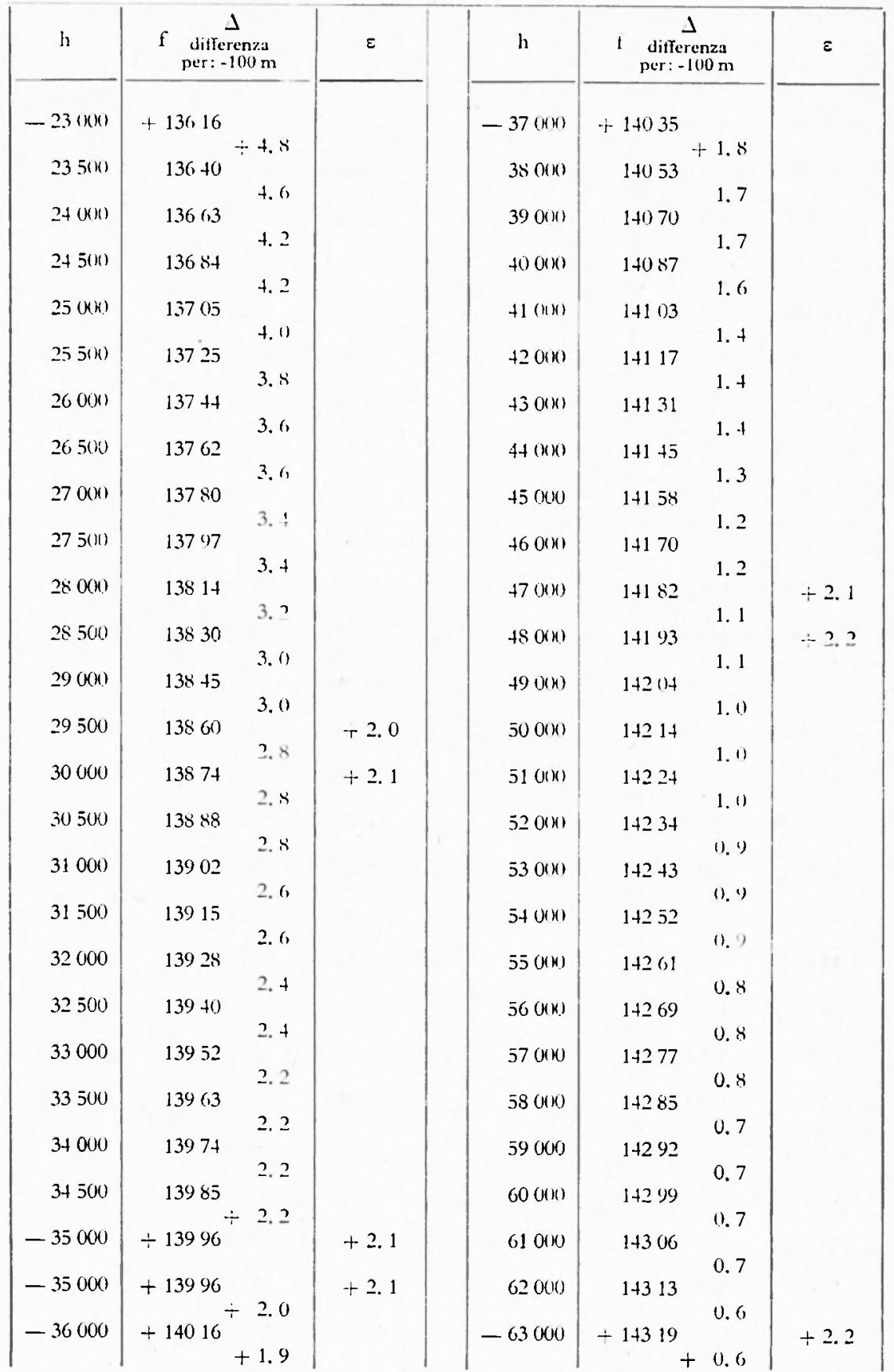


Comima Tab. $\%$.

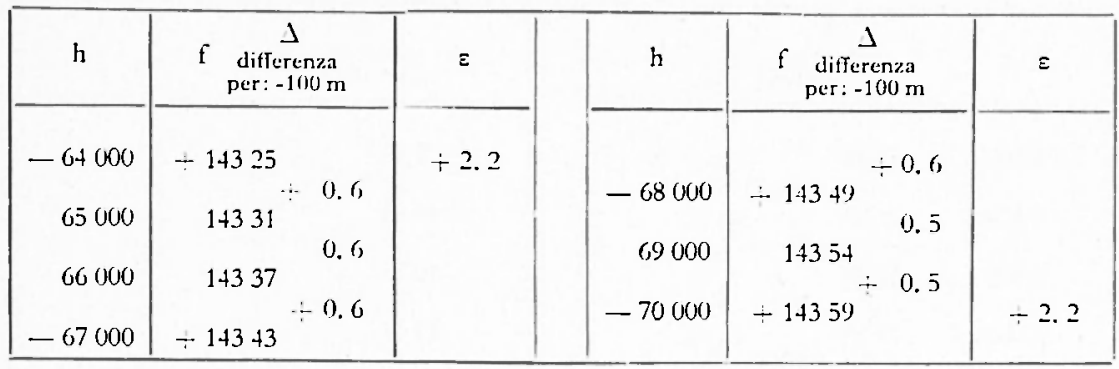

TABELLA V.

SOITOZONE: L'

$\mathrm{L}^{\prime \prime}$

$\mathrm{d}_{1}=18800 \mathrm{~m}$

$\mathrm{d}_{2}=23920$

$d_{1}=23920 \mathrm{~m}$

$d_{2}=28800$

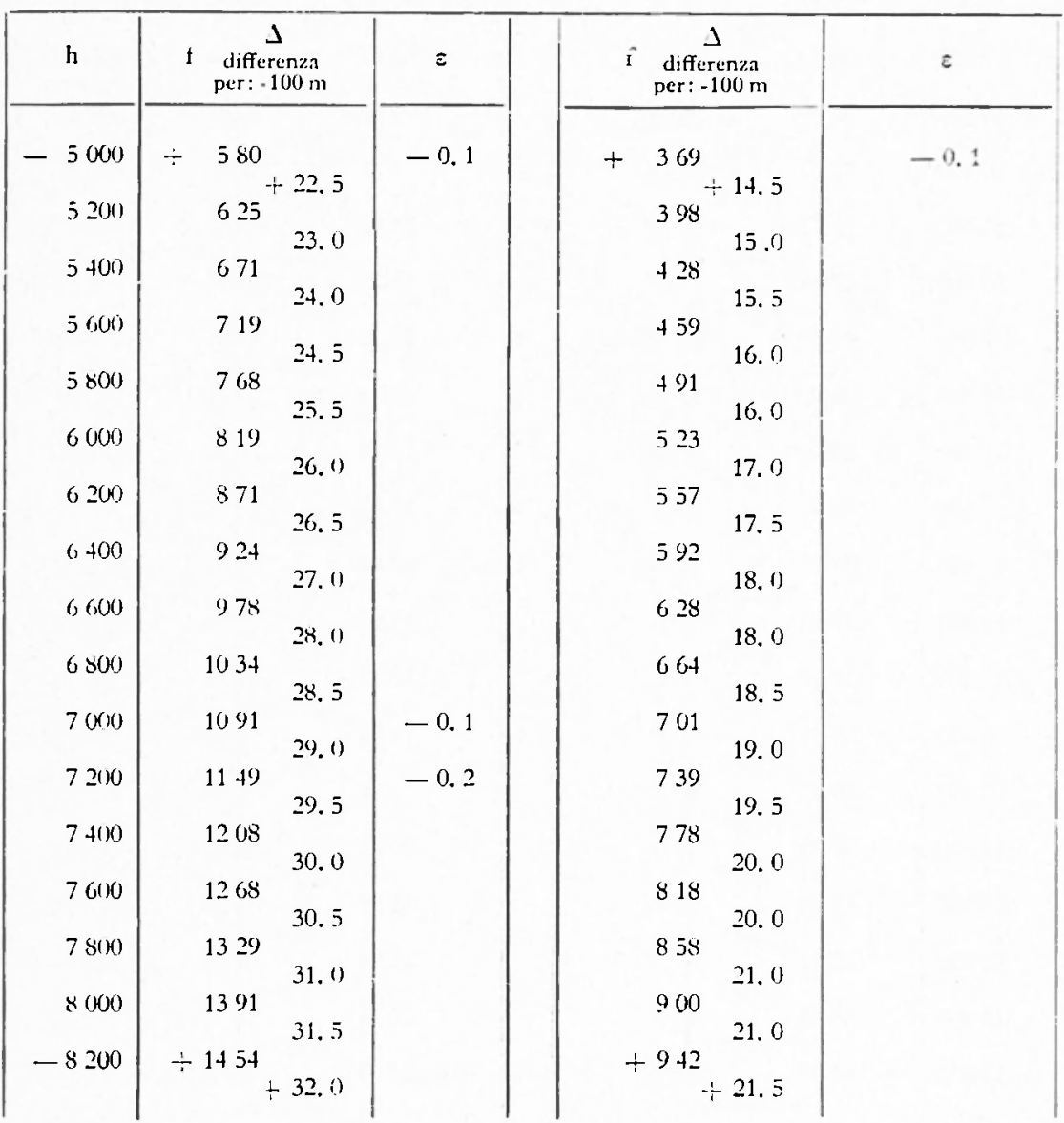


Continna Tab. IV.

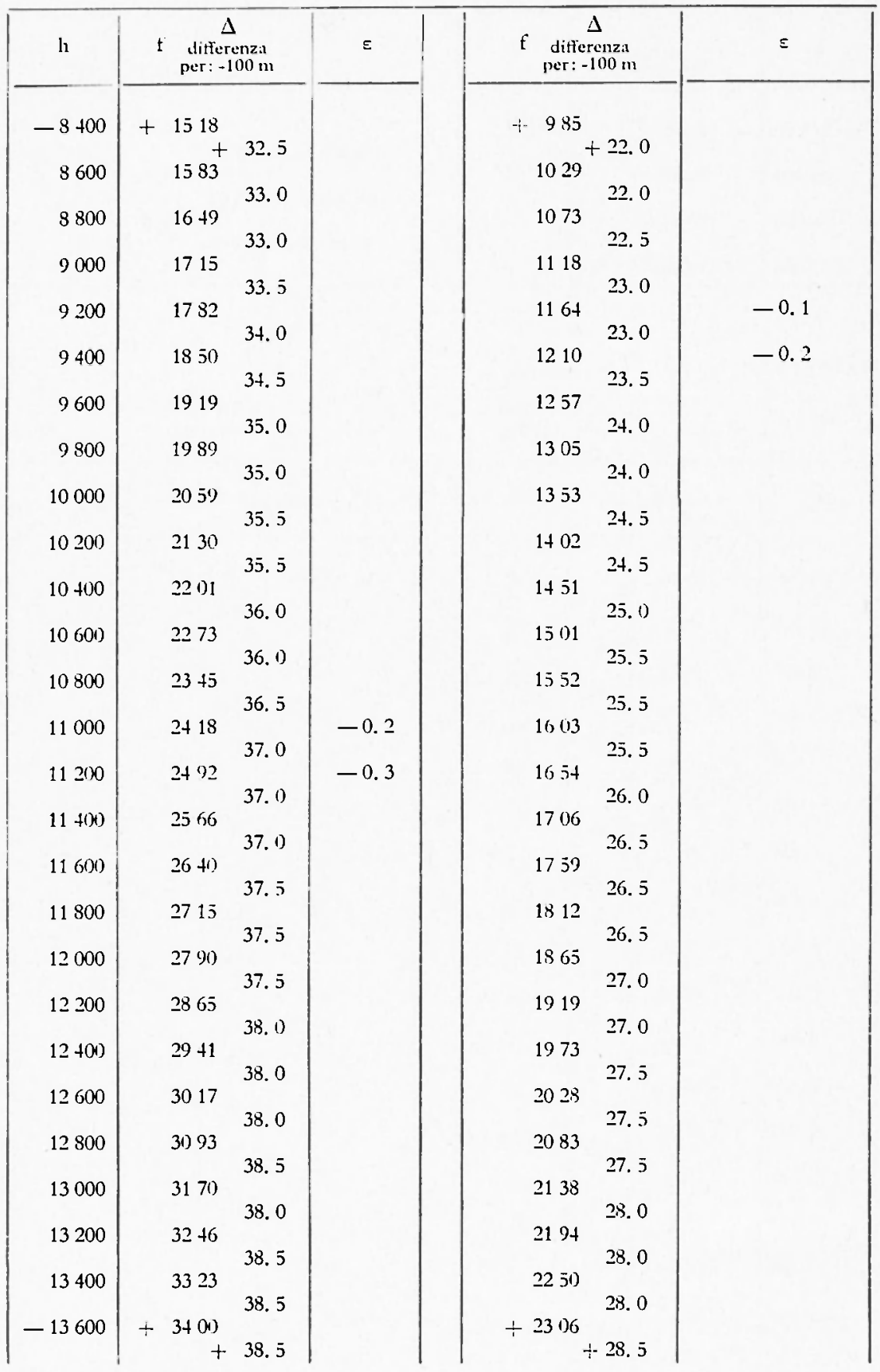


Continua Tab. \%.

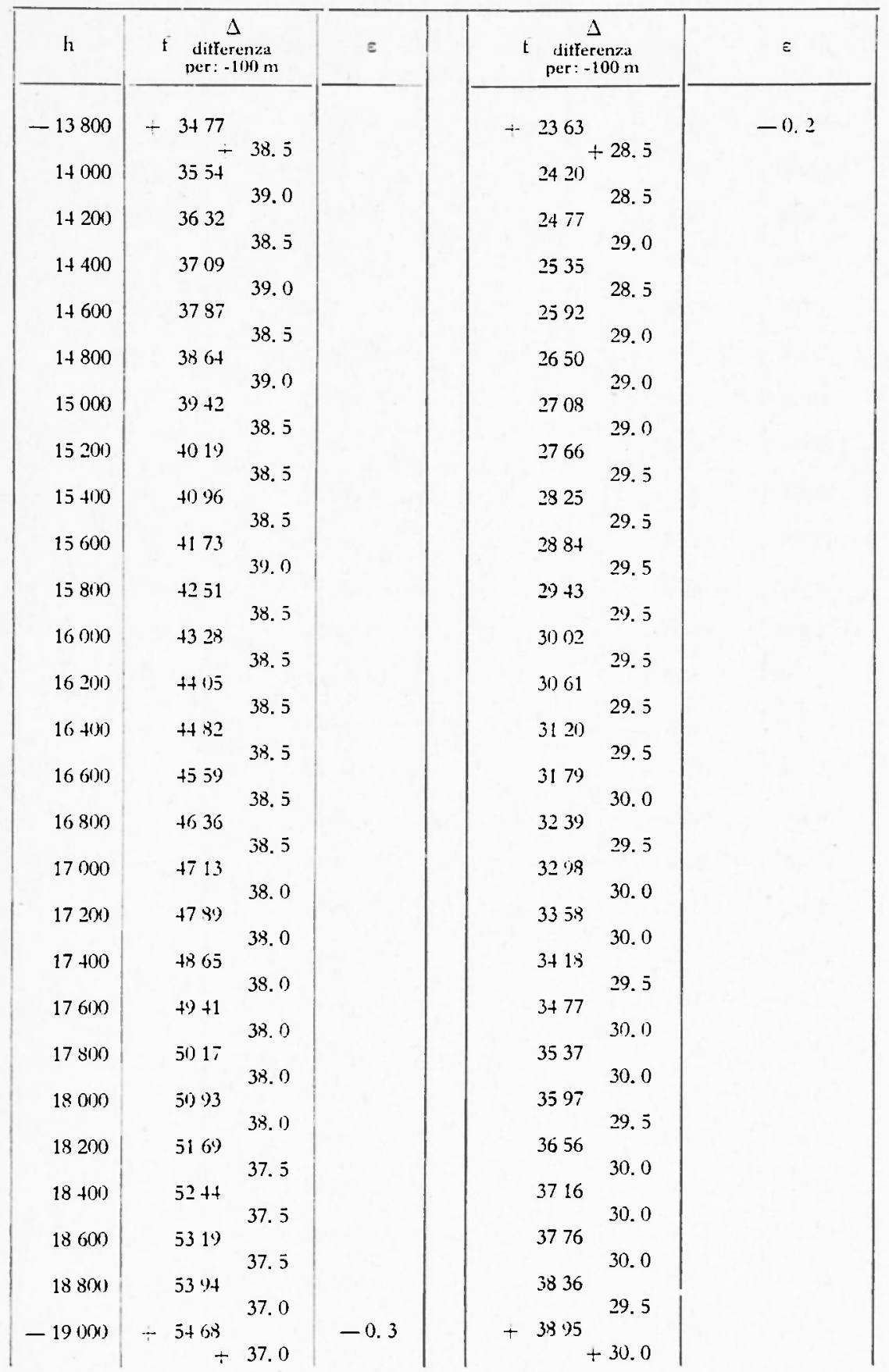


Continur Tab. I:

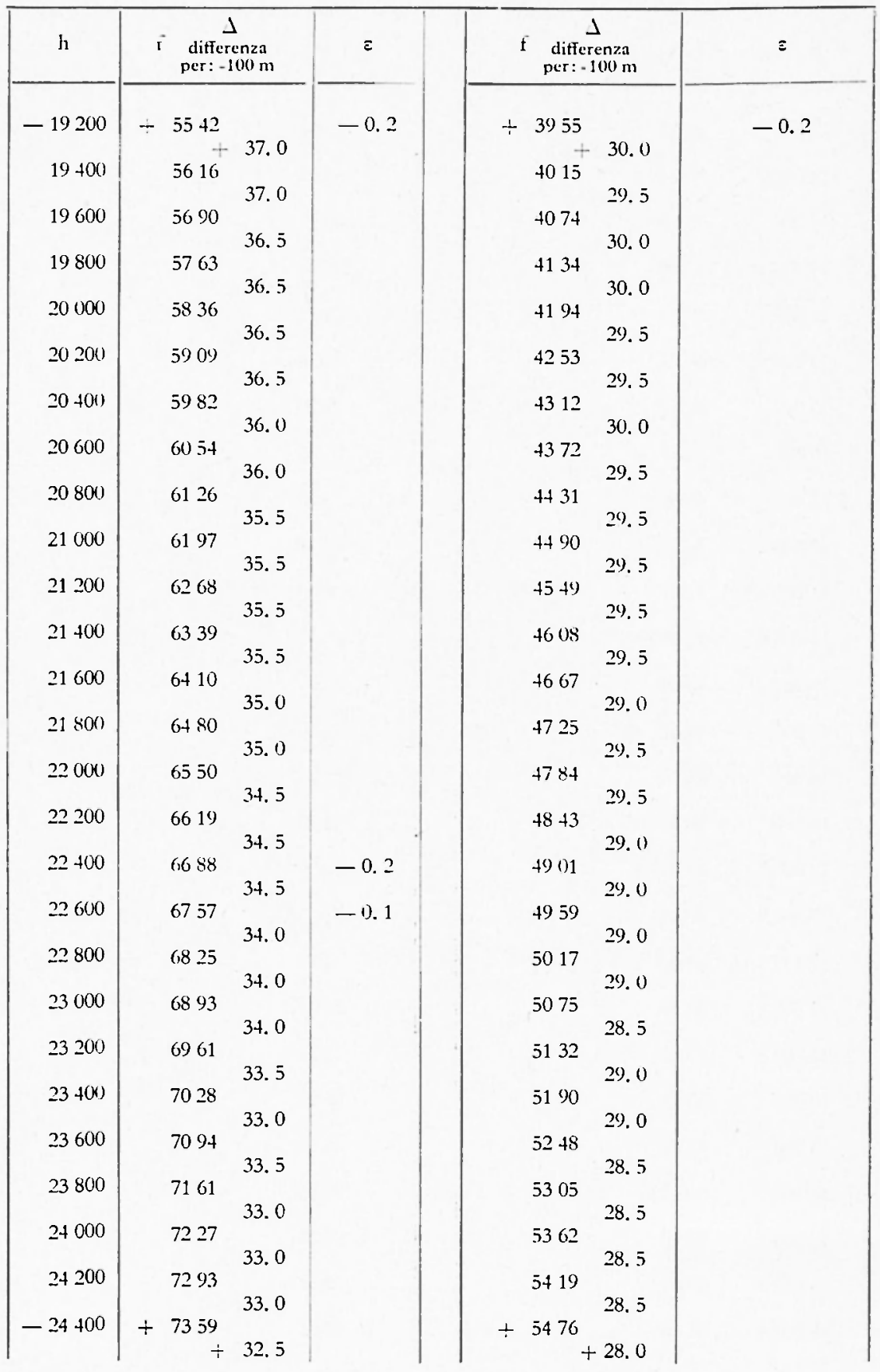


Continma Tab. V.

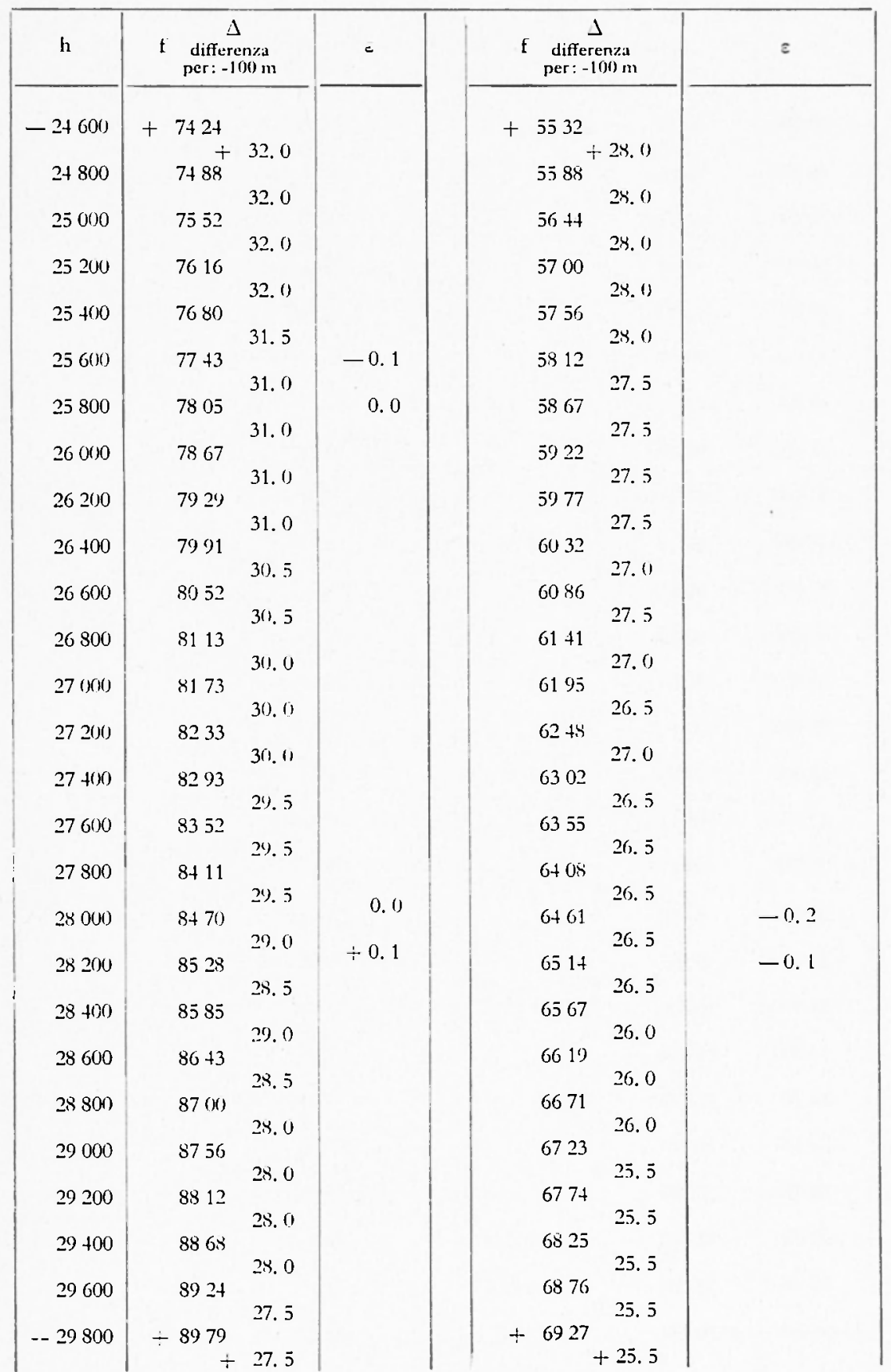


Combinua Tab. I'.

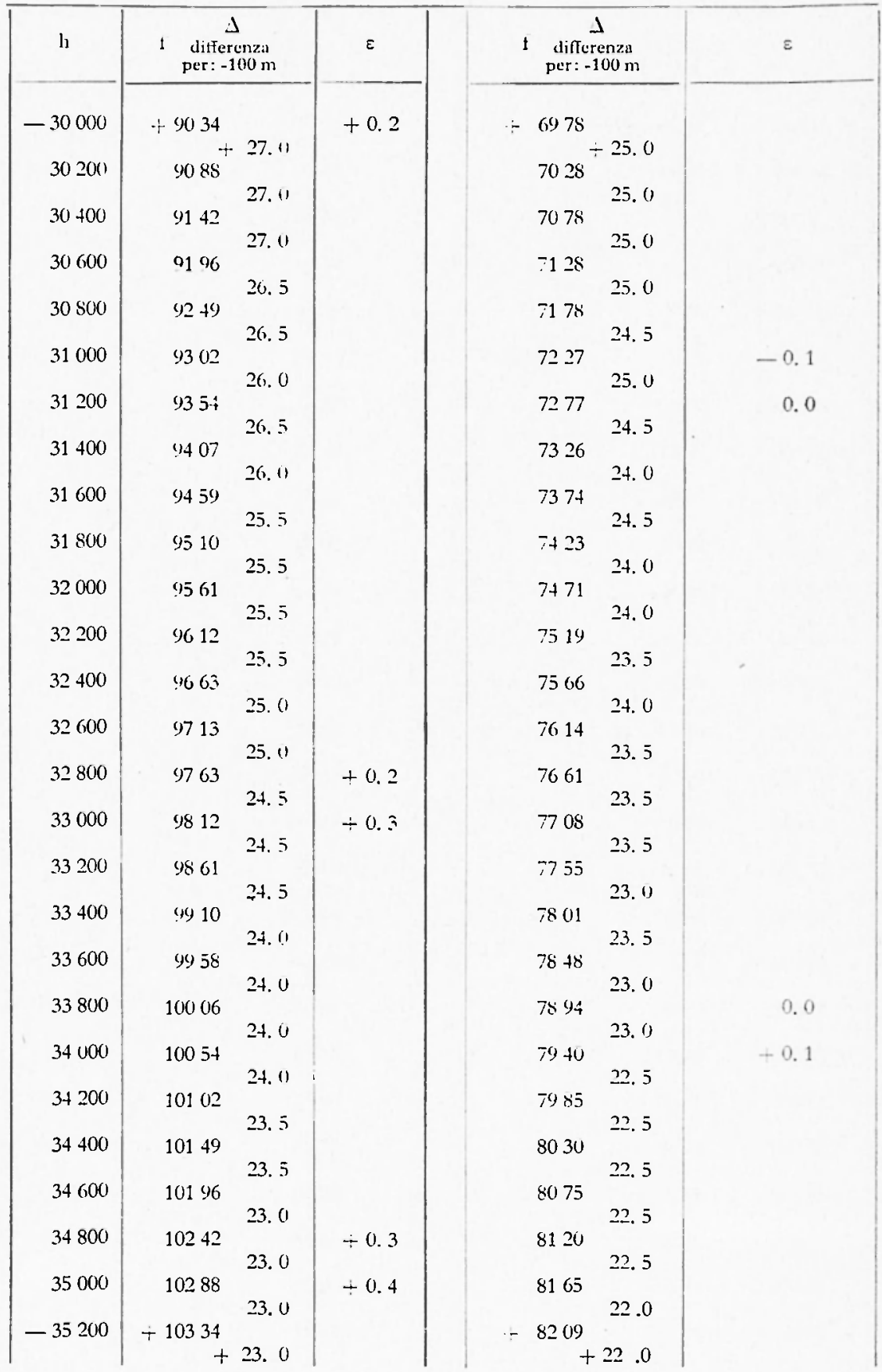


Cominua Tab. V.

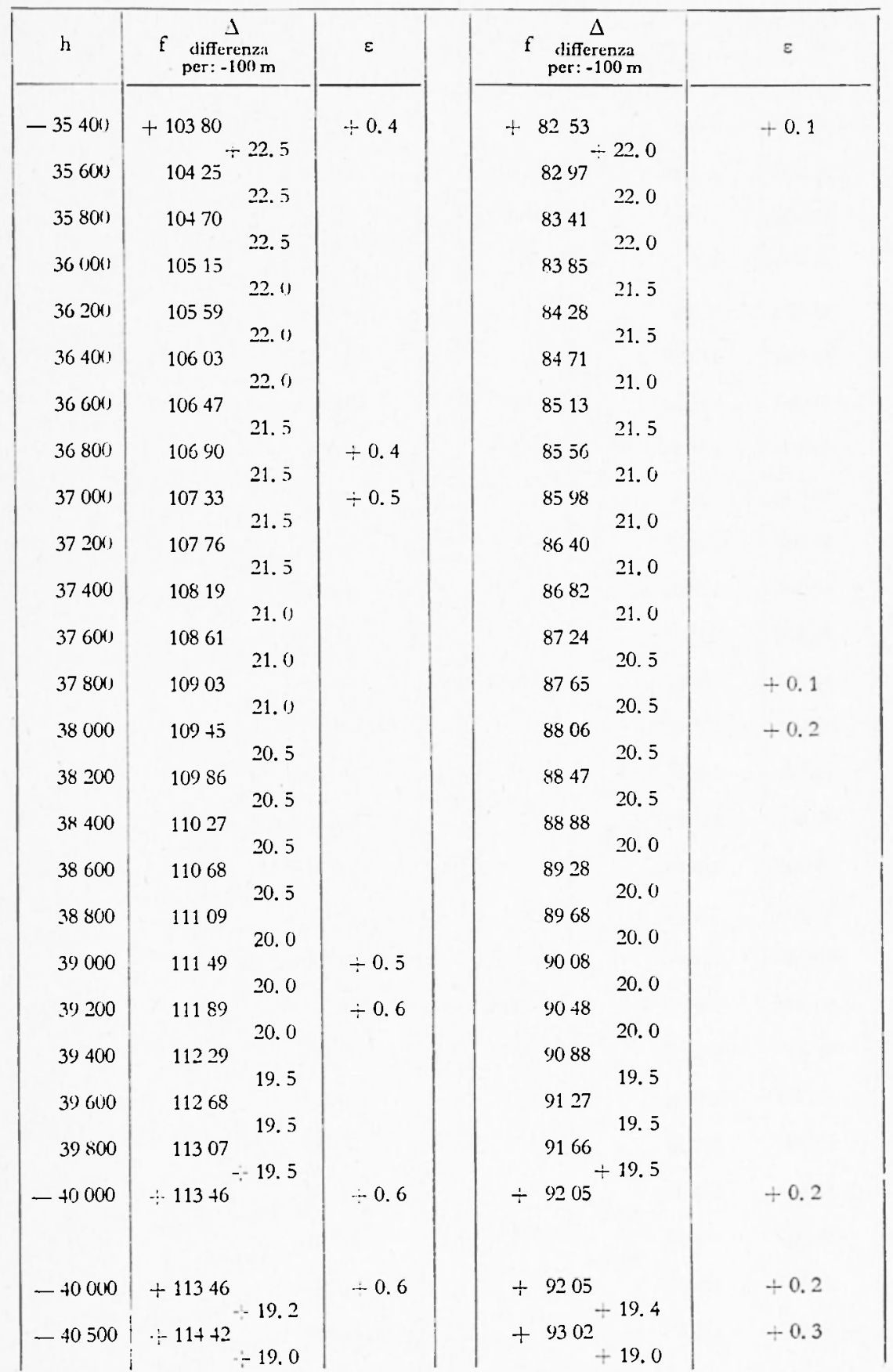


Comrimu Tal. I:

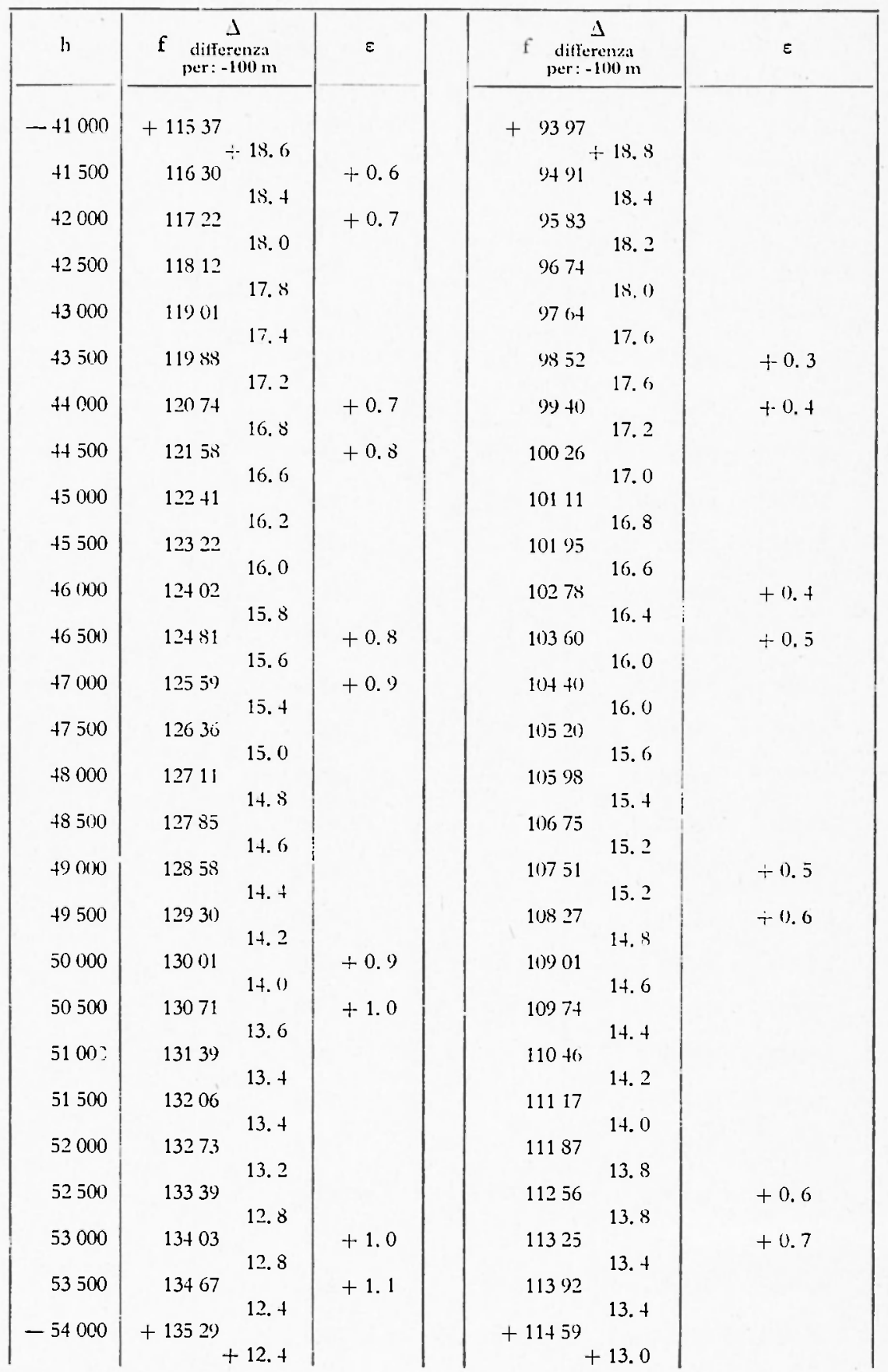


Continua Tab. V.

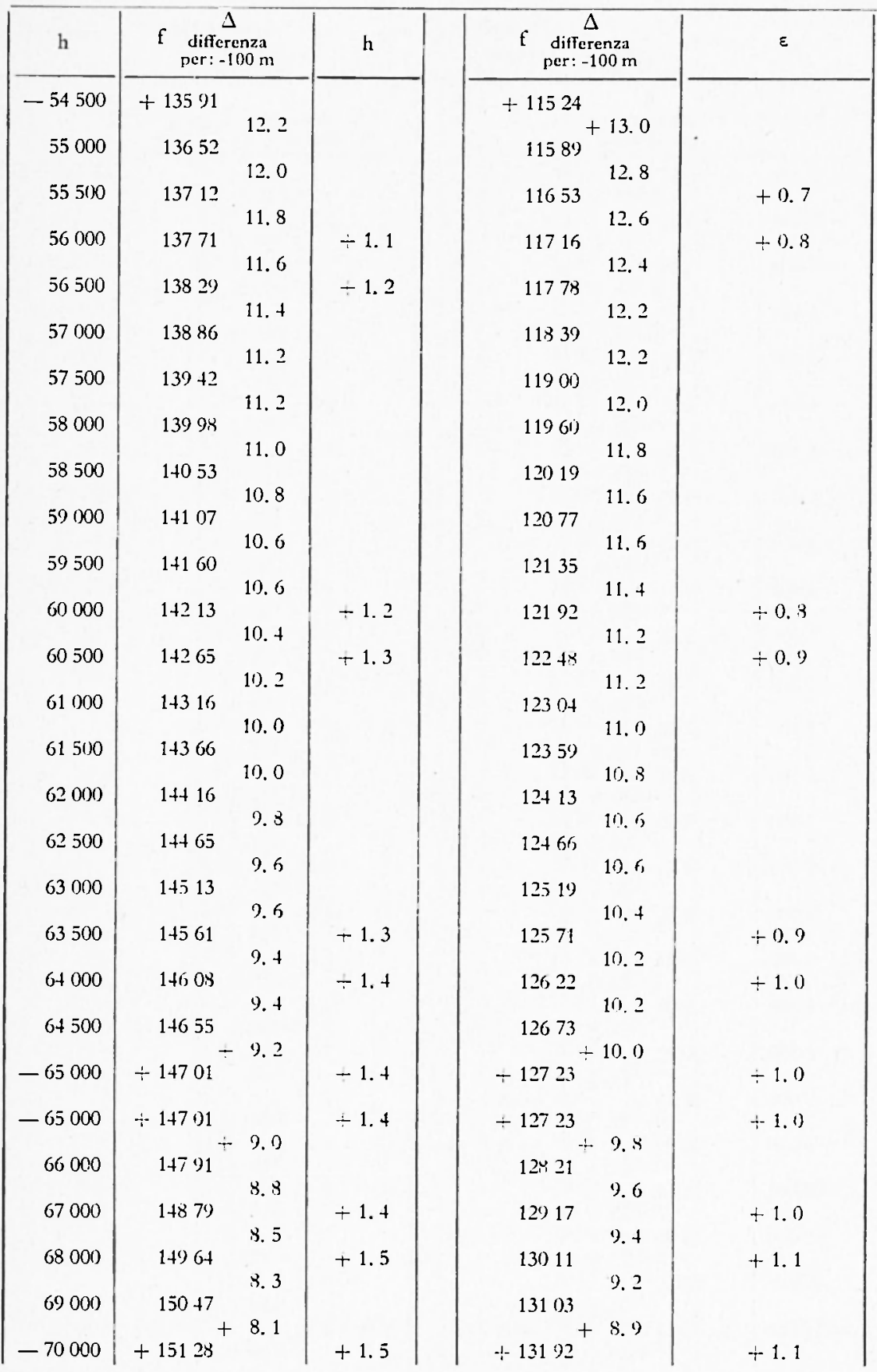


TABELLA V SOTTOZONE: $\mathrm{M}^{\prime}$ $\mathrm{M}^{\prime \prime}$

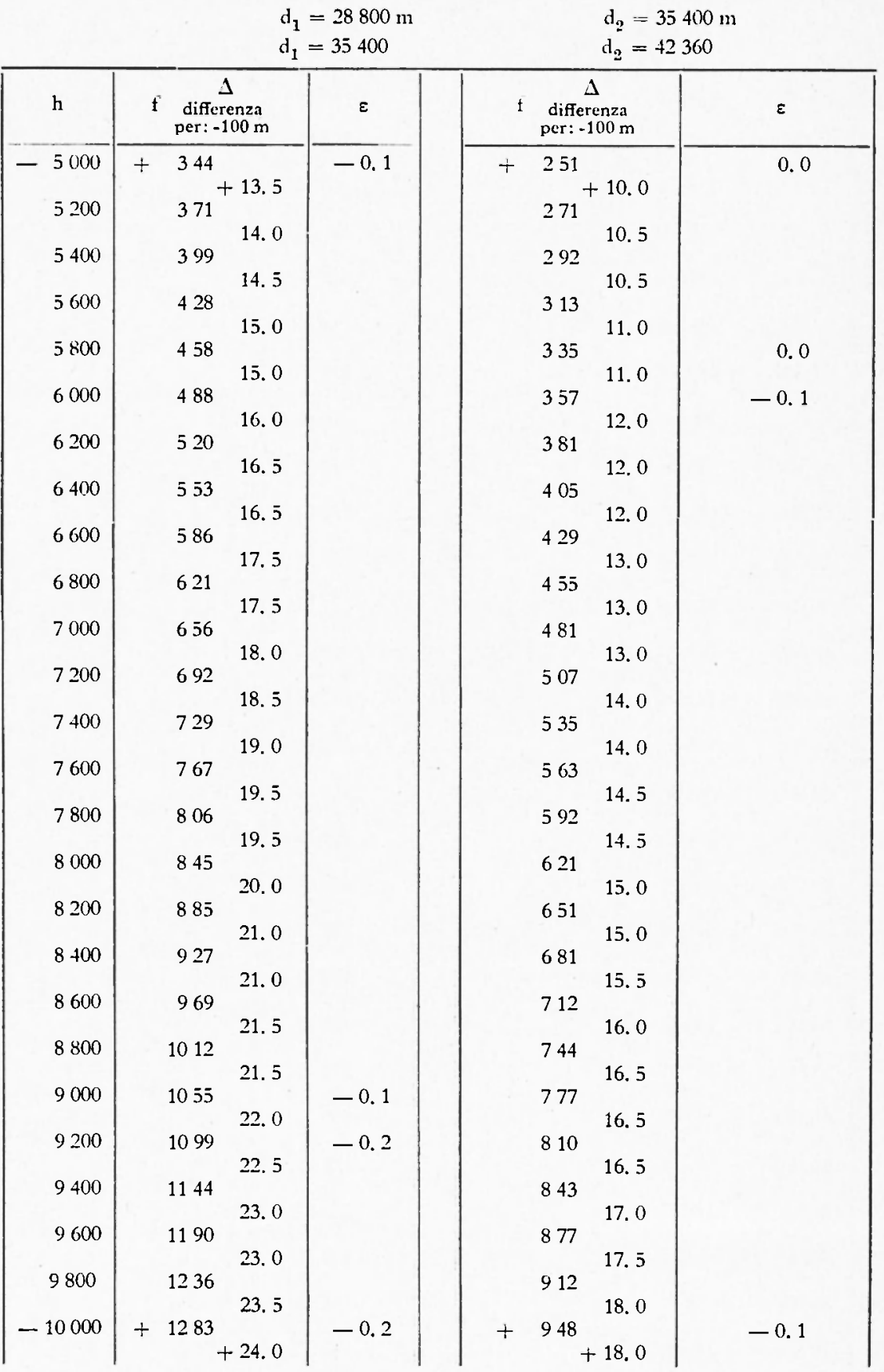


Continua Tab. V.

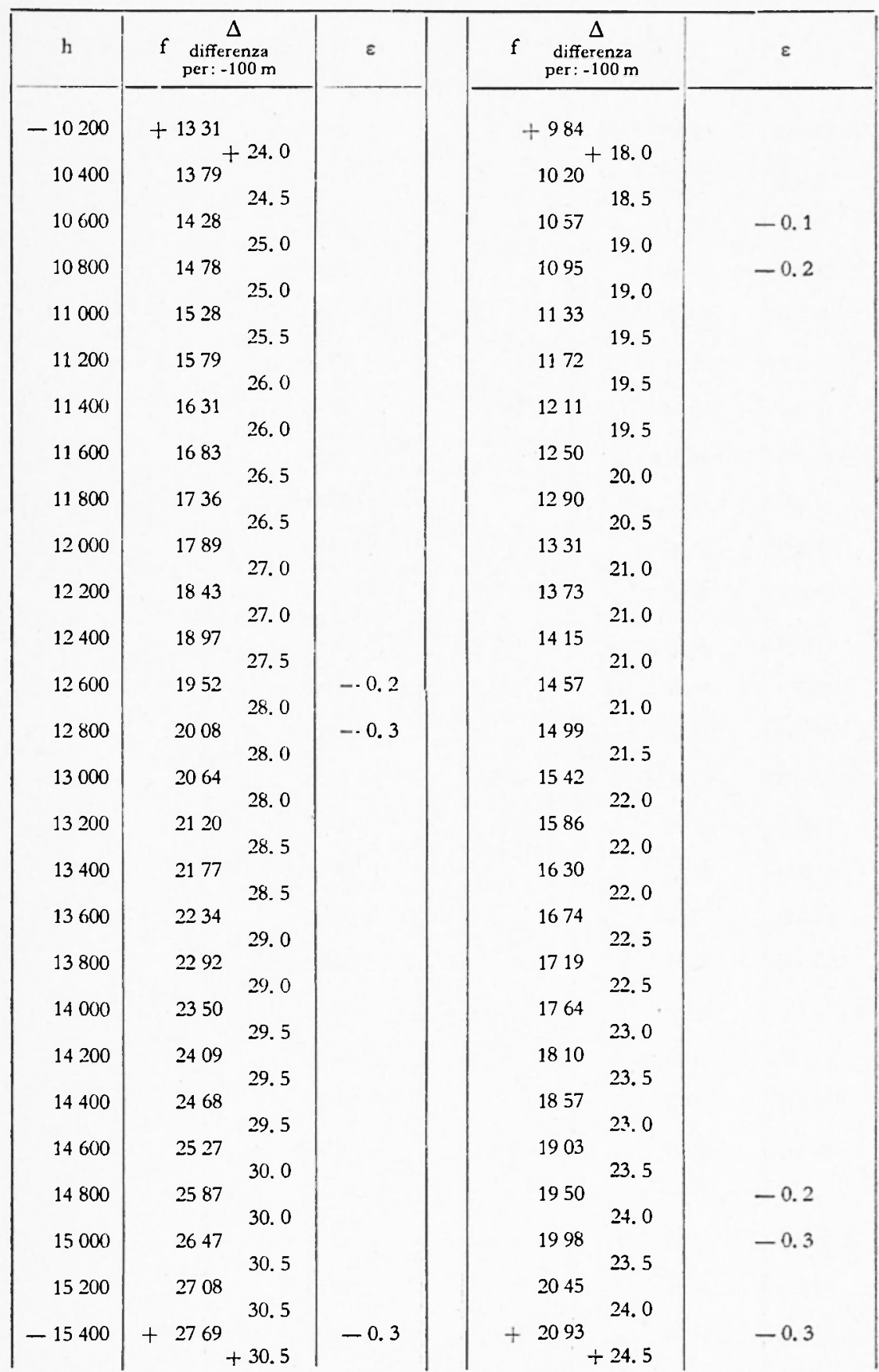


Cuntinua Tab. V.

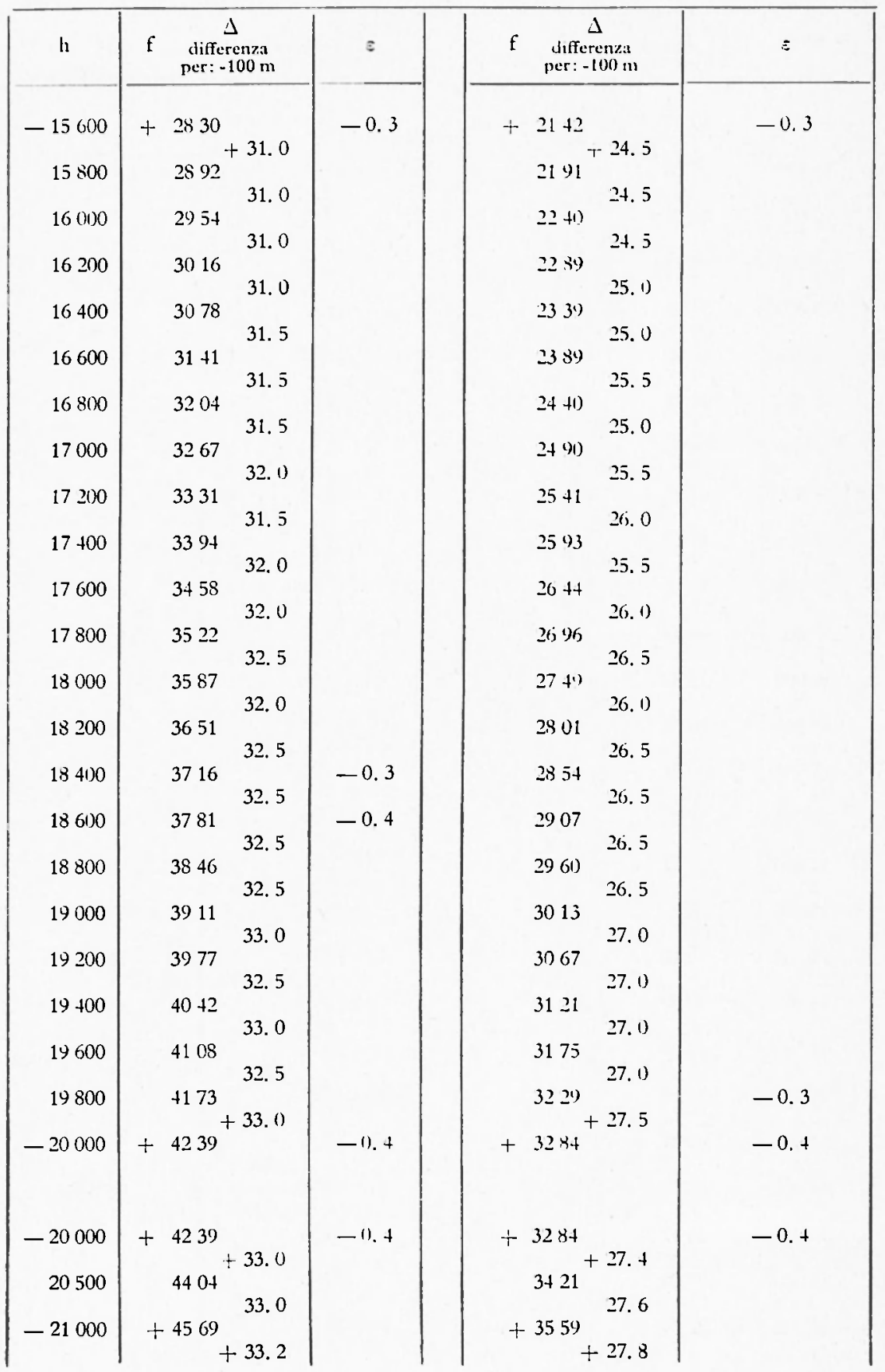


Continua Tab. V.

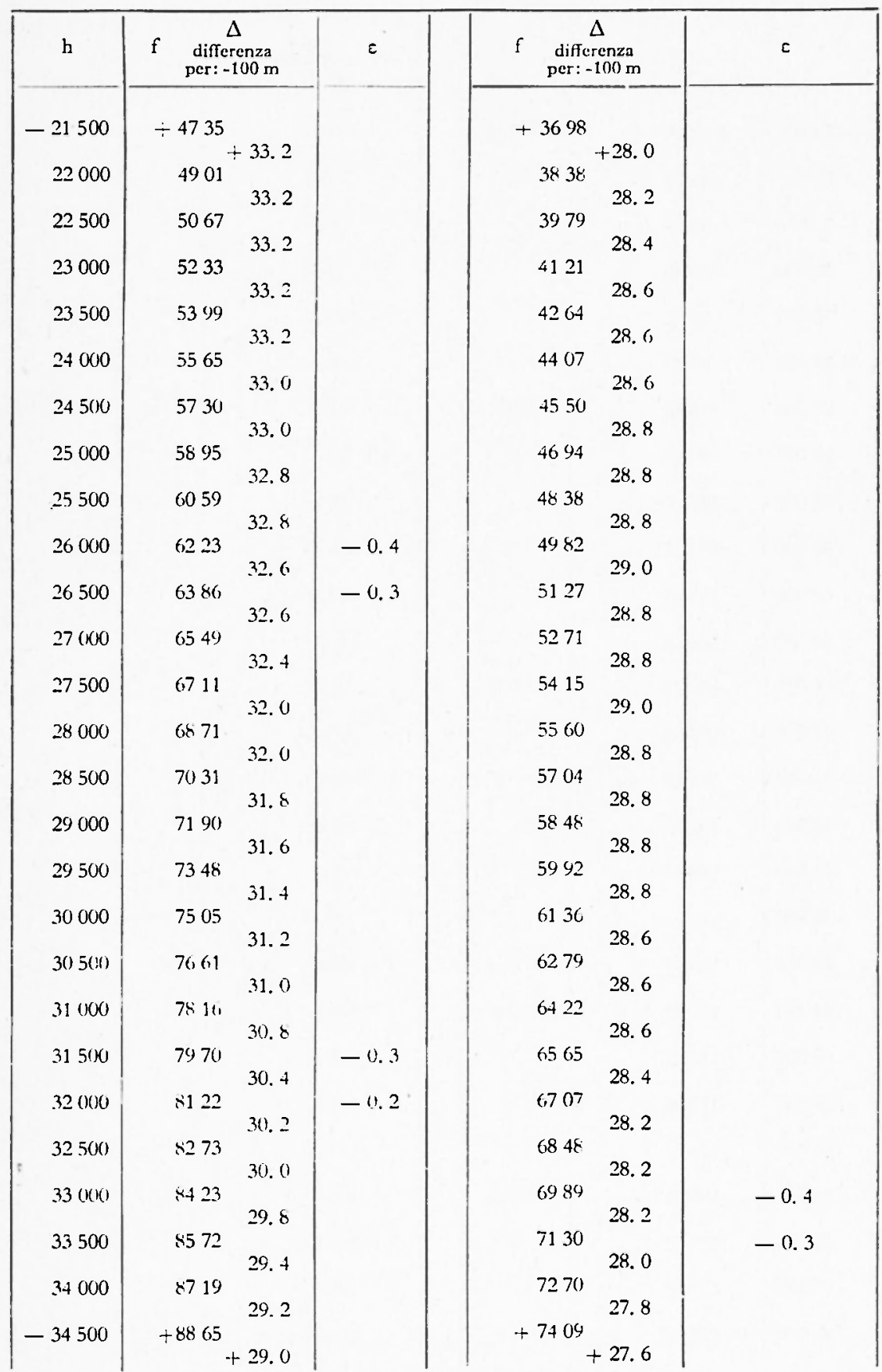


Continua Tab. I:

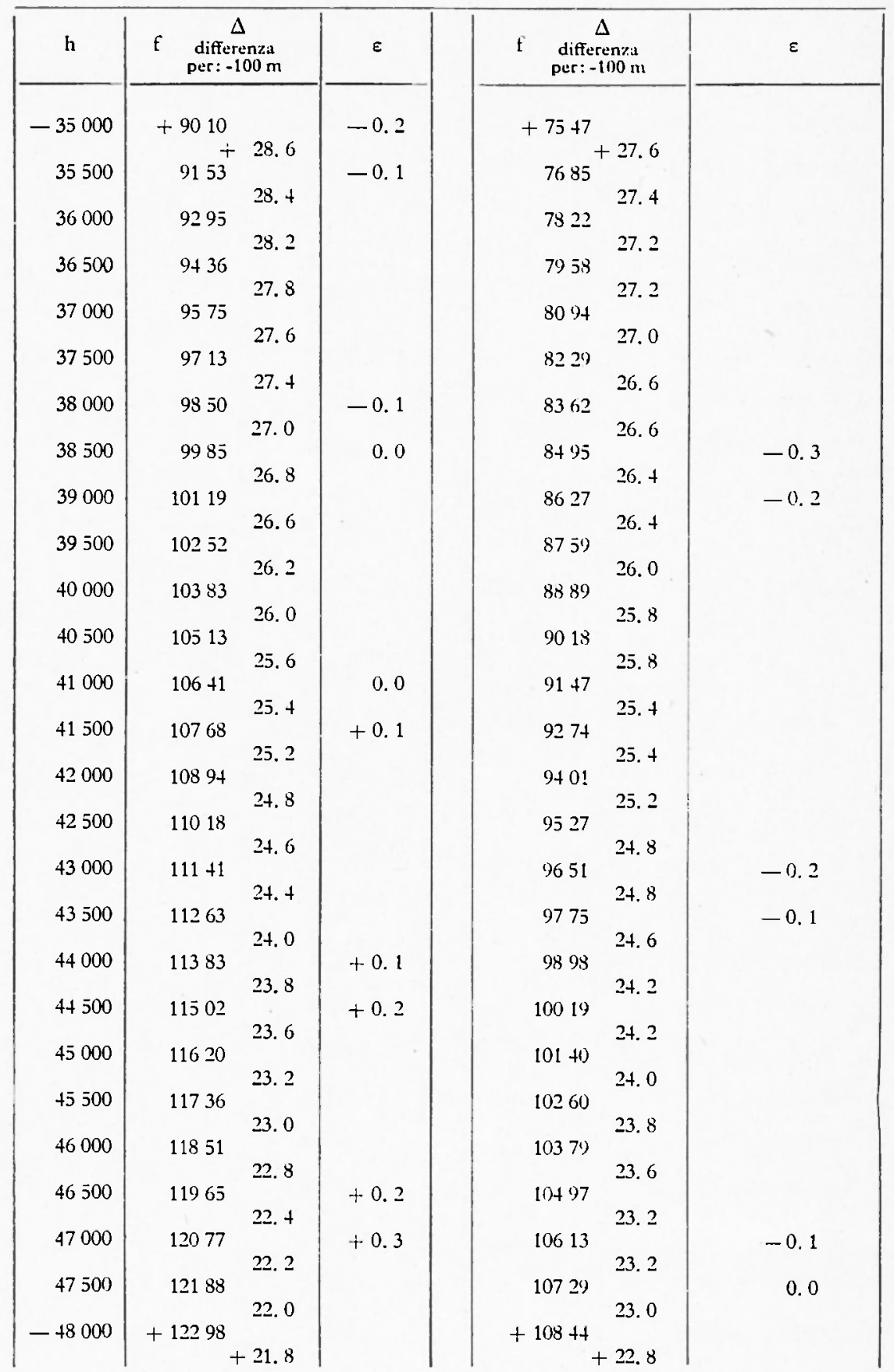


Continua Tab. V.

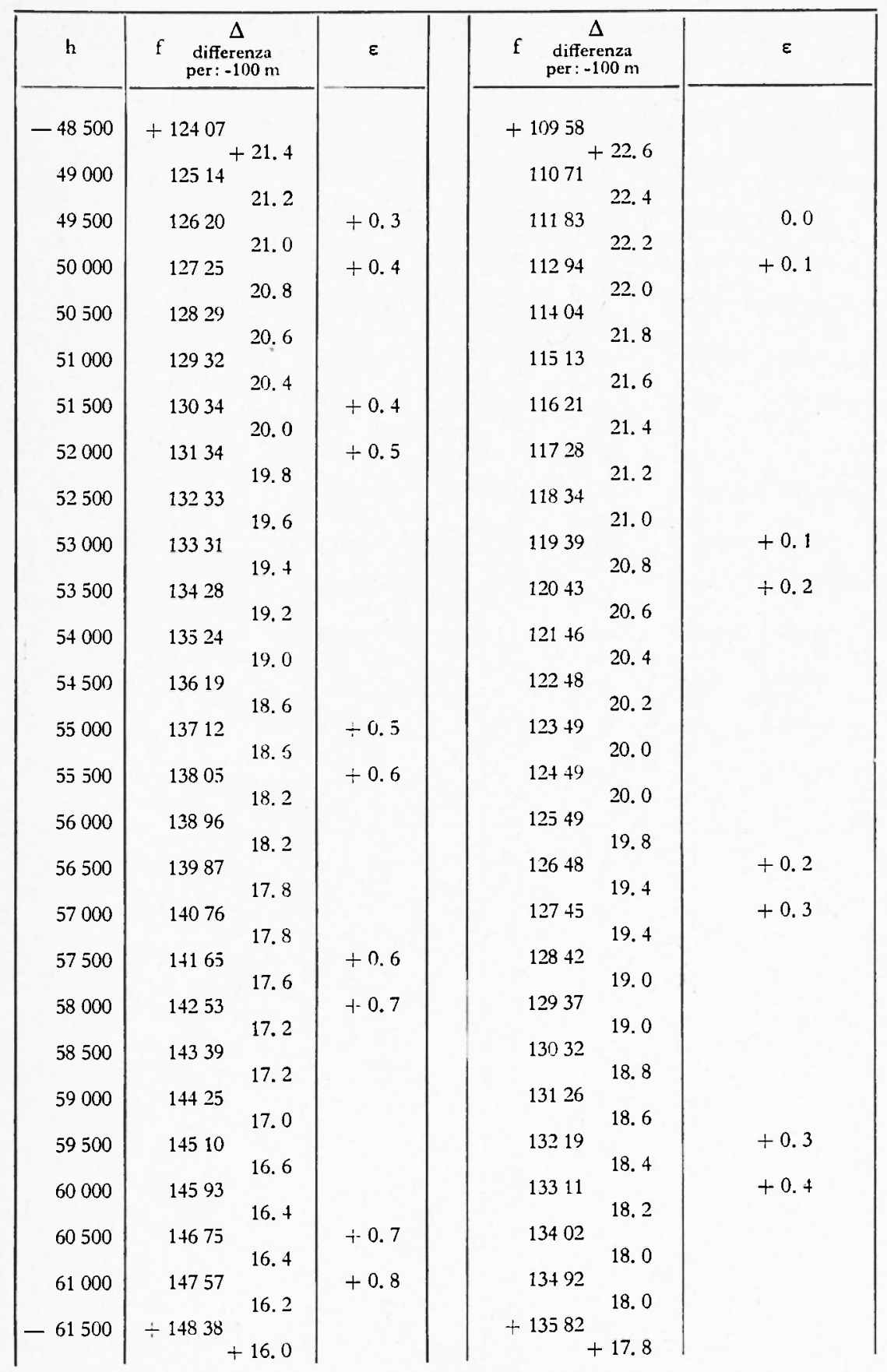


Continus Tob. I'.

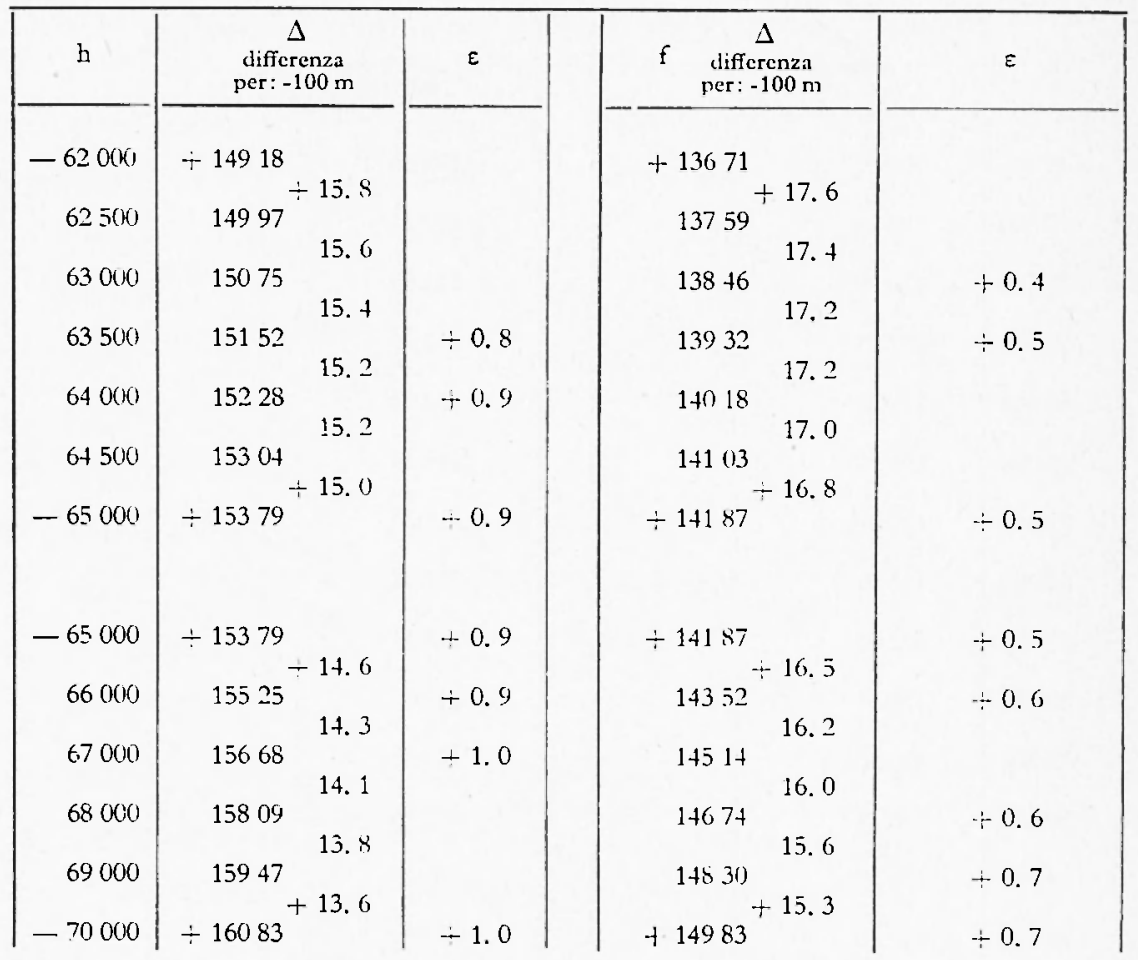


TABELLA V SOTTOZONE: $\mathrm{M}^{\prime \prime \prime}$ $M^{\prime \prime \prime \prime}$

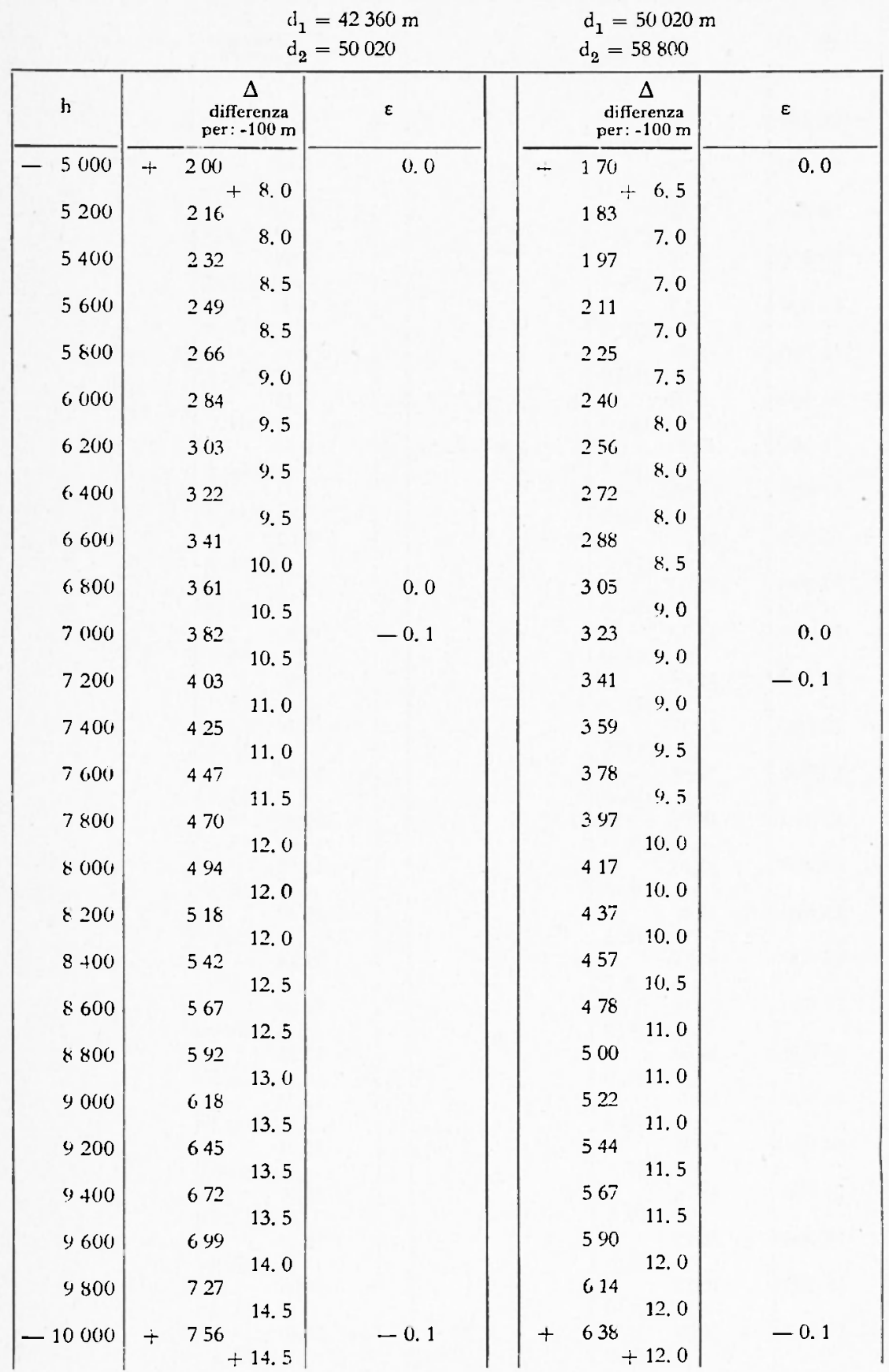


Contima Tab. V

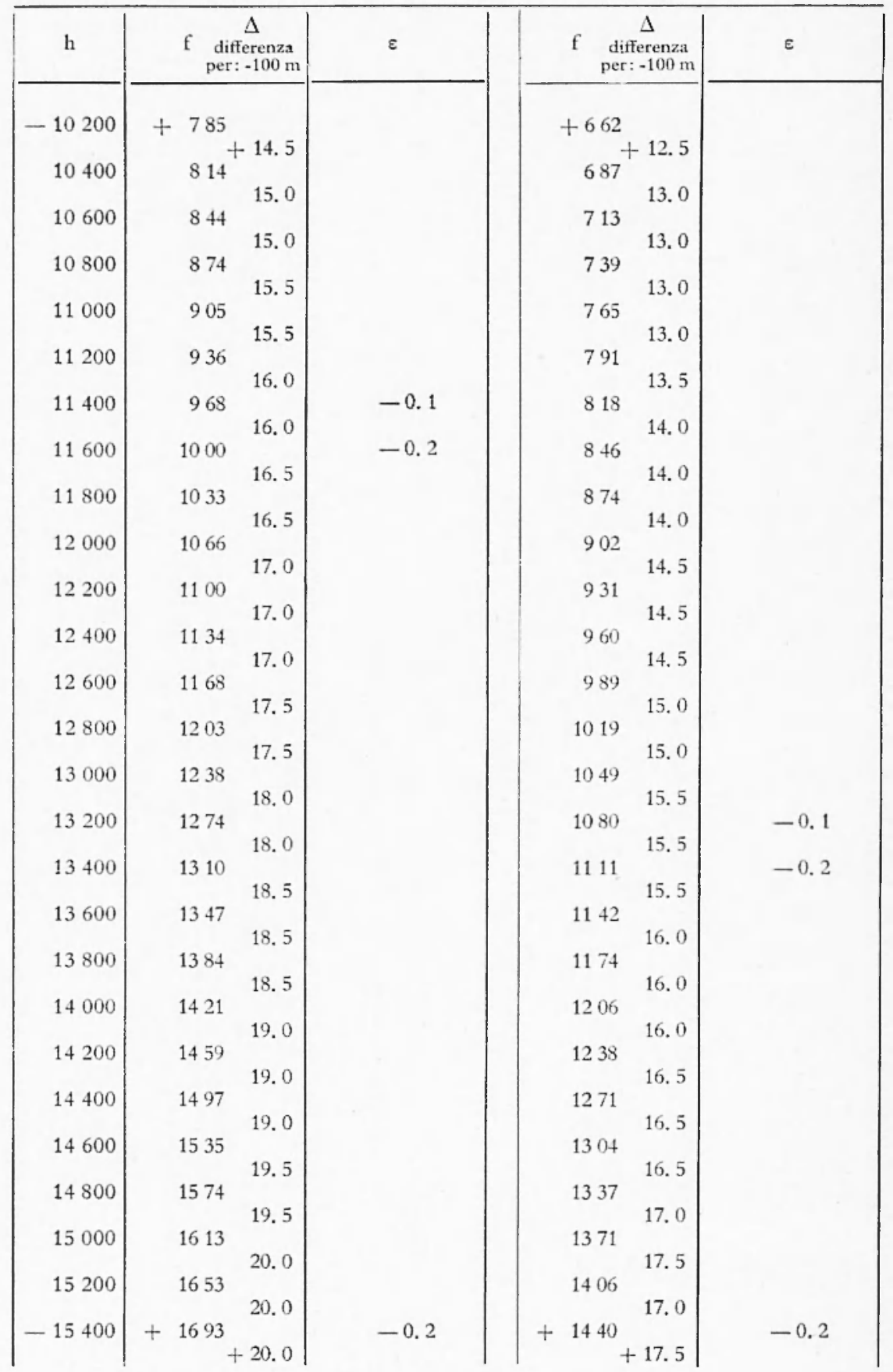


Continta Tab. $\Gamma$.

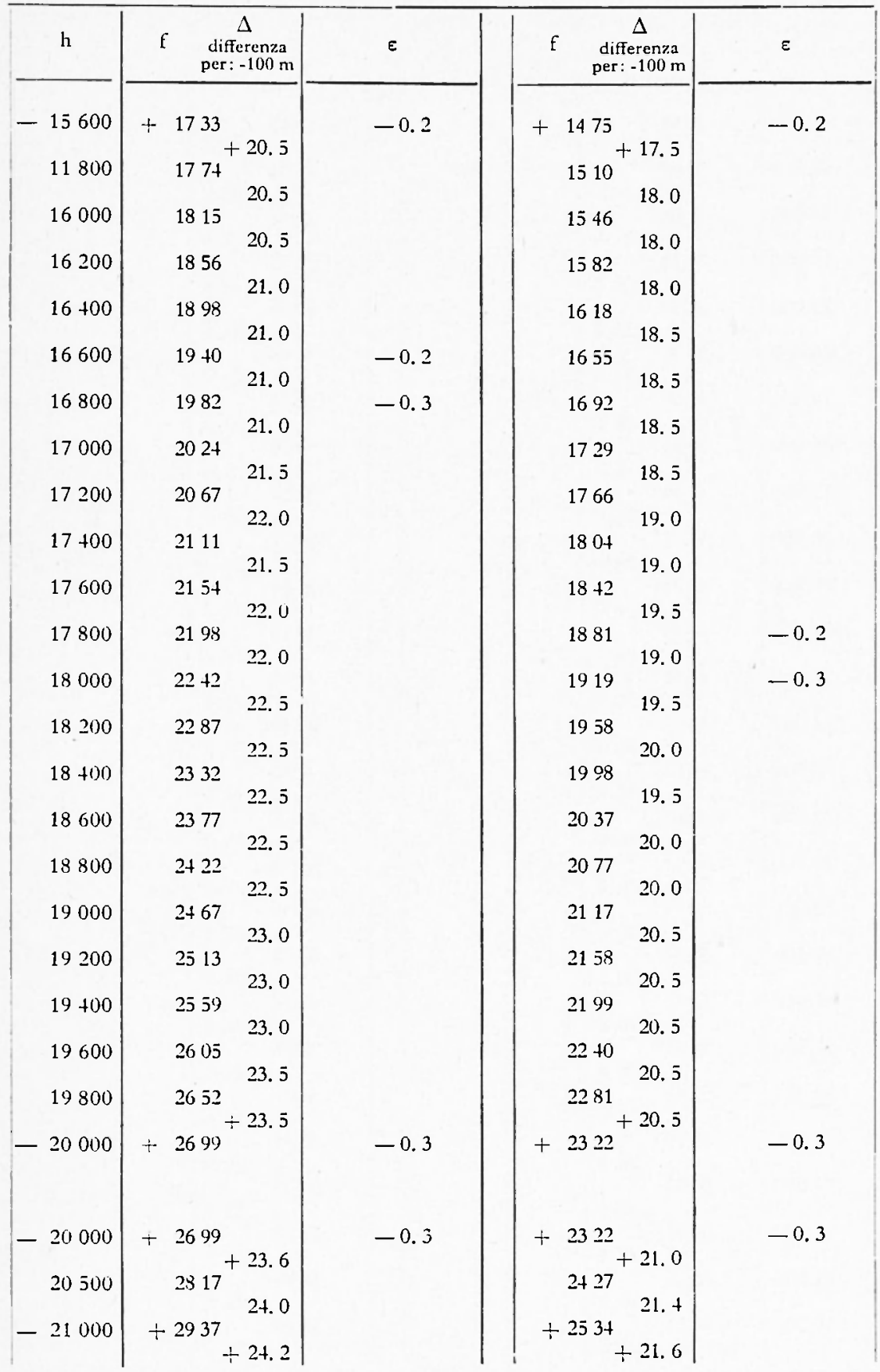


Continus Tal. !'

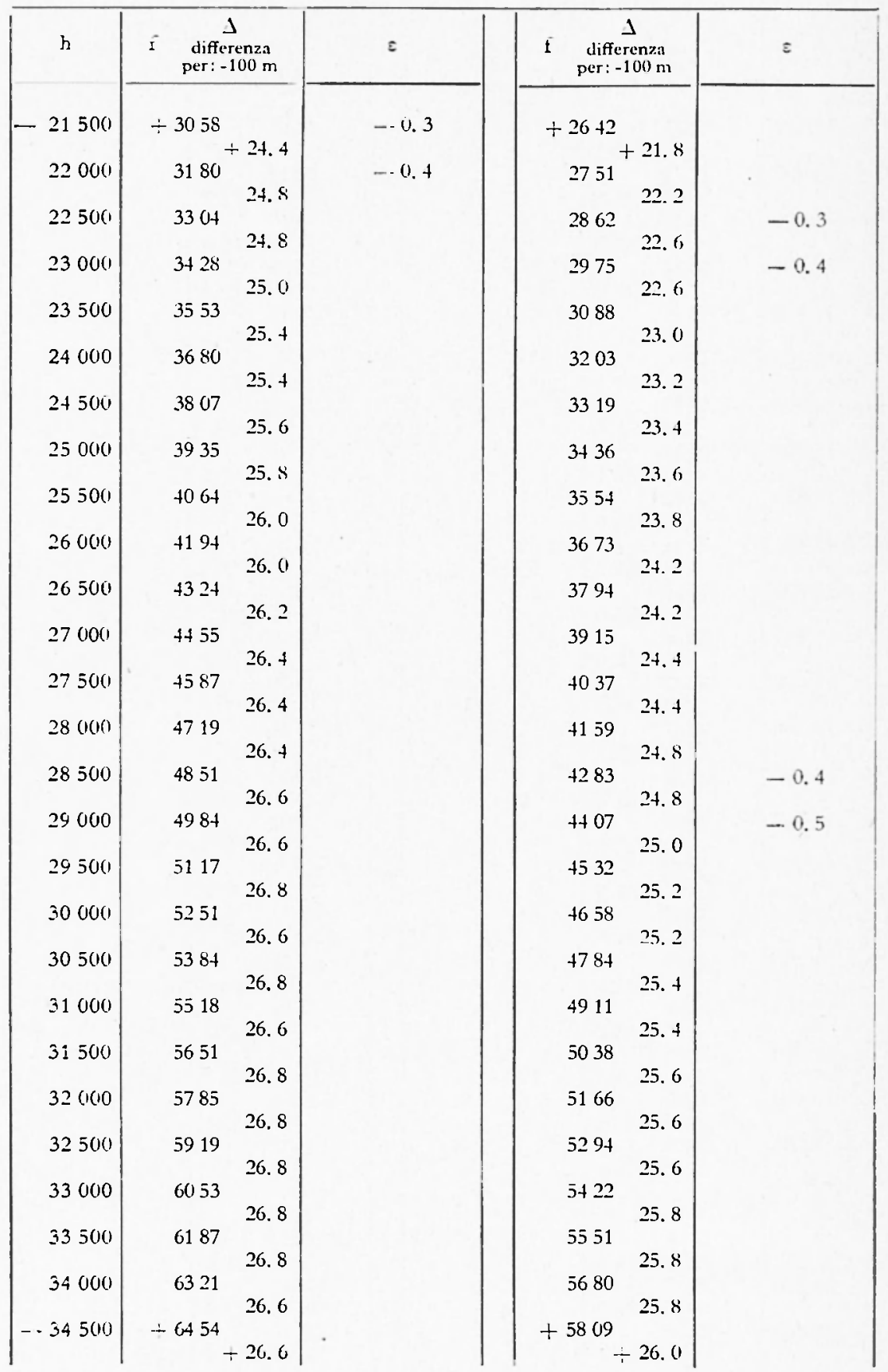


Continua Tab. $V$

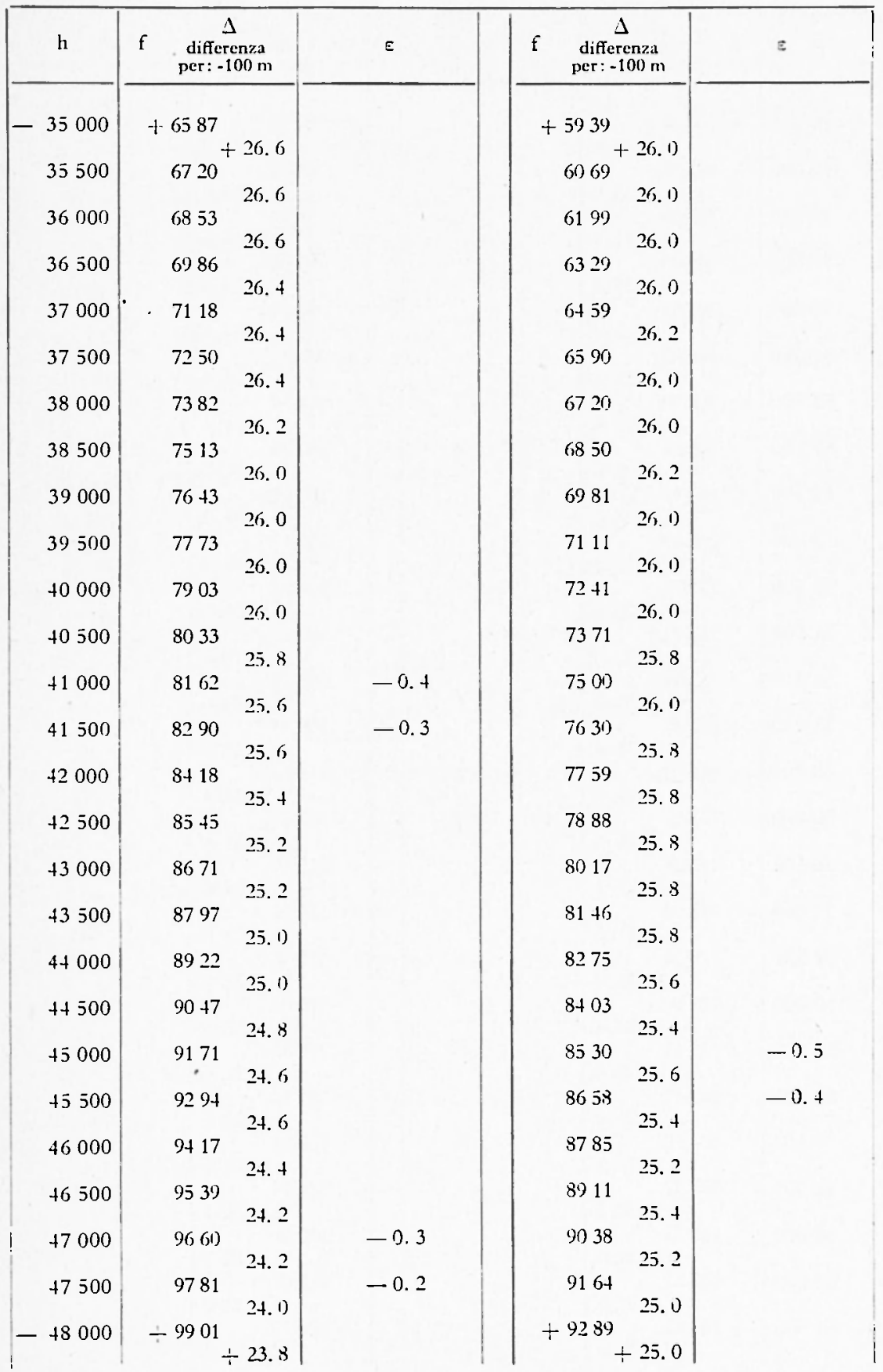


Continua Tah. V

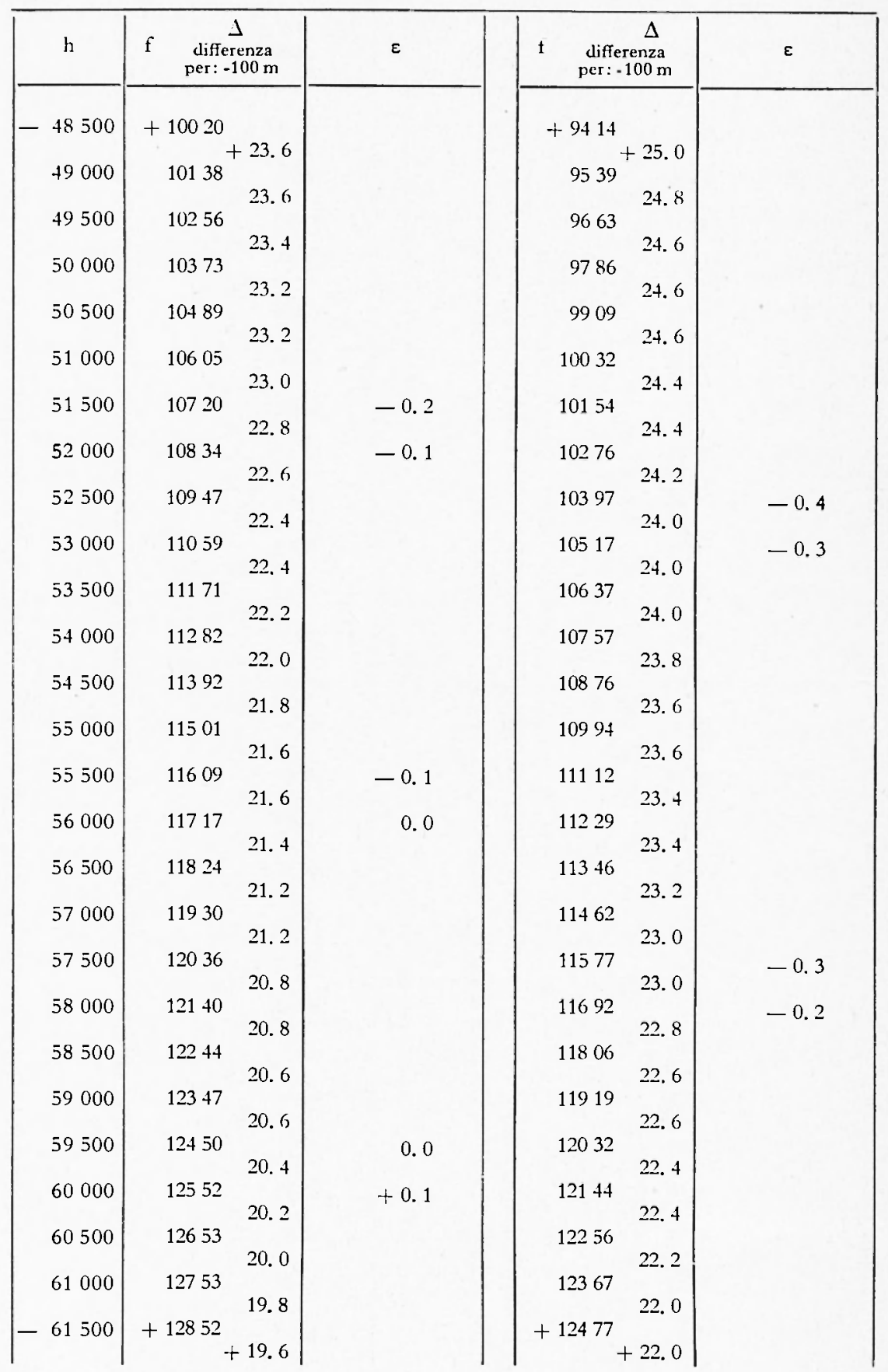


Continua Tab. V

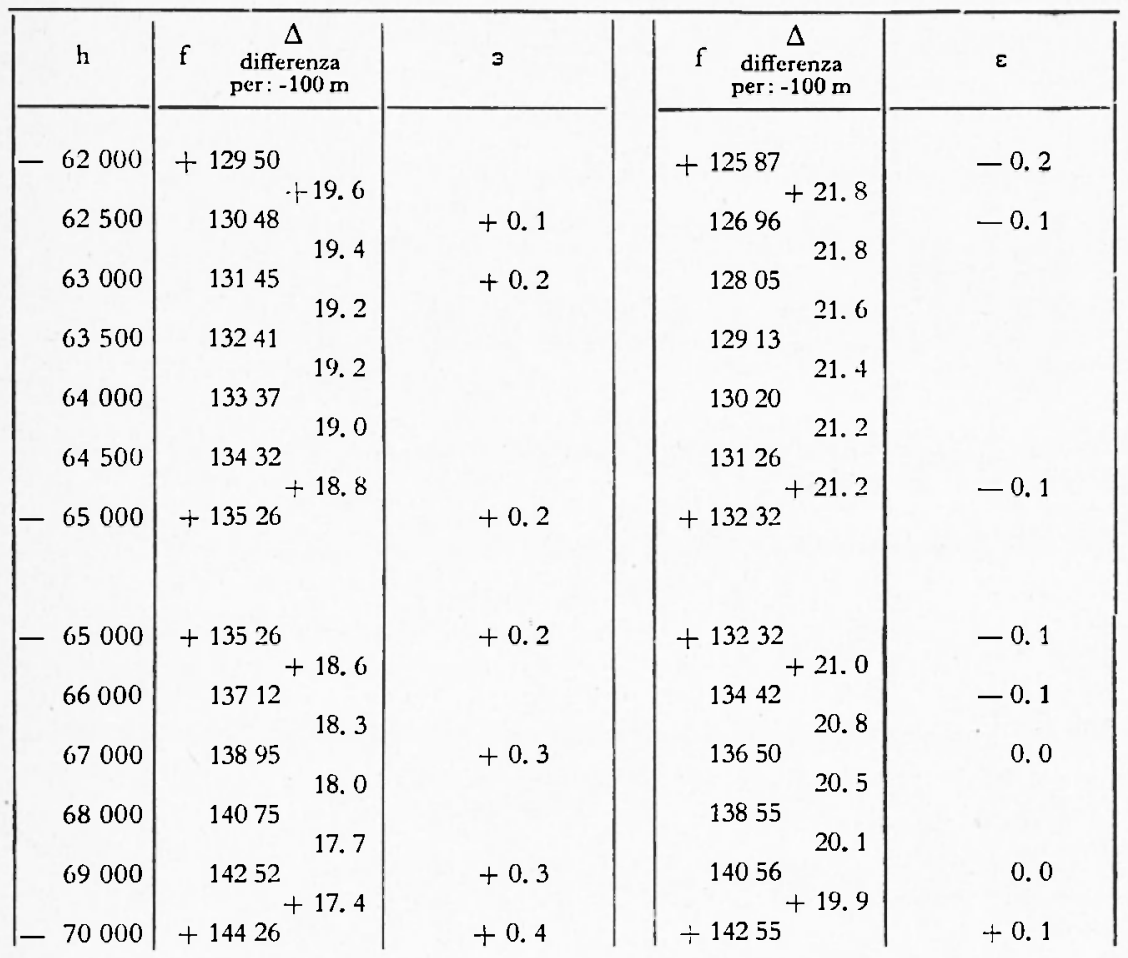




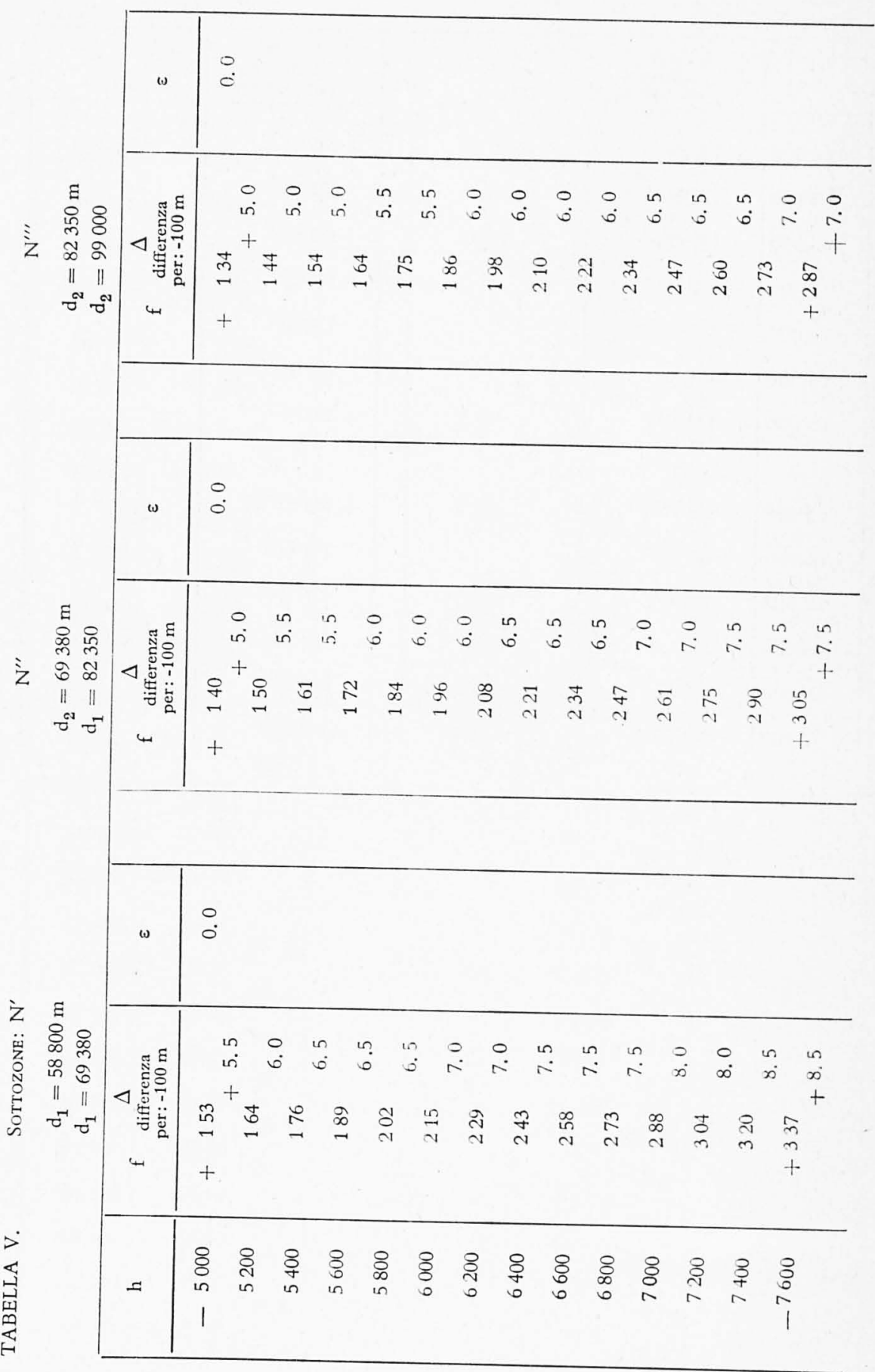




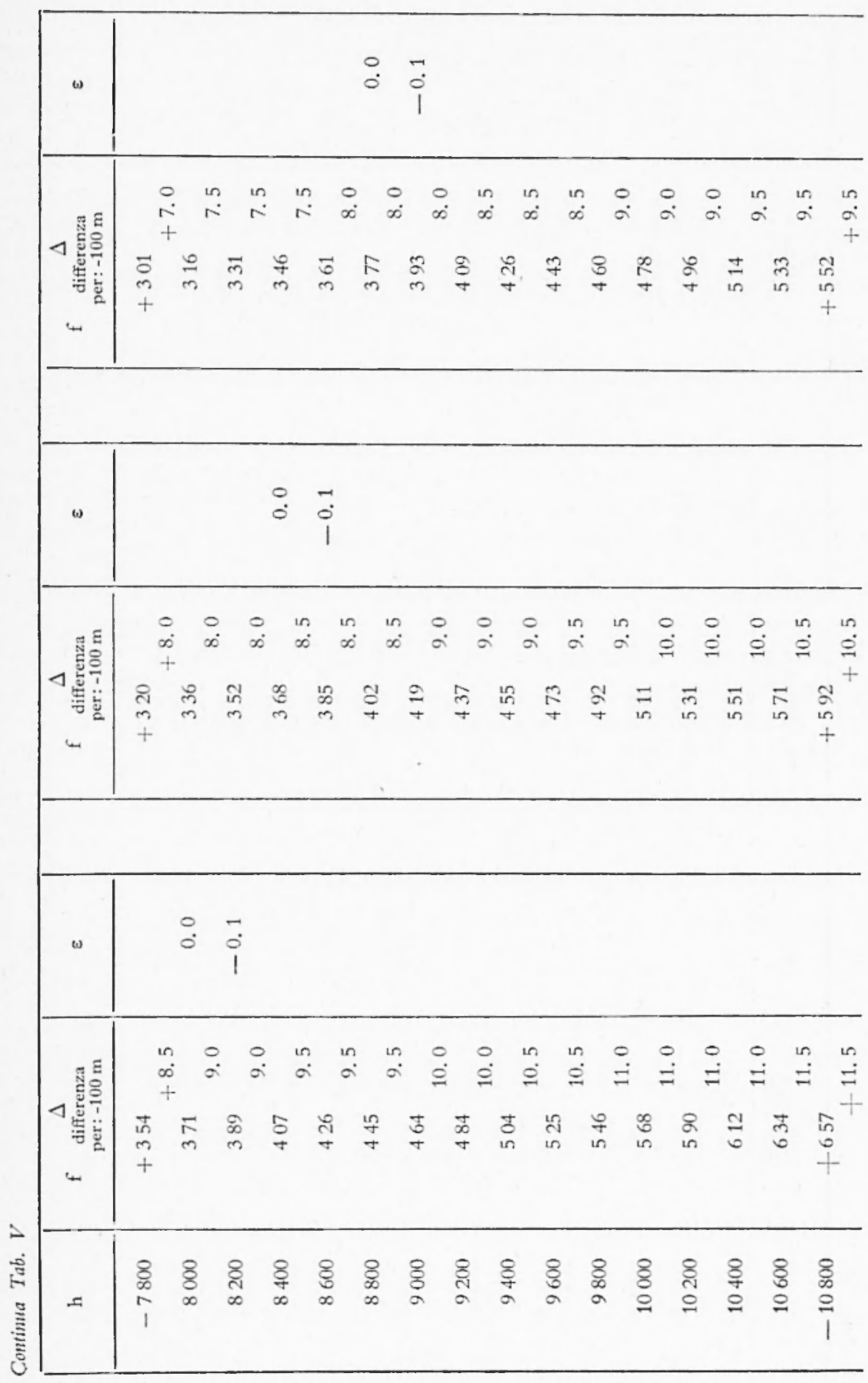




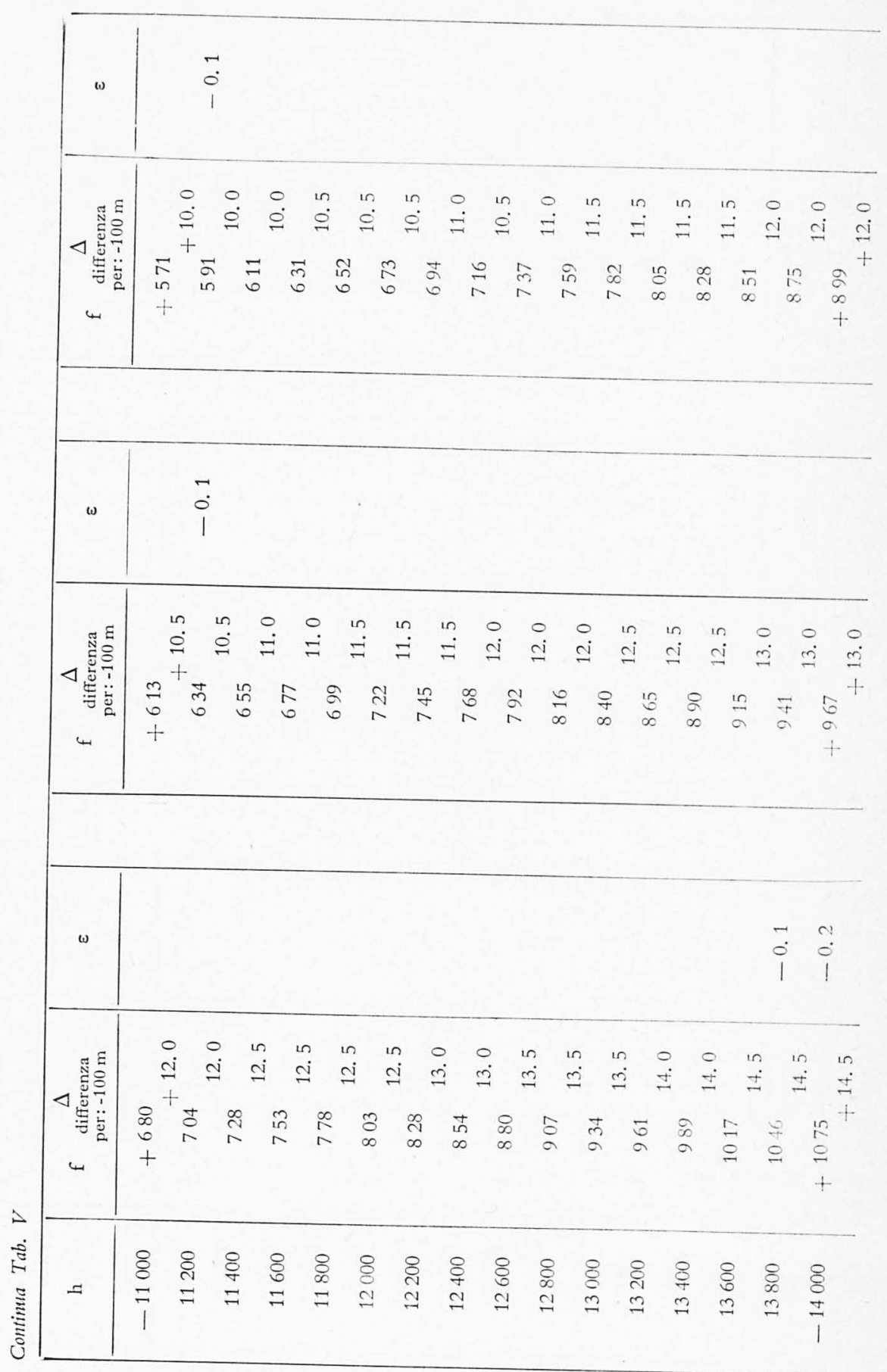




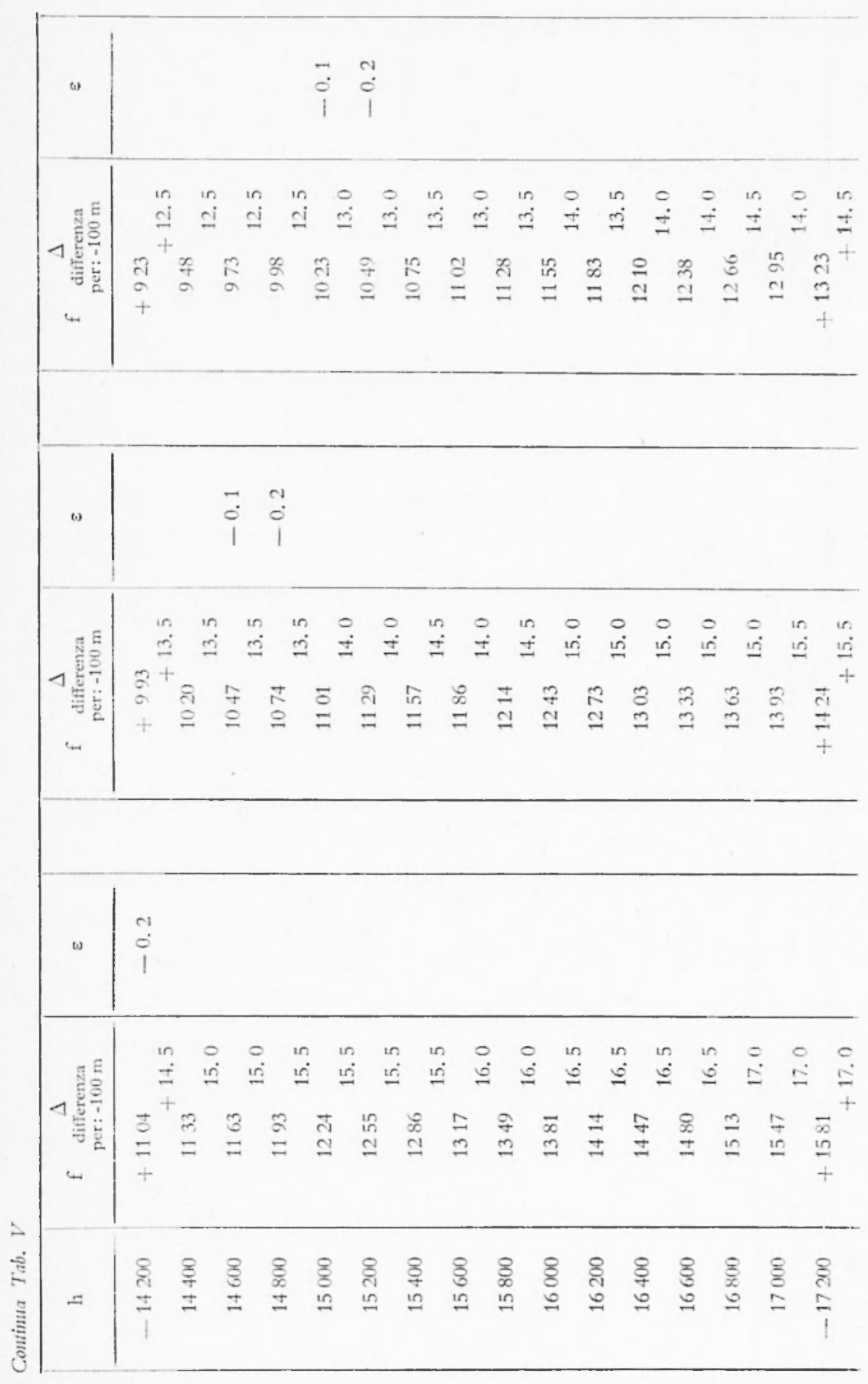




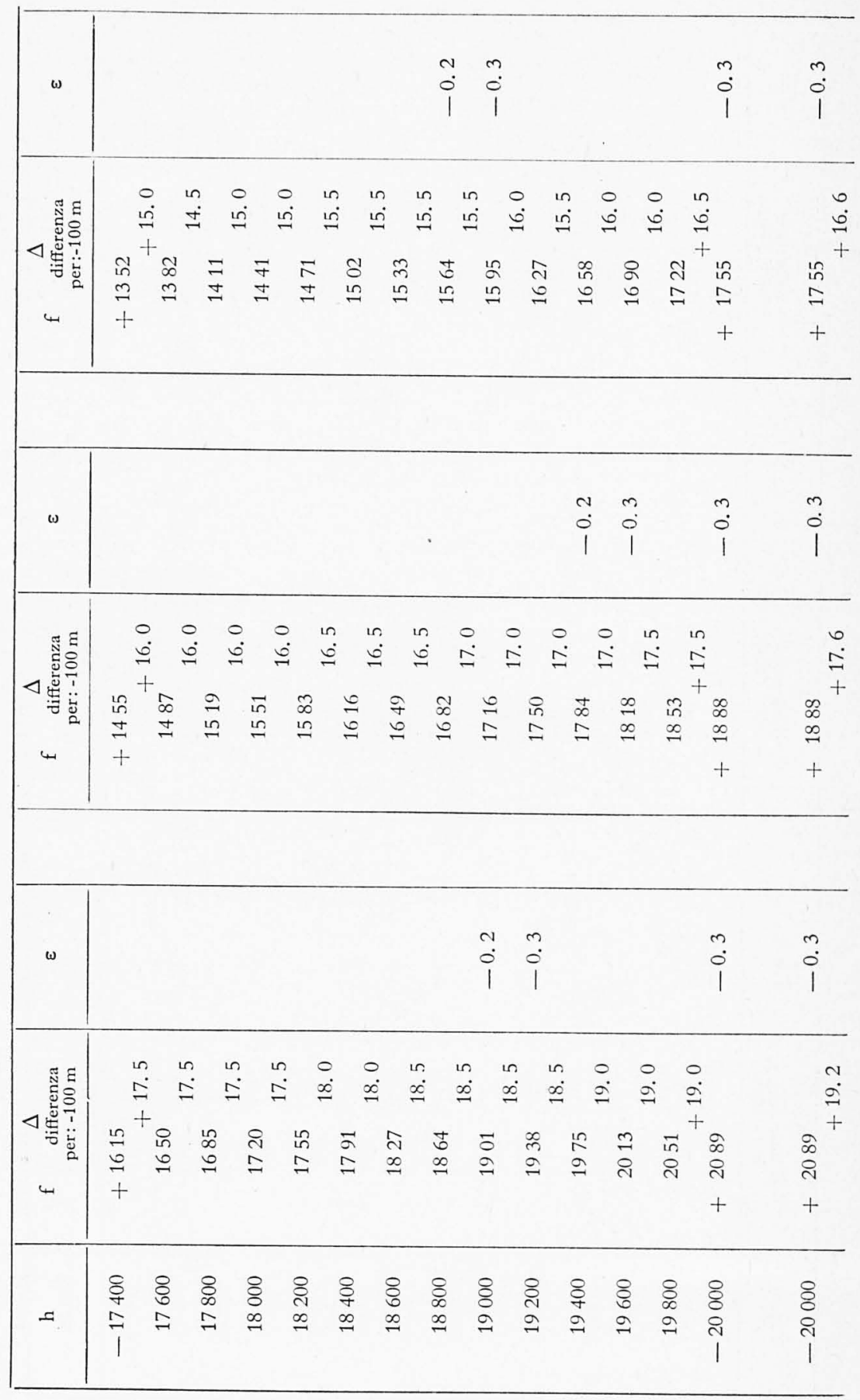




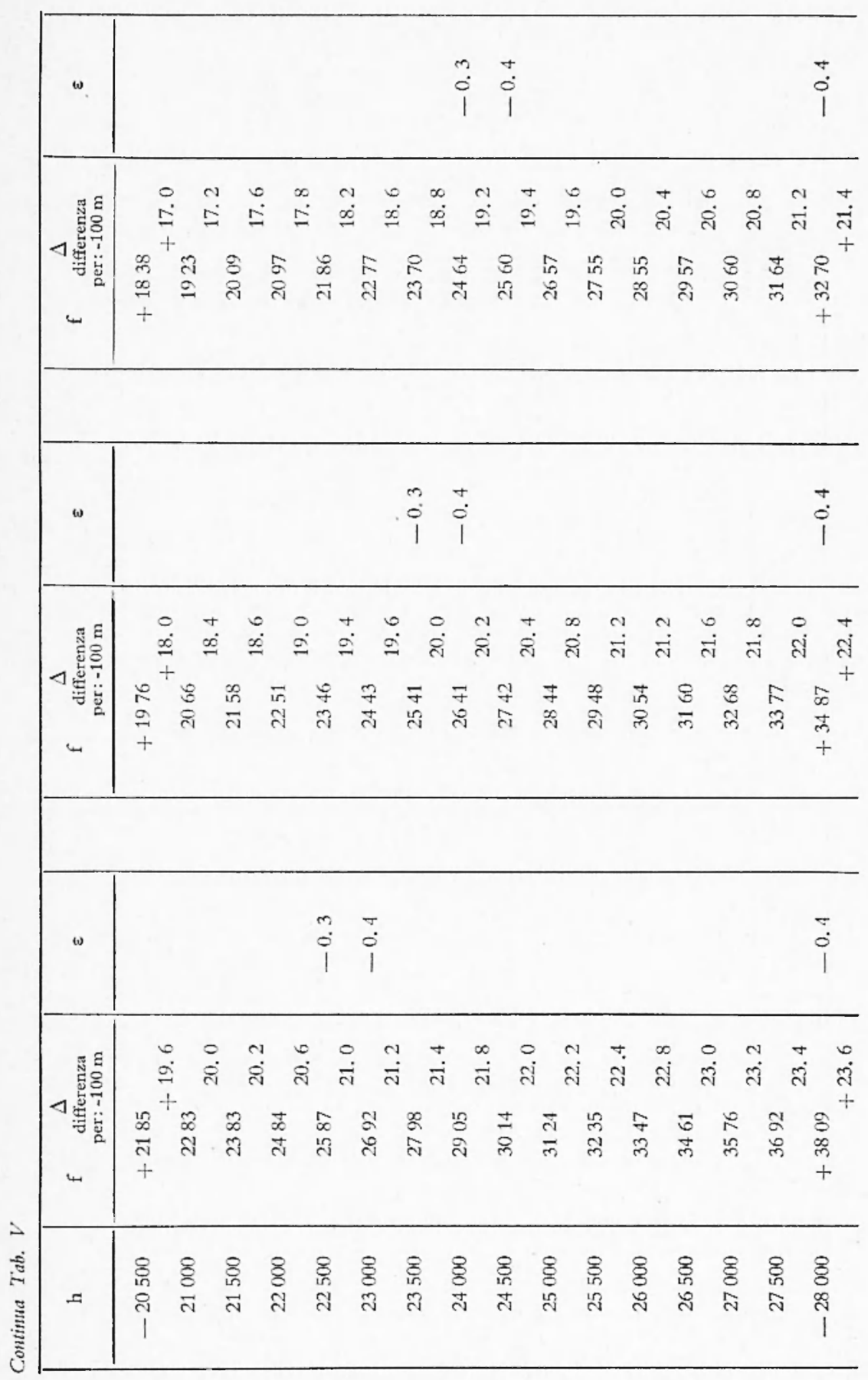




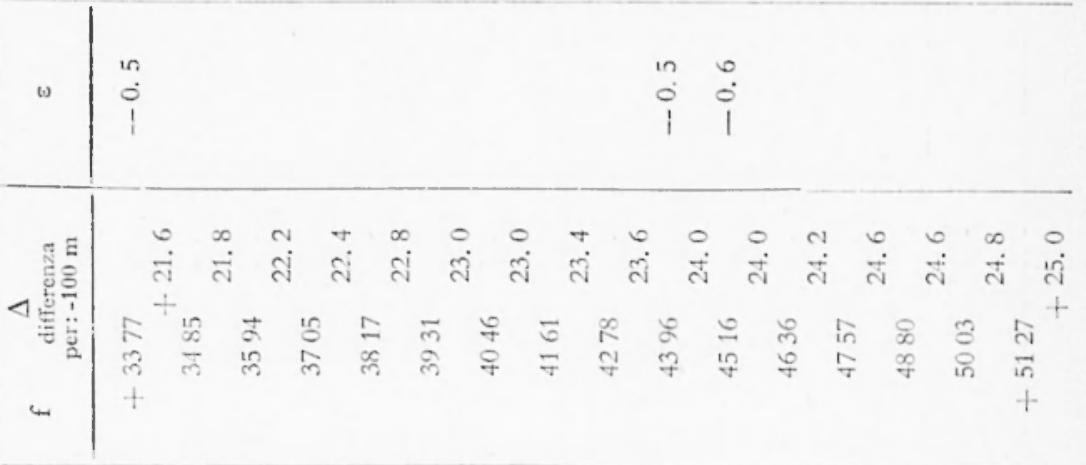

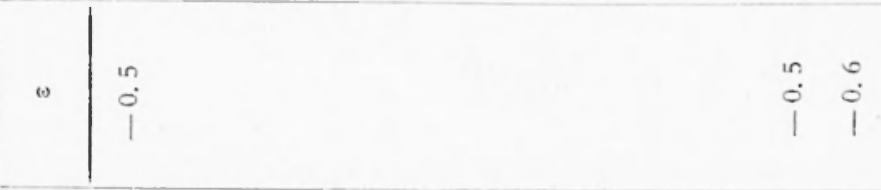

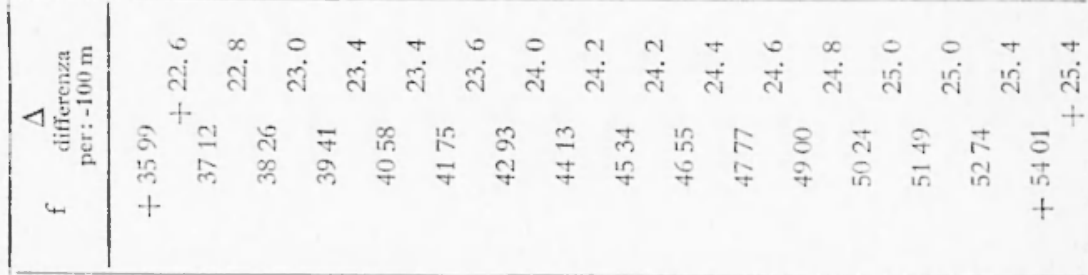

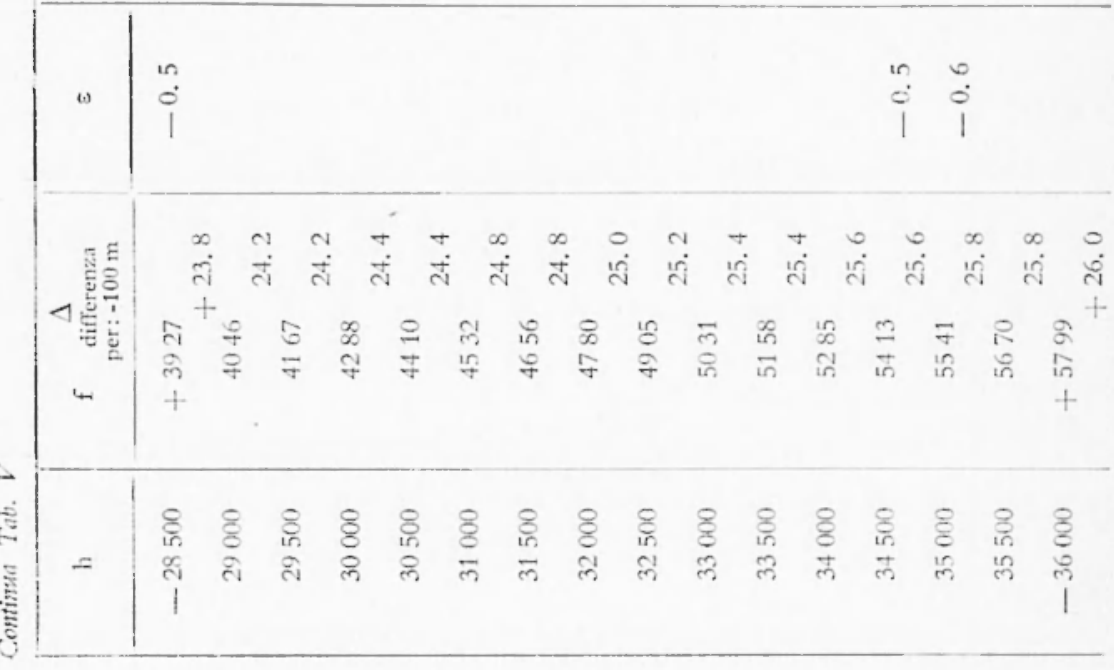




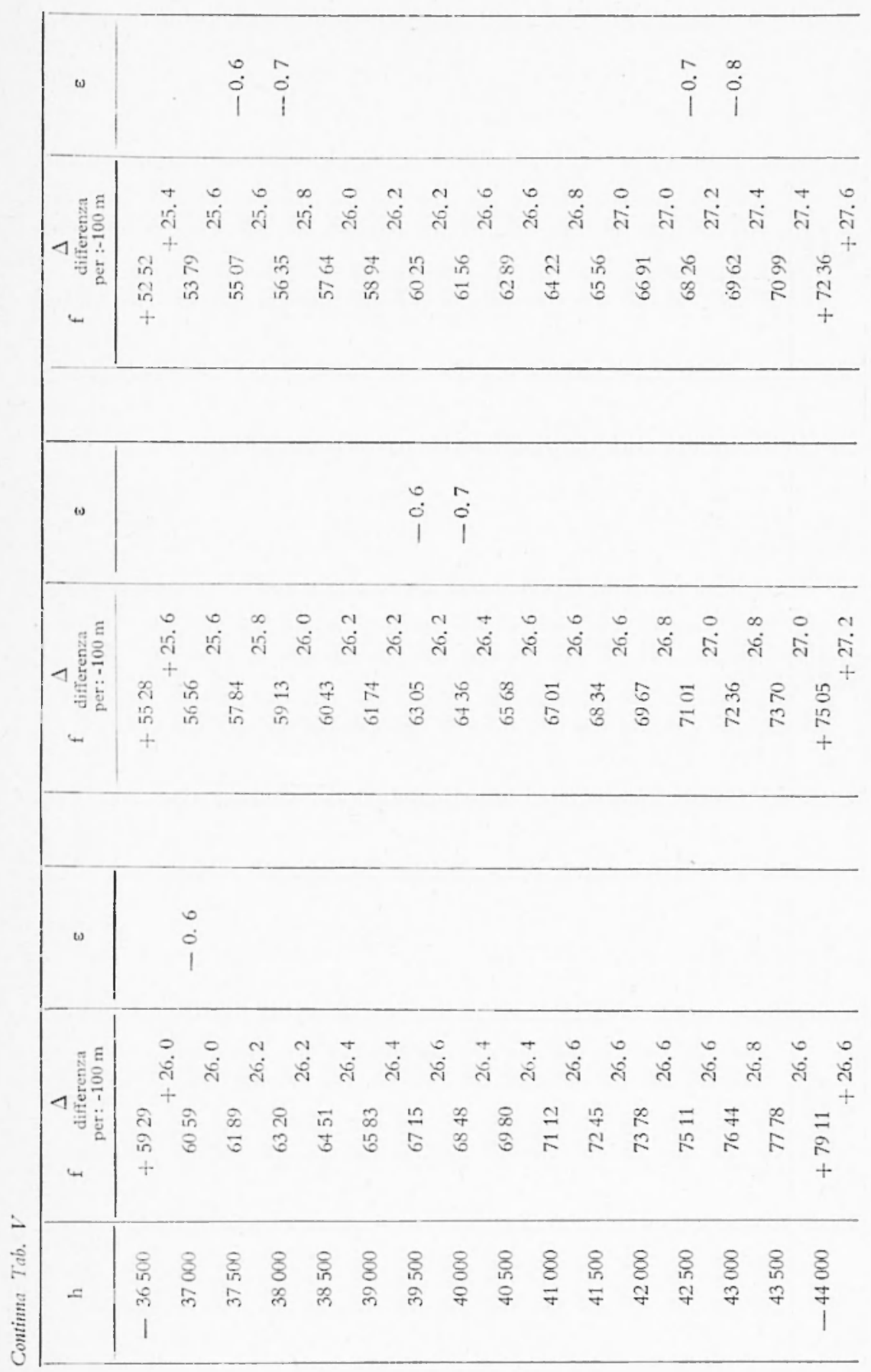




\section{$\stackrel{\infty}{i} i$}

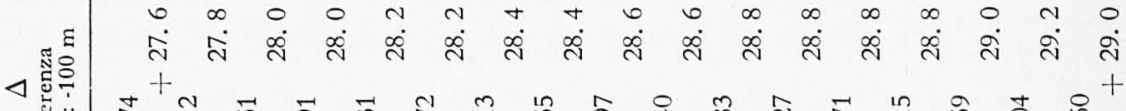

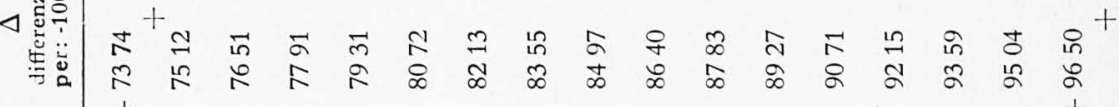

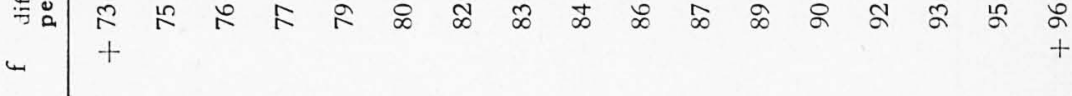

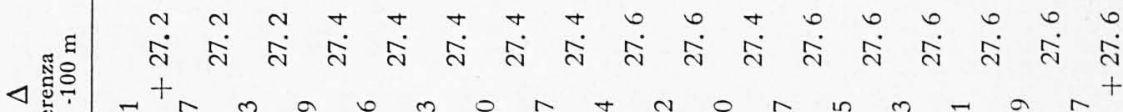

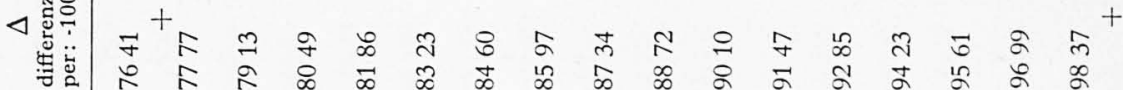

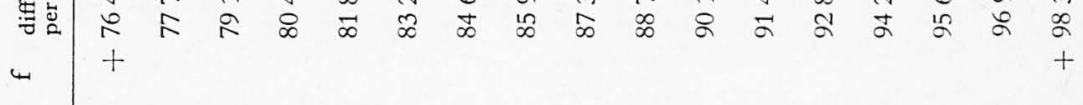
1

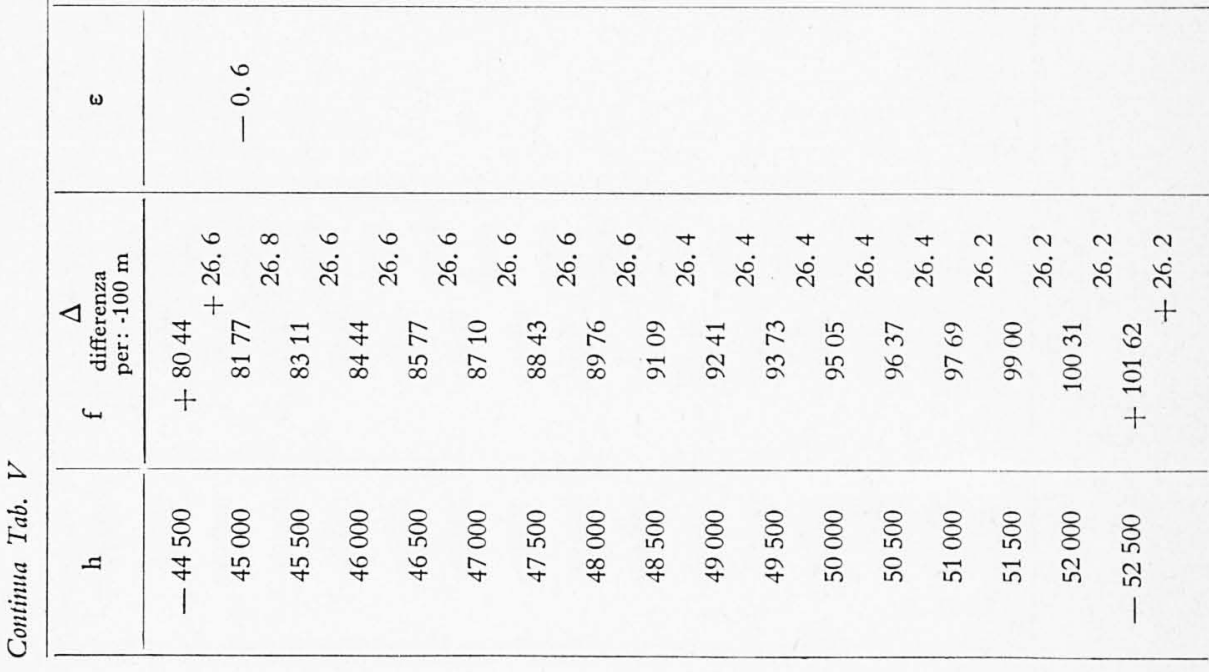




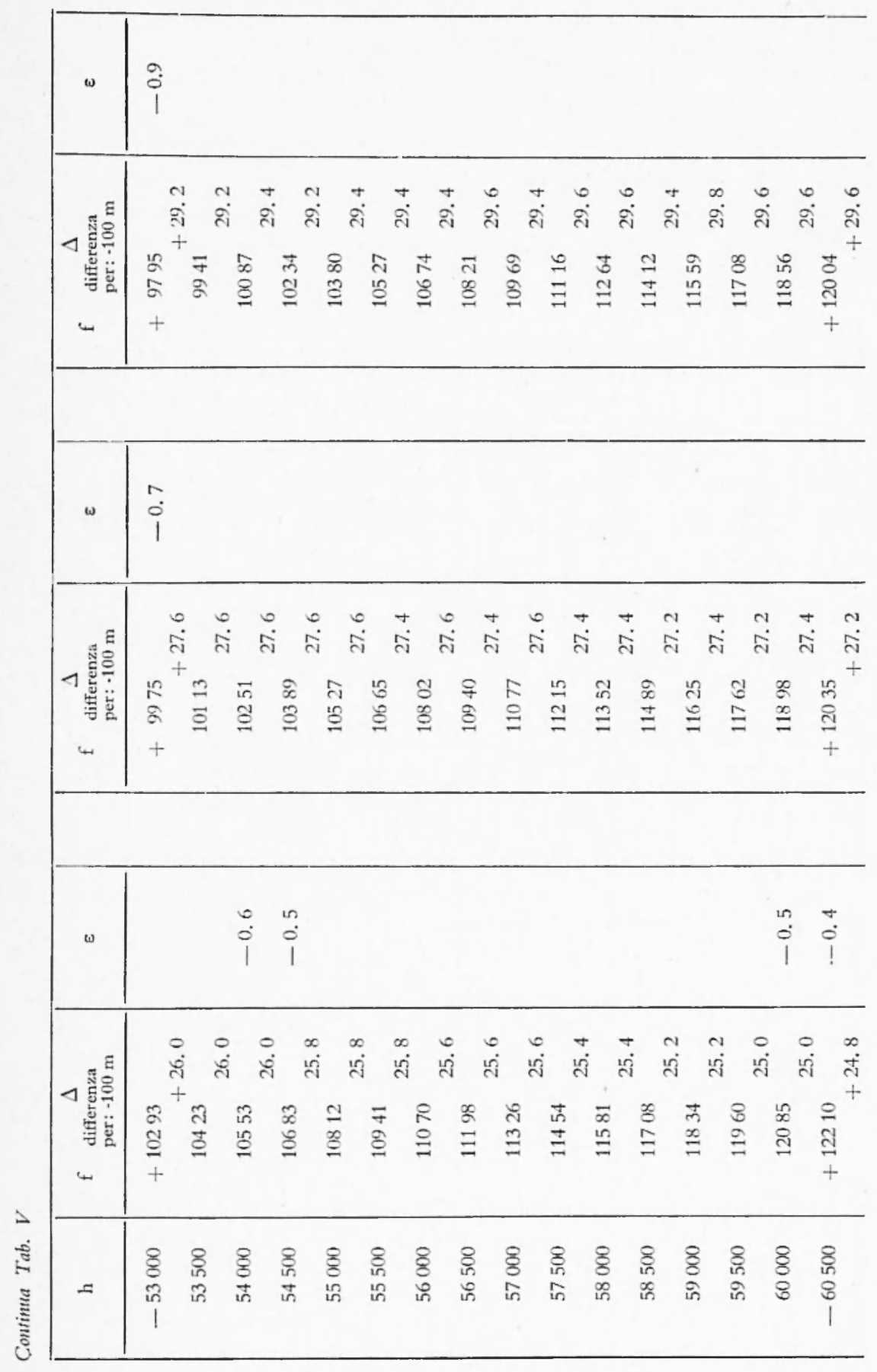




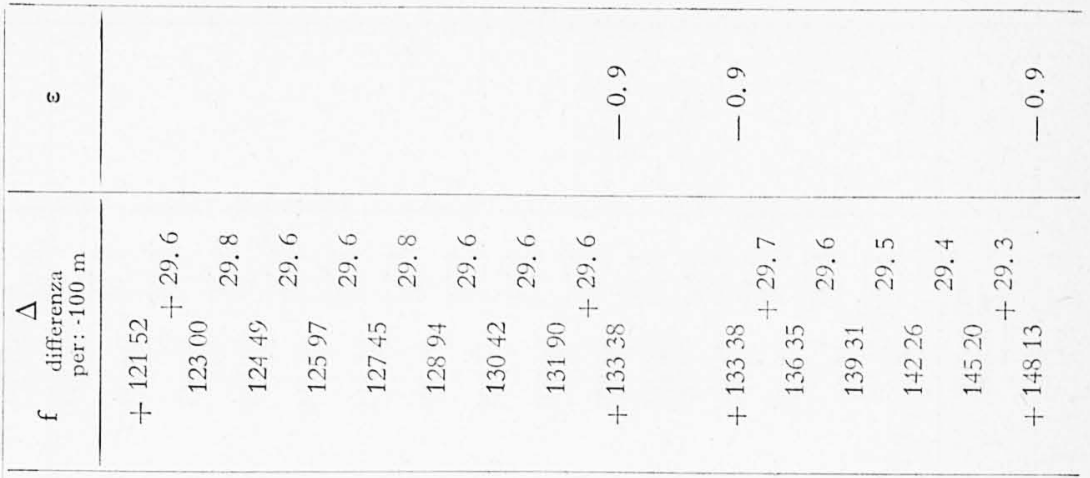

\begin{tabular}{l|l}
\hline \\
\hline
\end{tabular}

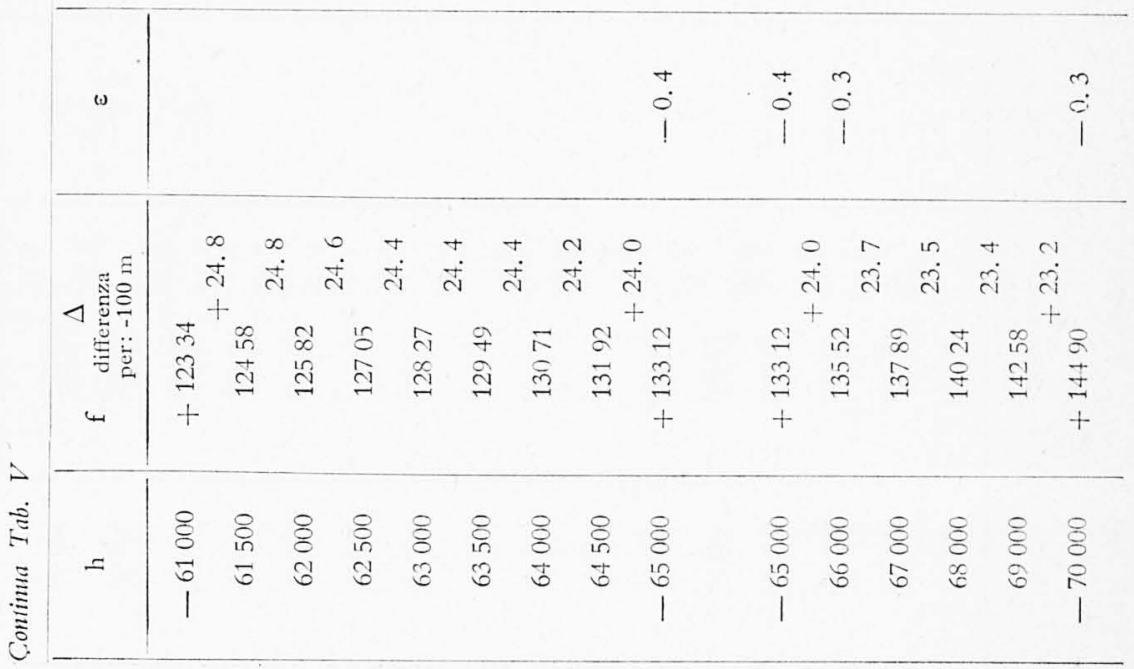


TABTLLA V SOTIOZONE:

$\mathrm{O}_{1}$

$\mathrm{O}^{\prime \prime}{ }_{1}$

$$
\mathrm{d}_{1}=99000 \mathrm{~m}
$$$$
\mathrm{d}_{1}=113900 \mathrm{~m}
$$

$\mathrm{d}_{2}=113900$

$$
\mathrm{d}_{2}=132850
$$

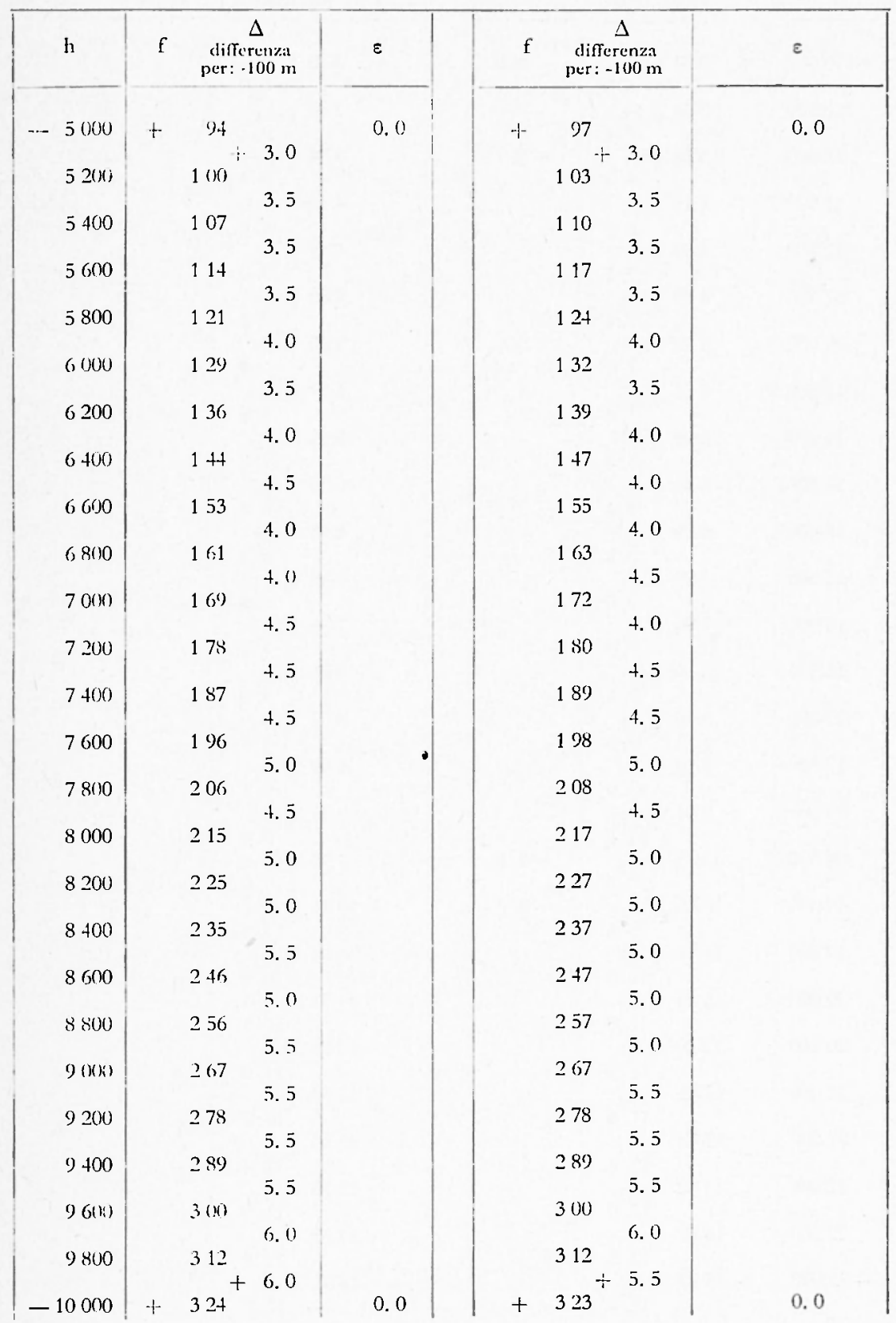


Contima Tab. I

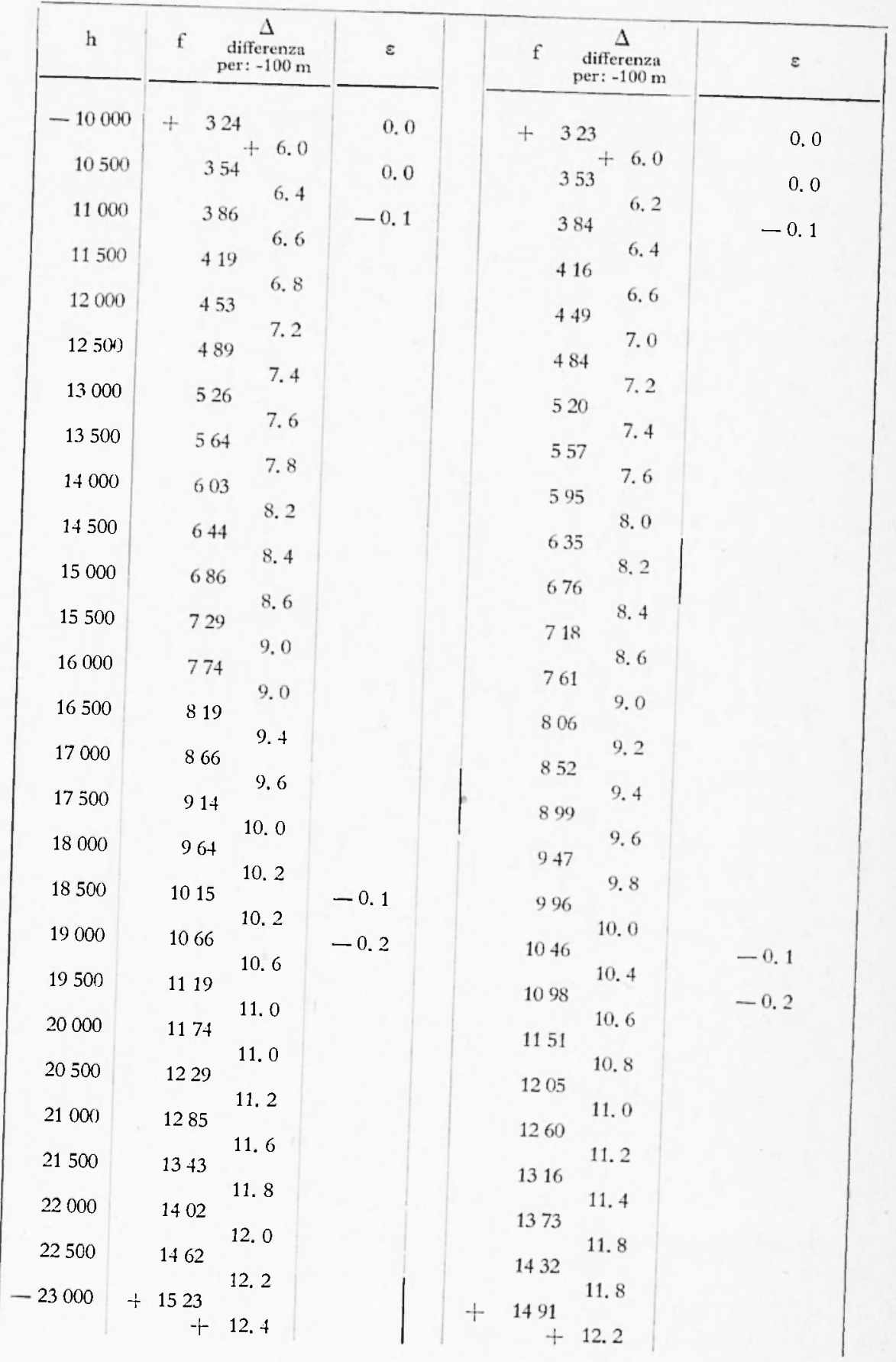


Contimua Tab. I'

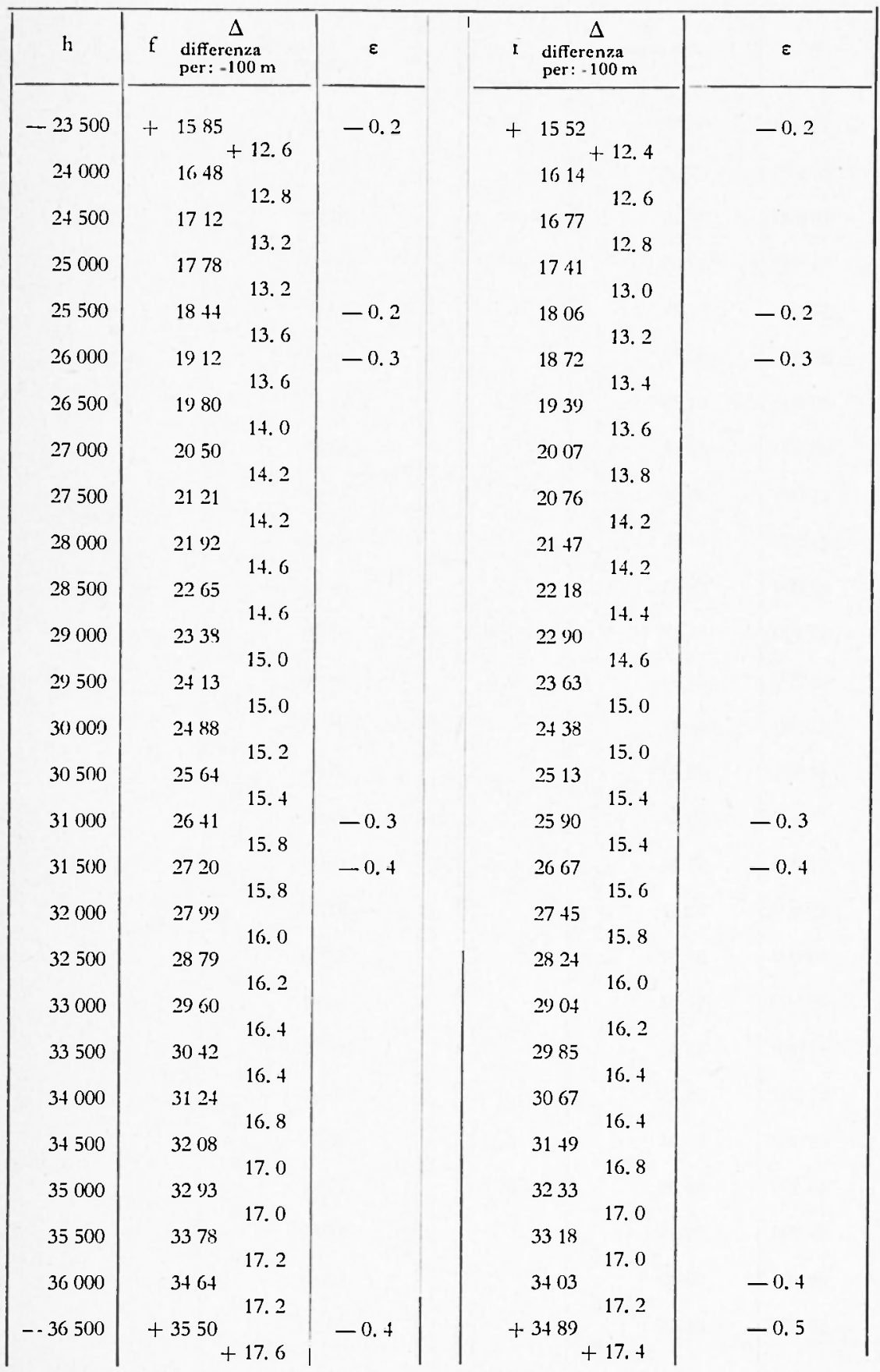


Conimm Tab. Ir

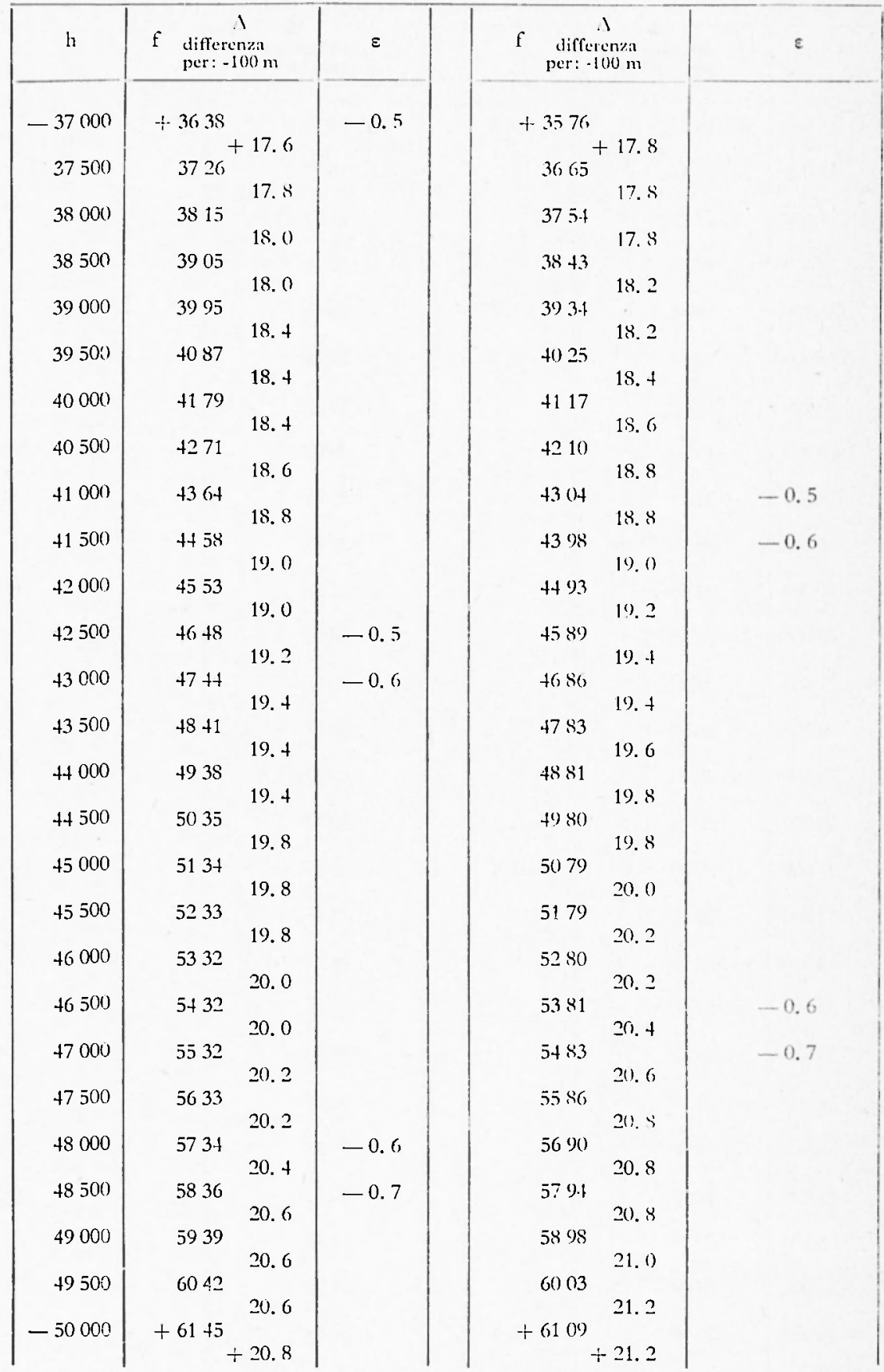


Continna Tiab. 「厂

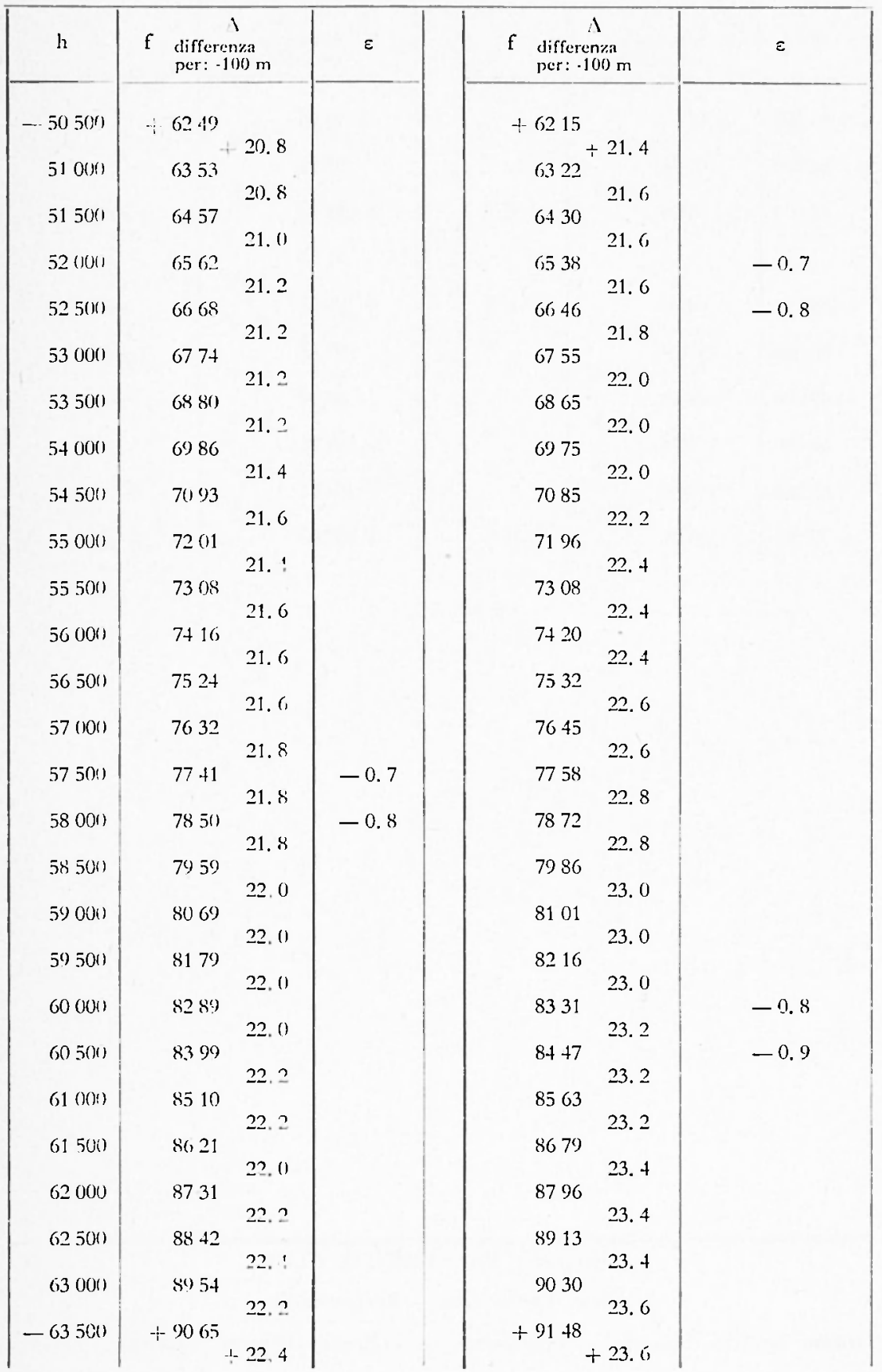


Continua Tab. V

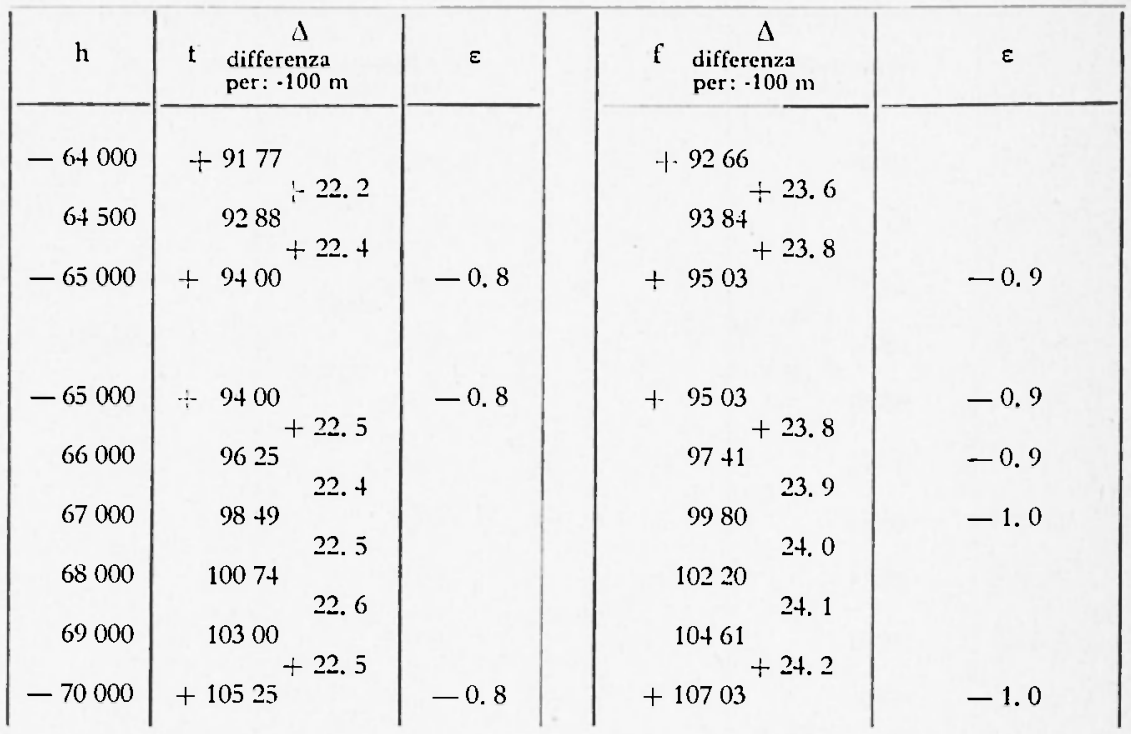

Direltore: Prof. ENRICO MLEDI

Prof. Pietro Caloi - Responsabile

Istituto Grafico Tiberino - Via Gaeta, II - Loma (Officine Grafiche - Tivoli) 